Math. Model. Nat. Phenom.

Vol. 8, No. 1, 2013, pp. 18-47

DOI: $10.1051 / \mathrm{mmnp} / 20138102$

\title{
Properties of Discrete Framelet Transforms
}

\author{
B. Han * \\ Department of Mathematical and Statistical Sciences, University of Alberta \\ Edmonton, Alberta T6G 2G1, Canada
}

\begin{abstract}
As one of the major directions in applied and computational harmonic analysis, the classical theory of wavelets and framelets has been extensively investigated in the function setting, in particular, in the function space $L_{2}\left(\mathbb{R}^{d}\right)$. A discrete wavelet transform is often regarded as a byproduct in wavelet analysis by decomposing and reconstructing functions in $L_{2}\left(\mathbb{R}^{d}\right)$ via nested subspaces of $L_{2}\left(\mathbb{R}^{d}\right)$ in a multiresolution analysis. However, since the input/output data and all filters in a discrete wavelet transform are of discrete nature, to understand better the performance of wavelets and framelets in applications, it is more natural and fundamental to directly study a discrete framelet/wavelet transform and its key properties. The main topic of this paper is to study various properties of a discrete framelet transform purely in the discrete/digital setting without involving the function space $L_{2}\left(\mathbb{R}^{d}\right)$. We shall develop a comprehensive theory of discrete framelets and wavelets using an algorithmic approach by directly studying a discrete framelet transform. The connections between our algorithmic approach and the classical theory of wavelets and framelets in the function setting will be addressed. Using tensor product of univariate complex-valued tight framelets, we shall also present an example of directional tight framelets in this paper.
\end{abstract}

Keywords and phrases: discrete framelet transform, perfect reconstruction, sparsity, stability, dual framelet filter banks, discrete affine systems, linear-phase moments, vanishing moments, sum rules

Mathematics Subject Classification: 42C40, 42C15

\section{Introduction and Motivations}

In this paper we study a discrete framelet/wavelet transform and its various properties. To explain our motivations and to provide necessary background, let us first briefly outline the classical theory of wavelets and framelets in the function space $L_{2}\left(\mathbb{R}^{d}\right)$.

Let $\mathrm{M}$ be a $d \times d$ invertible real-valued matrix. For any square integrable function $f \in L_{2}\left(\mathbb{R}^{d}\right)$, throughout the paper we shall adopt the following notation:

$$
f_{\mathrm{M} ; \mathrm{k}, \mathrm{n}}(x):=\llbracket \mathrm{M} ; \mathrm{k}, \mathrm{n} \rrbracket f(x):=|\operatorname{det}(\mathrm{M})|^{1 / 2} e^{-i \mathrm{n} \cdot \mathrm{M} x} f(\mathrm{M} x-\mathrm{k}), \quad x, \mathrm{k}, \mathrm{n} \in \mathbb{R}^{d},
$$

where $i$ denotes the imaginary unit. In particular, we define $f_{\mathrm{M} ; \mathrm{k}}:=f_{\mathrm{M} ; \mathrm{k}, \mathbf{0}}$. Let $\Psi$ be a (finite) subset of $L_{2}\left(\mathbb{R}^{d}\right)$. The following homogeneous M-affine (or M-wavelet) system

${ }^{*}$ Corresponding author. E-mail: bhan@ualberta.ca 


$$
\operatorname{AS}(\Psi):=\left\{\psi_{\mathrm{M}^{j} ; \mathrm{k}} \quad: \quad j \in \mathbb{Z}, \mathrm{k} \in \mathbb{Z}^{d}, \psi \in \Psi\right\},
$$

has been extensively studied in the function space $L_{2}\left(\mathbb{R}^{d}\right)$ in the literature of wavelet analysis, often with $M$ being an expansive integer matrix. Here we say that $M$ is expansive if all its eigenvalues have modulus greater than one. Elements in $\Psi$ are often called wavelet (generating) functions. To emphasize the dilation matrix $\mathrm{M}$ in an affine system, we also use $\operatorname{AS}^{\mathrm{M}}(\Psi)$ instead of $\operatorname{AS}(\Psi)$.

There are several different types of wavelets and framelets. The affine system $\operatorname{AS}(\Psi)$ could be an orthonormal basis, a tight frame, or a Riesz basis for the function space $L_{2}\left(\mathbb{R}^{d}\right)$. For example, we say that $\operatorname{AS}(\Psi)$ is a tight frame for $L_{2}\left(\mathbb{R}^{d}\right)$ if

$$
\|f\|_{L_{2}\left(\mathbb{R}^{d}\right)}^{2}=\sum_{j \in \mathbb{Z}} \sum_{\mathbf{k} \in \mathbb{Z}^{d}} \sum_{\psi \in \Psi}\left|\left\langle f, \psi_{\mathrm{M}^{j} ; \mathrm{k}}\right\rangle\right|^{2}, \quad \forall f \in L_{2}\left(\mathbb{R}^{d}\right) .
$$

It follows from (1.3) that every function $f \in L_{2}\left(\mathbb{R}^{d}\right)$ has the following wavelet representation:

$$
f=\sum_{j \in \mathbb{Z}} \sum_{\mathbf{k} \in \mathbb{Z}^{d}} \sum_{\psi \in \Psi}\left\langle f, \psi_{\mathrm{M}^{j} ; \mathrm{k}}\right\rangle \psi_{\mathrm{M}^{j} ; \mathrm{k}}
$$

with the series converging in $L_{2}\left(\mathbb{R}^{d}\right)$.

One of the major tasks in wavelet analysis is to construct $\Psi$ having some desirable properties so that $\mathrm{AS}(\Psi)$ is an orthonormal basis, a tight frame, or a Riesz basis for $L_{2}\left(\mathbb{R}^{d}\right)$. For this purpose, the dominating approach in the current literature is to use a multiresolution analysis (MRA). A sequence $\left\{V_{j}\right\}_{j \in \mathbb{Z}}$ of closed subspaces of $L_{2}\left(\mathbb{R}^{d}\right)$ is called a multiresolution analysis of $L_{2}\left(\mathbb{R}^{d}\right)($ see $[2,8,35])$ if

(i) $V_{j} \subseteq V_{j+1}$ and $V_{j}=\left\{f\left(\mathrm{M}^{j} \cdot\right): f \in V_{0}\right\}$ for all $j \in \mathbb{Z}$;

(ii) $\cup_{j \in \mathbb{Z}} V_{j}$ is dense in $L_{2}\left(\mathbb{R}^{d}\right)$ and $\cap_{j \in \mathbb{Z}} V_{j}=\{0\}$;

(iii) There exists $\Phi \subseteq L_{2}\left(\mathbb{R}^{d}\right)$ such that the linear span of $\phi(\cdot-\mathrm{k}), \mathrm{k} \in \mathbb{Z}^{d}, \phi \in \Phi$ is dense in $V_{0}$.

$\left\{\phi(\cdot-\mathrm{k}): \mathrm{k} \in \mathbb{Z}^{d}, \phi \in \Phi\right\}$ is often required to be a Riesz basis or an orthonormal basis of $V_{0}$. By (i), we have $\Phi \subseteq V_{1}$ and therefore, $\Phi$ must be M-refinable satisfying $\widehat{\phi}\left(\mathrm{M}^{\top} \xi\right)=\widehat{a}(\xi) \widehat{\phi}(\xi)$ for almost every $\xi \in \mathbb{R}^{d}$, where $\phi$ is obtained by listing all the elements in $\Phi$ as a column vector and $\widehat{a}$ is a $(\# \Phi) \times(\# \Phi)$ matrix of $2 \pi \mathbb{Z}^{d}$-periodic Lebesgue measurable functions. Now a set $\Psi$ of wavelet generating functions is often derived from $\phi$ via the following relation:

$$
\widehat{\psi}\left(\mathrm{M}^{\top} \xi\right)=\widehat{b_{\psi}}(\xi) \widehat{\phi}(\xi), \quad \text { a.e. } \xi \in \mathbb{R}^{d}, \psi \in \Psi,
$$

where $\widehat{b_{\psi}}$ is some $1 \times(\# \Phi)$ row vector of $2 \pi \mathbb{Z}^{d}$-periodic measurable functions. By appropriately constructing $\widehat{a}$ and $\widehat{b_{\psi}}, \psi \in \Psi$, one obtains an affine system $\operatorname{AS}(\Psi)$ so that it is an orthonormal basis, a tight frame, or a Riesz basis for $L_{2}\left(\mathbb{R}^{d}\right)$. There are a vast literature on this topic, to mention a very tiny portion of them, see $[2,8,35,36,39,40]$ and numerous references therein.

We now discuss the associated discrete wavelet/framelet transform in the function setting by decomposing and reconstructing functions in $L_{2}\left(\mathbb{R}^{d}\right)$ via nested subspaces of $L_{2}\left(\mathbb{R}^{d}\right)$ in a multiresolution analysis. For simplicity of discussion, we assume that $\Phi=\{\phi\}$ is a singleton and $\operatorname{AS}(\Psi)$ is a tight frame for $L_{2}\left(\mathbb{R}^{d}\right)$ with $\Psi$ being derived from an M-refinable function $\phi$ via (1.5). To compute the wavelet coefficients $\left\langle f, \psi_{\mathrm{M}^{j} ; \mathrm{k}}\right\rangle$ in the wavelet representation of $f$ in (1.4), a widely accepted approach in the literature consists of two parts: projection (or discretization) and a discrete transform. To discretize a function $f$ from the continuum domain into the discrete/digital domain, one selects a large enough integer $J$ so that the function $f$ is well approximated by the projected function $\mathcal{P}_{J} f:=\sum_{\mathrm{k} \in \mathbb{Z}^{d}}\left(\mathbb{D}_{J} f\right)(\mathrm{k}) \phi_{\mathrm{M}^{J} ; \mathrm{k}}$, where $\mathbb{D}_{j}, j \in \mathbb{Z}$ are the discretization (or approximation) operators defined by

$$
\mathbb{D}_{j}: L_{2}\left(\mathbb{R}^{d}\right) \rightarrow l_{2}\left(\mathbb{Z}^{d}\right) \quad \text { with } \quad \mathbb{D}_{j} f:=\left\{\left\langle f, \phi_{\mathrm{M}^{j} ; \mathrm{k}}\right\rangle\right\}_{\mathrm{k} \in \mathbb{Z}^{d}} .
$$


Therefore, most information of the function $f$ is encoded now in the discrete sequence $\mathbb{D}_{J} f$. Next a discrete transform is employed to compute all wavelet coefficients $\left\langle f, \psi_{\mathrm{M}^{j} ; \mathrm{k}}\right\rangle, j<J$ and $\mathrm{k} \in \mathbb{Z}^{d}$ using only the discrete sequence $\mathbb{D}_{J} f$ and the Fourier coefficients of $\widehat{a}$ and $\widehat{b_{\psi}}, \psi \in \Psi$. More precisely, rewrite the relations $\widehat{\phi}\left(\mathrm{M}^{\top} \xi\right)=\widehat{a}(\xi) \widehat{\phi}(\xi)$ and $\widehat{\psi}\left(\mathrm{M}^{\top} \xi\right)=\widehat{b}(\xi) \widehat{\phi}(\xi)$ as

$$
\phi(x)=\mathrm{d}_{\mathrm{M}} \sum_{\mathbf{k} \in \mathbb{Z}^{d}} a(\mathrm{k}) \phi(\mathrm{M} x-\mathrm{k}) \quad \text { and } \quad \psi(x)=\mathrm{d}_{\mathrm{M}} \sum_{\mathbf{k} \in \mathbb{Z}^{d}} b(\mathrm{k}) \phi(\mathrm{M} x-\mathrm{k}),
$$

where $\mathrm{d}_{\mathrm{M}}:=|\operatorname{det}(\mathrm{M})|$ and $\sum_{\mathrm{k} \in \mathbb{Z}^{d}} a(\mathrm{k}) e^{-i \mathrm{k} \cdot \xi}:=\widehat{a}(\xi)$, that is, $\{a(\mathrm{k})\}_{\mathrm{k} \in \mathbb{Z}^{d}}$ is the sequence of the Fourier coefficients of $\widehat{a}$. Then one can easily deduce that

$$
\left(\mathbb{D}_{j-1} f\right)(\mathrm{n})=\mathrm{d}_{\mathrm{M}}^{1 / 2} \sum_{\mathrm{k} \in \mathbb{Z}^{d}} \overline{a(\mathrm{k}-\mathrm{Mn})}\left(\mathbb{D}_{j} f\right)(\mathrm{k}), \quad\left\langle f, \psi_{\mathrm{M}^{j-1} ; \mathrm{n}}\right\rangle=\mathrm{d}_{\mathrm{M}}^{1 / 2} \sum_{\mathrm{k} \in \mathbb{Z}^{d}} \overline{b(\mathrm{k}-\mathrm{Mn})}\left(\mathbb{D}_{j} f\right)(\mathrm{k}) .
$$

Now one can easily see that all the coefficients $\left\langle f, \psi_{\mathrm{M}^{j} ; \mathrm{n}}\right\rangle, j<J$ and $\mathrm{n} \in \mathbb{Z}^{d}$ can be computed recursively using $\mathbb{D}_{J} f$ and the Fourier coefficients of $\widehat{a}$ and $\widehat{b_{\psi}}, \psi \in \Psi$. This discrete transform is called a fast wavelet transform in the literature (see Sections 2 and 4 for more detail).

However, the mapping $\mathbb{D}_{J}$ in (1.6) may fail to be onto and therefore, not all signals in $l_{2}\left(\mathbb{Z}^{d}\right)$ can be exactly reconstructed by the associated discrete wavelet transform. This is not desirable in both theory and application, since $\left\langle f, \phi_{\mathrm{M}^{J} ; \mathrm{k}}\right\rangle$ is often numerically computed by quadrature formulas and therefore, the numerically computed sequence $\mathbb{D}_{J} f$ may no longer fall inside the range of $\mathbb{D}_{J}$. Even if the mapping in (1.6) is onto, it is highly nontrivial to practically implement the discretization in (1.6) using a refinable function $\phi$. On the contrary, a practical sampling device for discretizating a function in the continuum domain is often pre-designed. For example, a digital camera can be used to convert a natural scene from the continuum domain into the digital world. It is more realistic to assume that a digital device employing (1.6) uses a pre-designed function $\phi=\eta$ and a particularly selected integer $J$. Therefore, a discretization or a measurement of $f$ is given in advance by $\left\{\left\langle f, \eta_{\mathrm{M}^{J} ; \mathbf{k}}\right\rangle\right\}_{\mathbf{k} \in \mathbb{Z}^{d}}$, instead of the one in (1.6) using a refinable function $\phi$. These difficulties motivate us to directly study a discrete framelet transform without associating it to an underlying affine system in the function space $L_{2}\left(\mathbb{R}^{d}\right)$. To our best knowledge, almost all papers and books on wavelet analysis deal with wavelets in the function space $L_{2}\left(\mathbb{R}^{d}\right)$ and a discrete framelet transform is always regarded as a consequence of a multiresolution analysis as outlined above. Though it is very natural and fundamental to directly study a discrete framelet/wavelet transform, this algorithmic approach has barely been adopted before in wavelet analysis and harmonic analysis, including books oriented for engineers such as $[39,40]$.

The structure of the paper is as follows. In Section 2, we shall recall the one-level discrete framelet transform and then study its perfect reconstruction property. We shall explain the differences between a discrete framelet transform and its special case-a discrete wavelet transform. In Section 3, we study several basic key properties that are closely related to sparsity of a discrete framelet transform in the discrete setting, in particular, properties such as vanishing moments, sum rules, and polynomial reproduction. In Section 4, we shall introduce the notion of stability of a discrete framelet transform and discrete affine systems. Then we shall study the stability issue of a discrete framelet transform, which is one of the most important and challenging aspects of wavelet analysis. In Section 5 we shall investigate symmetry property of wavelets/framelets and the important role played by linear-phase moments in wavelet analysis. In Section 6, we shall study connections between dual framelet filter banks and frequency-based dual framelets. We show that every dual framelet filter bank naturally corresponds to a frequency-based dual framelet without any a priori condition. Finally, in Section 7 we present an example of directional tight framelets using tensor product of univariate complex-valued tight framelets. 


\section{Perfect Reconstruction of Discrete Framelet Transforms}

In this section, we recall one-level discrete framelet transforms (DFrT) in dimension $d$. Among the three fundamental properties of a discrete framelet transform: prefect reconstruction, sparsity, and stability, we address in this section the most basic property - the perfect reconstruction property.

To introduce a discrete framelet transform, we need some definitions and concepts first. By $l\left(\mathbb{Z}^{d}\right)$ we denote the linear space of all sequences $v: \mathbb{Z}^{d} \rightarrow \mathbb{C}$ of complex numbers on $\mathbb{Z}^{d}$. For $v \in l\left(\mathbb{Z}^{d}\right)$, we often write $v=\{v(\mathrm{k})\}_{\mathrm{k} \in \mathbb{Z}^{d}}$. Theoretically, a discrete input data is often regarded as an element in $l\left(\mathbb{Z}^{d}\right)$. Similarly, by $l_{0}\left(\mathbb{Z}^{d}\right)$ we denote the linear space of all sequences $u=\{u(\mathrm{k})\}_{\mathrm{k} \in \mathbb{Z}^{d}}: \mathbb{Z}^{d} \rightarrow \mathbb{C}$ on $\mathbb{Z}^{d}$ such that $\left\{\mathrm{k} \in \mathbb{Z}^{d}: u(\mathrm{k}) \neq 0\right\}$ is a finite set. An element in $l_{0}\left(\mathbb{Z}^{d}\right)$ is often regarded as a finite-impulse-response (FIR) filter or a finitely supported mask. In this paper, we use $u$ for a general filter and $v$ for a general data.

A discrete framelet transform can be described using two linear operators - the subdivision operator and the transition operator. More precisely, for a filter $u \in l_{0}\left(\mathbb{Z}^{d}\right)$ and a $d \times d$ integer matrix $\mathrm{M}$, the subdivision operator $\mathcal{S}_{u, \mathrm{M}}: l\left(\mathbb{Z}^{d}\right) \rightarrow l\left(\mathbb{Z}^{d}\right)$ and the transition operator $\mathcal{T}_{u, \mathrm{M}}: l\left(\mathbb{Z}^{d}\right) \rightarrow l\left(\mathbb{Z}^{d}\right)$ are defined to be

$$
\begin{array}{ll}
{\left[\mathcal{S}_{u, \mathrm{M}} v\right](\mathrm{n}):=|\operatorname{det}(\mathrm{M})| \sum_{\mathrm{k} \in \mathbb{Z}^{d}} v(\mathrm{k}) u(\mathrm{n}-\mathrm{Mk}),} & \mathrm{n} \in \mathbb{Z}^{d}, \\
{\left[\mathcal{T}_{u, \mathrm{M}} v\right](\mathrm{n}):=|\operatorname{det}(\mathrm{M})| \sum_{\mathrm{k} \in \mathbb{Z}^{d}} v(\mathrm{k}) \overline{u(\mathrm{k}-\mathrm{Mn})},} & \mathrm{n} \in \mathbb{Z}^{d}
\end{array}
$$

for $v \in l\left(\mathbb{Z}^{d}\right)$. The transition operator is used for decomposition and plays the role of coarsening and frequency-separating the data to lower resolution levels; while the subdivision operator is used for reconstruction and plays the role of refining and predicting the data to higher resolution levels.

For a $d \times d$ invertible integer matrix $\mathrm{M}$, we frequently use the following notations:

$$
\mathrm{d}_{\mathrm{M}}:=|\operatorname{det}(\mathrm{M})|, \quad \Gamma_{\mathrm{M}}:=\left(\mathrm{M}[0,1)^{d}\right) \cap \mathbb{Z}^{d} \quad \text { and } \quad \Omega_{\mathrm{M}}:=\left(\left(\mathrm{M}^{\top}\right)^{-1} \mathbb{Z}^{d}\right) \cap[0,1)^{d} .
$$

In other words, $\Gamma_{\mathrm{M}}=\left\{\gamma_{1}, \ldots, \gamma_{\mathrm{d}_{\mathrm{M}}}\right\}$ denotes a complete set of representatives of the distinct cosets of the quotient group $\mathbb{Z}^{d} /\left[\mathrm{M} \mathbb{Z}^{d}\right]$, while $\Omega_{\mathrm{M}}=\left\{\omega_{1}, \ldots, \omega_{\mathrm{d}_{\mathrm{M}}}\right\}$ denotes a complete set of representatives of the distinct cosets of the quotient group $\left[\left(\mathrm{M}^{\top}\right)^{-1} \mathbb{Z}^{d}\right] / \mathbb{Z}^{d}$. Note that $\Omega_{\mathrm{M}}=\left(\mathrm{M}^{\top}\right)^{-1} \Gamma_{\mathrm{M}^{\top}}$.

It is often convenient to use the formal Fourier series (or symbol) $\widehat{v}$ of a sequence $v=\{v(\mathbf{k})\}_{\mathbf{k} \in \mathbb{Z}^{d}}$, which is defined to be $\widehat{v}(\xi):=\sum_{\mathrm{k} \in \mathbb{Z}^{d}} v(\mathrm{k}) e^{-i \mathrm{k} \cdot \xi}$ for $\xi \in \mathbb{R}^{d}$, where $\mathrm{k} \cdot \xi:=k_{1} \xi_{1}+\cdots+k_{d} \xi_{d}$ for $\mathrm{k}=\left(k_{1}, \ldots, k_{d}\right)^{\top}$ and $\xi=\left(\xi_{1}, \ldots, \xi_{d}\right)^{\top}$. Note that $v=\{v(\mathrm{k})\}_{\mathbf{k} \in \mathbb{Z}^{d}}$ is simply the sequence of Fourier coefficients of $\widehat{v}$. Quite often, one only needs to deal with $v$ in the space $l_{2}\left(\mathbb{Z}^{d}\right)$, equipped with the inner product:

$$
\langle v, w\rangle:=\sum_{\mathbf{k} \in \mathbb{Z}^{d}} v(\mathbf{k}) \overline{w(\mathrm{k})}, \quad v, w \in l_{2}\left(\mathbb{Z}^{d}\right)
$$

and $\|v\|_{l_{2}\left(\mathbb{Z}^{d}\right)}^{2}:=\langle v, v\rangle<\infty$. In terms of Fourier series, we have

$$
\widehat{\mathcal{S}_{u, \mathrm{M}} v}(\xi)=\mathrm{d}_{\mathrm{M}} \widehat{v}\left(\mathrm{M}^{\top} \xi\right) \widehat{u}(\xi), \quad \widehat{\mathcal{T}_{u, \mathrm{M}} v}(\xi)=\sum_{\omega \in \Omega_{\mathrm{M}}} \widehat{v}\left(\left(\mathrm{M}^{\top}\right)^{-1} \xi+2 \pi \omega\right) \overline{\widehat{u}\left(\left(\mathrm{M}^{\top}\right)^{-1} \xi+2 \pi \omega\right)} .
$$

We now describe a one-level standard (multidimensional) discrete framelet transform which consists of two parts: a one-level framelet decomposition and a one-level framelet reconstruction. Let $\tilde{u}_{0}, \ldots, \tilde{u}_{s} \in$ $l_{0}\left(\mathbb{Z}^{d}\right)$ be filters for decomposition. For a given data $v \in l\left(\mathbb{Z}^{d}\right)$, a one-level framelet decomposition employing the filter bank $\left\{\tilde{u}_{0}, \ldots, \tilde{u}_{s}\right\}$ is

$$
w_{\ell}:=\mathrm{d}_{\mathrm{M}}^{-1 / 2} \mathcal{T}_{\tilde{u}_{\ell}, \mathrm{M}} v, \quad \ell=0, \ldots, s,
$$


where $w_{\ell}$ are called sequences of framelet coefficients of the input signal $v$. We can group all sequences of framelet coefficients together and define a framelet decomposition (or analysis) operator $\widetilde{\mathcal{W}}: l\left(\mathbb{Z}^{d}\right) \rightarrow$ $\left(l\left(\mathbb{Z}^{d}\right)\right)^{1 \times(s+1)}$ employing the filter bank $\left\{\tilde{u}_{0}, \ldots, \tilde{u}_{s}\right\}$ as follows:

$$
\widetilde{\mathcal{W}} v:=\mathrm{d}_{\mathrm{M}}^{-1 / 2}\left(\mathcal{T}_{\tilde{u}_{0}, \mathrm{M}} v, \ldots, \mathcal{T}_{\tilde{u}_{s}, \mathrm{M}} v\right), \quad v \in l\left(\mathbb{Z}^{d}\right) .
$$

Let $u_{0}, \ldots, u_{s} \in l_{0}\left(\mathbb{Z}^{d}\right)$ be filters for reconstruction. A one-level framelet reconstruction employing the filter bank $\left\{u_{0}, \ldots, u_{s}\right\}$ can be described by a framelet reconstruction (or synthesis) operator $\mathcal{V}:\left(l\left(\mathbb{Z}^{d}\right)\right)^{1 \times(s+1)} \rightarrow l\left(\mathbb{Z}^{d}\right)$ which is defined to be

$$
\mathcal{V}\left(w_{0}, \ldots, w_{s}\right):=\mathrm{d}_{\mathrm{M}}^{-1 / 2} \sum_{\ell=0}^{s} \mathcal{S}_{u_{\ell}, \mathrm{M}} w_{\ell}, \quad w_{0}, \ldots, w_{s} \in l\left(\mathbb{Z}^{d}\right) .
$$

The role played by the factor $\mathrm{d}_{\mathrm{M}}^{-1 / 2}$ in (2.7) and (2.8) is to balance or preserve energy between the input signal and its framelet coefficients. We shall explain this issue later in this section. We denote a framelet decomposition operator employing the filter bank $\left\{u_{0}, \ldots, u_{s}\right\}$ by $\mathcal{W}$ and similarly, a framelet reconstruction operator employing the filter bank $\left\{\tilde{u}_{0}, \ldots, \tilde{u}_{s}\right\}$ by $\widetilde{\mathcal{V}}$.

One of the fundamental properties of a discrete framelet transform is the prefect reconstruction property: $\mathcal{V} \widetilde{\mathcal{W}} v=v$ for all input data $v$. We say that a discrete framelet transform employing a filter bank $\left(\left\{\tilde{u}_{0}, \ldots, \tilde{u}_{s}\right\},\left\{u_{0}, \ldots, u_{s}\right\}\right)$, or simply, a filter bank $\left(\left\{\tilde{u}_{0}, \ldots, \tilde{u}_{s}\right\},\left\{u_{0}, \ldots, u_{s}\right\}\right)$ has the perfect reconstruction (PR) property if $\mathcal{V} \widehat{\mathcal{W}} v=v$ for all input data $v \in l\left(\mathbb{Z}^{d}\right)$. By $\delta$ we denote the Dirac (or Kronecker) sequence such that

$$
\delta(0)=1 \quad \text { and } \quad \delta(\mathrm{k})=0, \quad \forall \mathrm{k} \neq 0 .
$$

The following is a necessary and sufficient condition for the perfect reconstruction property of a general one-level discrete framelet transform:

Theorem 2.1. Let $\tilde{u}_{0}, \ldots, \tilde{u}_{s}, u_{0}, \ldots, u_{s} \in l_{0}\left(\mathbb{Z}^{d}\right)$. Then the following statements are equivalent:

(i) $\left(\left\{\tilde{u}_{0}, \ldots, \tilde{u}_{s}\right\},\left\{u_{0}, \ldots, u_{s}\right\}\right)$ has perfect reconstruction property, that is, for all $v \in l\left(\mathbb{Z}^{d}\right)$,

$$
v=\mathcal{V} \widetilde{\mathcal{W}} v=\mathrm{d}_{\mathrm{M}}^{-1} \sum_{\ell=0}^{s} \mathcal{S}_{u_{\ell}, \mathrm{M}} \mathcal{T}_{\tilde{u}_{\ell}, \mathrm{M}} v
$$

where $\widetilde{\mathcal{W}}$ and $\mathcal{V}$ are defined in $(2.7)$ and $(2.8)$, respectively.

(ii) The identity in (2.10) holds for all $v \in l_{0}\left(\mathbb{Z}^{d}\right)$.

(iii) The identity in (2.10) holds for $\mathrm{d}_{\mathrm{M}}$ particular sequences $v=\delta(\cdot-\gamma), \gamma \in \Gamma_{\mathrm{M}}$.

(iv) The following perfect reconstruction condition holds: for all $\omega \in \Omega_{\mathrm{M}}$ and $\xi \in \mathbb{R}^{d}$,

$$
\widehat{\tilde{u}_{0}}(\xi) \widehat{\widehat{u_{0}}(\xi+2 \pi \omega)}+\widehat{\tilde{u}_{1}}(\xi) \overline{\widehat{u_{1}}(\xi+2 \pi \omega)}+\cdots+\widehat{\tilde{u}_{s}}(\xi) \overline{\widehat{u}_{s}(\xi+2 \pi \omega)}=\delta(\omega) .
$$

Proof. (i) $\Longrightarrow$ (ii) $\Longrightarrow$ (iii) is trivial by $l_{0}\left(\mathbb{Z}^{d}\right) \subseteq l\left(\mathbb{Z}^{d}\right)$. By (2.5), we have

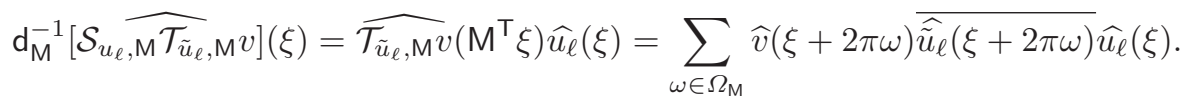

Therefore, (2.10) holds if and only if

$$
\overline{\widehat{v}(\xi)}=\sum_{\omega \in \Omega_{\mathrm{M}}} \overline{\widehat{v}(\xi+2 \pi \omega)} \sum_{\ell=0}^{s} \widehat{\tilde{u}_{\ell}}(\xi+2 \pi \omega) \overline{\widehat{u}_{\ell}(\xi)} .
$$

Plugging $v=\delta(\cdot-\gamma)$ into $(2.13)$ and noting $\widehat{\delta(\cdot-\gamma}(\xi)=e^{-i \gamma \cdot \xi}$, we see that $(2.13)$ becomes

$$
1=\sum_{\omega \in \Omega_{\mathrm{M}}} e^{i \gamma \cdot 2 \pi \omega} \sum_{\ell=0}^{s} \widehat{\tilde{u}}_{\ell}(\xi+2 \pi \omega) \overline{\widehat{u}_{\ell}(\xi)} .
$$


Since $\mathrm{d}_{\mathrm{M}}^{-1 / 2}\left(e^{i \gamma \cdot 2 \pi \omega}\right)_{\gamma \in \Gamma_{\mathrm{M}}, \omega \in \Omega_{\mathrm{M}}}$ is a unitary matrix, we deduce that (2.11) must hold. Therefore, (iii) $\Longrightarrow$ (iv). If (2.11) is satisfied, then (2.13) holds trivially for all $v \in l_{0}\left(\mathbb{Z}^{d}\right)$. Hence, (iv) $\Longrightarrow$ (ii).

To complete the proof, we show that (ii) $\Longrightarrow$ (i). Note that all filters $u_{0}, \ldots, u_{s}, \tilde{u}_{0}, \ldots, \tilde{u}_{s}$ are supported inside a ball $B_{r}(\mathbf{0})$ with center $\mathbf{0}$ and radius $r$. Let $v \in l\left(\mathbb{Z}^{d}\right)$ and $\mathrm{n} \in \mathbb{Z}^{d}$. We shall deduce from (ii) that

$$
\mathrm{d}_{\mathrm{M}}^{-1} \sum_{\ell=0}^{s}\left[\mathcal{S}_{u_{\ell}, \mathrm{M}} \mathcal{T}_{\tilde{u}_{\ell}, \mathrm{M}} v\right](\mathrm{n})=v(\mathrm{n}) .
$$

Define $K_{\mathrm{n}}:=\mathrm{M}^{-1}\left(\mathrm{n}-B_{r}(\mathbf{0})\right)$. Define a finitely supported sequence $v_{\mathrm{n}} \in l_{0}\left(\mathbb{Z}^{d}\right)$ by $v_{\mathrm{n}}(\mathrm{k}):=v(\mathrm{k})$ for all $\mathrm{k} \in \mathbb{Z}^{d} \cap\left(B_{r}(\mathbf{0})+\mathrm{M} K_{\mathrm{n}}\right)$, and $v_{\mathrm{n}}(\mathrm{k})=0$ otherwise. Clearly, $v_{\mathrm{n}} \in l_{0}\left(\mathbb{Z}^{d}\right)$ and $v_{\mathrm{n}}(\mathrm{n})=v(\mathrm{n})$ since $\mathrm{n} \in \mathbb{Z}^{d} \cap\left(B_{r}(\mathbf{0})+\mathrm{M} K_{\mathrm{n}}\right)$. For all $\mathrm{k} \in \mathbb{Z}^{d} \cap K_{\mathrm{n}}$, since all filters are supported inside $B_{r}(\mathbf{0})$,

$$
\left[\mathcal{T}_{\tilde{u}_{\ell}, \mathrm{M}} v\right](\mathrm{k})=\mathrm{d}_{\mathrm{M}} \sum_{\mathrm{j} \in \mathbb{Z}^{d}} v(\mathrm{j}) \overline{\tilde{u}_{\ell}(\mathrm{j}-\mathrm{Mk})}=\mathrm{d}_{\mathrm{M}} \sum_{j \in \mathbb{Z}^{d} \cap\left(B_{r}(\mathbf{0})+\mathrm{M} K_{\mathrm{n}}\right)} v_{\mathrm{n}}(\mathrm{j}) \overline{\tilde{u}_{\ell}(\mathrm{j}-\mathrm{Mk})}=\left[\mathcal{T}_{\tilde{u}_{\ell}, \mathrm{M}} v_{\mathrm{n}}\right](\mathrm{k}) .
$$

Therefore, we deduce that

$$
\begin{aligned}
& \mathrm{d}_{\mathrm{M}}^{-1} \sum_{\ell=0}^{s}\left[\mathcal{S}_{u_{\ell}, \mathrm{M}} \mathcal{T}_{\tilde{u}_{\ell}, \mathrm{M}} v\right](\mathrm{n})=\sum_{\ell=0}^{s} \sum_{\mathrm{k} \in \mathbb{Z}^{d} \cap K_{\mathrm{n}}}\left[\mathcal{T}_{\tilde{u}_{\ell}, \mathrm{M}} v\right](\mathrm{k}) u_{\ell}(\mathrm{n}-\mathrm{Mk}) \\
& =\sum_{\ell=0}^{s} \sum_{\mathrm{k} \in \mathbb{Z}^{d} \cap K_{\mathrm{n}}}\left[\mathcal{T}_{\tilde{u}_{\ell}, \mathrm{M}} v_{\mathrm{n}}\right](\mathrm{k}) u_{\ell}(\mathrm{n}-\mathrm{Mk})=\mathrm{d}_{\mathrm{M}}^{-1} \sum_{\ell=0}^{s}\left[\mathcal{S}_{u_{\ell}, \mathrm{M}} \mathcal{T}_{\tilde{u}_{\ell}, \mathrm{M}} v_{\mathrm{n}}\right](\mathrm{n})=v_{\mathrm{n}}(\mathrm{n})=v(\mathrm{n}),
\end{aligned}
$$

where we used (ii) and the fact that $v_{\mathrm{n}}(\mathrm{n})=v(\mathrm{n})$. Hence, (ii) $\Longrightarrow(\mathrm{i})$.

The condition in (2.11) can be equivalently rewritten as the following matrix form:

$$
\left[\begin{array}{ccc}
\widehat{u_{0}}\left(\xi+2 \pi \omega_{1}\right) & \cdots & \widehat{u_{s}}\left(\xi+2 \pi \omega_{1}\right) \\
\vdots & \ddots & \vdots \\
\widehat{u_{0}}\left(\xi+2 \pi \omega_{\mathrm{d}_{\mathrm{M}}}\right) & \cdots & {\widehat{u_{s}}}_{s}\left(\xi+2 \pi \omega_{\mathrm{d}_{\mathrm{M}}}\right)
\end{array}\right]\left[\begin{array}{ccc}
\widehat{u_{0}}\left(\xi+2 \pi \omega_{1}\right) & \cdots & \widehat{u_{s}}\left(\xi+2 \pi \omega_{1}\right) \\
\vdots & \ddots & \vdots \\
\widehat{u_{0}}\left(\xi+2 \pi \omega_{\mathrm{d}_{\mathrm{M}}}\right) & \cdots & \widehat{u_{s}}\left(\xi+2 \pi \omega_{\mathrm{d}_{\mathrm{M}}}\right)
\end{array}\right]^{\star}=I_{\mathrm{d}_{\mathrm{M}}},
$$

where $\left\{\omega_{1}, \ldots, \omega_{\mathrm{d}_{\mathrm{M}}}\right\}:=\Omega_{\mathrm{M}}$. A filter bank satisfying the perfect reconstruction condition in (2.15) is called a dual $\mathrm{M}$-framelet filter bank. It follows trivially from the perfect reconstruction condition in (2.15) that $\left(\left\{\tilde{u}_{0}, \ldots, \tilde{u}_{s}\right\},\left\{u_{0}, \ldots, u_{s}\right\}\right)$ is a dual $\mathrm{M}$-framelet filter bank if and only if $\left(\left\{u_{0}, \ldots, u_{s}\right\},\left\{\tilde{u}_{0}, \ldots, \tilde{u}_{s}\right\}\right)$ is a dual $\mathrm{M}$-framelet filter bank. In other words, $\mathcal{V} \widetilde{\mathcal{W}}=\operatorname{Id}_{l(\mathbb{Z})}$ if and only if $\widetilde{\mathcal{V}} \mathcal{W}=\operatorname{Id}_{l(\mathbb{Z})}$. In particular, a dual M-framelet filter bank with $s=\mathrm{d}_{\mathrm{M}}-1$ is called a biorthogonal $\mathrm{M}$-wavelet filter bank which, by the following result, is a nonredundant filter bank.

Proposition 2.2. Let $\left(\left\{\tilde{u}_{0}, \ldots, \tilde{u}_{s}\right\},\left\{u_{0}, \ldots, u_{s}\right\}\right)$ be a dual $\mathrm{M}$-framelet filter bank. Let the framelet decomposition operator $\widetilde{\mathcal{W}}: l\left(\mathbb{Z}^{d}\right) \rightarrow\left(l\left(\mathbb{Z}^{d}\right)\right)^{1 \times(s+1)}$ and framelet reconstruction operator $\mathcal{V}:$ $\left(l\left(\mathbb{Z}^{d}\right)\right)^{1 \times(s+1)} \rightarrow l\left(\mathbb{Z}^{d}\right)$ be defined in $(2.7)$ and (2.8). Then the following statements are equivalent:

(i) $\widetilde{\mathcal{W}}$ is onto, or $\mathcal{V}$ is one-to-one.

(ii) $\mathcal{V W}=\operatorname{Id}_{l\left(\mathbb{Z}^{d}\right)}$ and $\widetilde{\mathcal{W}} \mathcal{V}=\operatorname{Id}_{\left(l\left(\mathbb{Z}^{d}\right)\right)^{1 \times(s+1)}}$, that is, $\mathcal{V}^{-1}=\widetilde{\mathcal{W}}$ and $\widetilde{\mathcal{W}}^{-1}=\mathcal{V}$.

(iii) $s=\mathrm{d}_{\mathrm{M}}-1$, where $\mathrm{d}_{\mathrm{M}}:=|\operatorname{det}(\mathrm{M})|$.

The same conclusion holds if $l\left(\mathbb{Z}^{d}\right)$ is replaced by $l_{2}\left(\mathbb{Z}^{d}\right)$ or $l_{0}\left(\mathbb{Z}^{d}\right)$.

Proof. (ii) $\Longrightarrow(\mathrm{i})$ is trivial. Note that $\mathcal{V} \widetilde{\mathcal{W}}=\operatorname{Id}_{l\left(\mathbb{Z}^{d}\right)}$ follows directly from the perfect reconstruction property. Using $\widetilde{\mathcal{W}} \mathcal{V} \widetilde{\mathcal{W}} w=\widetilde{\mathcal{W}} w$, if $\widetilde{\mathcal{W}}$ is onto, then $\widetilde{\mathcal{W}}\left(l\left(\mathbb{Z}^{d}\right)\right)^{1 \times(s+1)}=l\left(\mathbb{Z}^{d}\right)$ and we must have $\widetilde{\mathcal{W}} \mathcal{V}=$ $\operatorname{Id}_{\left(l\left(\mathbb{Z}^{d}\right)\right)^{1 \times(s+1)}}$. By $\mathcal{V} \widetilde{\mathcal{W}}=\operatorname{Id}_{l\left(\mathbb{Z}^{d}\right)}$, we have $\mathcal{V} \widetilde{\mathcal{V}} \mathcal{V}=\mathcal{V}$ and therefore, $\mathcal{V}\left(\widetilde{\mathcal{W}} \mathcal{V}-\operatorname{Id}_{\left(l\left(\mathbb{Z}^{d}\right)\right)^{1 \times(s+1)}}\right)=0$. If $\mathcal{V}$ is one-to-one, we must have $\widetilde{\mathcal{W}} \mathcal{V}=\operatorname{Id}_{\left(l\left(\mathbb{Z}^{d}\right)\right)^{1 \times(s+1)}}$. Hence, (i) $\Longrightarrow$ (ii). 
Now we prove (ii) $\Longleftrightarrow$ (iii). Suppose that (ii) holds. Then $\widetilde{\mathcal{W}} \mathcal{V}=\operatorname{Id}_{\left(l\left(\mathbb{Z}^{d}\right)\right)^{1 \times(s+1)}}$ implies

$$
\mathrm{d}_{\mathrm{M}}^{-1} \mathcal{T}_{\tilde{u}_{\ell}, \mathrm{M}}\left(\mathcal{S}_{u_{0}, \mathrm{M}} w_{0}+\cdots+\mathcal{S}_{u_{s}, \mathrm{M}} w_{s}\right)=w_{\ell}, \quad \forall w_{0}, \ldots, w_{s} \in l\left(\mathbb{Z}^{d}\right), \ell=0, \ldots, s .
$$

Taking the Fourier series on both sides of (2.16), we see that (2.16) is equivalent to

$$
\begin{aligned}
& \widehat{w_{0}}(\xi) \sum_{\omega \in \Omega_{\mathrm{M}}} \widehat{u_{0}}\left(\left(\mathrm{M}^{\top}\right)^{-1} \xi+2 \pi \omega\right) \overline{\hat{\tilde{u}}_{\ell}\left(\left(\mathrm{M}^{\top}\right)^{-1} \xi+2 \pi \omega\right)}+\cdots \\
& \quad+\widehat{w_{s}}(\xi) \sum_{\omega \in \Omega_{\mathrm{M}}} \widehat{u_{s}}\left(\left(\mathrm{M}^{\top}\right)^{-1} \xi+2 \pi \omega\right) \overline{\hat{\tilde{u}}_{\ell}\left(\left(\mathrm{M}^{\top}\right)^{-1} \xi+2 \pi \omega\right)}=\widehat{w_{\ell}}(\xi)
\end{aligned}
$$

for all $\ell=0, \ldots, s$. It is trivial to see that the above identities hold if and only if

$$
\sum_{\omega \in \Omega_{\mathrm{M}}} \widehat{\widehat{u_{m}}\left(\left(\mathrm{M}^{\top}\right)^{-1} \xi+2 \pi \omega\right)} \widehat{\tilde{u}_{\ell}}\left(\left(\mathrm{M}^{\top}\right)^{-1} \xi+2 \pi \omega\right)=\delta(\ell-m), \quad \ell, m=0, \ldots, s .
$$

Rewriting the above identities into matrix form, we see that $\widetilde{\mathcal{W}} \mathcal{V}=\operatorname{Id}_{\left(l\left(\mathbb{Z}^{d}\right)\right)^{1 \times(s+1)}}$ if and only if

$$
\left[\begin{array}{ccc}
\widehat{u_{0}}\left(\xi+2 \pi \omega_{1}\right) & \cdots & \widehat{u_{s}}\left(\xi+2 \pi \omega_{1}\right) \\
\vdots & \ddots & \vdots \\
\widehat{u_{0}}\left(\xi+2 \pi \omega_{\mathrm{d}_{\mathrm{M}}}\right) & \cdots & \widehat{u_{s}}\left(\xi+2 \pi \omega_{\mathrm{d}_{\mathrm{M}}}\right)
\end{array}\right]^{\star}\left[\begin{array}{ccc}
\widehat{\tilde{u}_{0}}\left(\xi+2 \pi \omega_{1}\right) & \cdots & \widehat{\tilde{u}_{s}}\left(\xi+2 \pi \omega_{1}\right) \\
\vdots & \ddots & \vdots \\
\widehat{\tilde{u}_{0}}\left(\xi+2 \pi \omega_{\mathrm{d}_{\mathrm{M}}}\right) & \cdots & \widehat{\tilde{u}_{s}}\left(\xi+2 \pi \omega_{\mathrm{d}_{\mathrm{M}}}\right)
\end{array}\right]=I_{s+1} .
$$

Combining (2.17) with (2.15), we conclude that we must have $s+1=\mathrm{d}_{\mathrm{M}}$. Therefore, (ii) $\Longrightarrow$ (iii).

Conversely, if $s=\mathrm{d}_{\mathrm{M}}-1$, as a square matrix, (2.15) directly implies (2.17). By the above argument, we must have $\widetilde{\mathcal{W}} \mathcal{V}=\operatorname{Id}_{\left(l\left(\mathbb{Z}^{d}\right)\right)^{1 \times(s+1)}}$. Therefore, (iii) must hold.

If $l\left(\mathbb{Z}^{d}\right)$ is replaced by $S:=l_{2}\left(\mathbb{Z}^{d}\right)$ or $l_{0}\left(\mathbb{Z}^{d}\right)$, one can check that $\mathcal{W}: S \rightarrow(S)^{1 \times(s+1)}$ and $\tilde{\mathcal{V}}$ : $(S)^{1 \times(s+1)} \rightarrow S$ are well defined. The same proof can be used to verify the claims.

Consequently, under a biorthogonal wavelet filter bank, any input signal $v \in l_{2}\left(\mathbb{Z}^{d}\right)$ has a nonredundant representation $v=\mathcal{V} w$ with the unique choice $w=\widetilde{\mathcal{W}} v$; while under a dual framelet filter bank with $s \geqslant \mathrm{~d}_{\mathrm{M}}$, an input signal $v$ can be represented as $v=\mathcal{V} w$ from infinitely many $w \in\left(l\left(\mathbb{Z}^{d}\right)\right)^{1 \times(s+1)}$ of framelet coefficients.

In the following, we explain the role played by the factor $\mathrm{d}_{\mathrm{M}}^{-1 / 2}$ in $(2.7)$ and (2.8). To do so, we need the following simple relation, which is well known in the literature (e.g., see [5]), between the subdivision operator $\mathcal{S}_{u, \mathrm{M}}$ and the transition operator $\mathcal{T}_{u, \mathrm{M}}$ acting on the space $l_{2}\left(\mathbb{Z}^{d}\right)$.

Lemma 2.3. Let $u \in l_{0}\left(\mathbb{Z}^{d}\right)$ be a finitely supported filter on $\mathbb{Z}^{d}$. Then $\mathcal{S}_{u, \mathrm{M}}: l_{2}\left(\mathbb{Z}^{d}\right) \rightarrow l_{2}\left(\mathbb{Z}^{d}\right)$ is the adjoint operator of $\mathcal{T}_{u, \mathrm{M}}: l_{2}\left(\mathbb{Z}^{d}\right) \rightarrow l_{2}\left(\mathbb{Z}^{d}\right)$; that is, $\mathcal{T}_{u, \mathrm{M}}^{\star}=\mathcal{S}_{u, \mathrm{M}}$ satisfying

$$
\left\langle\mathcal{S}_{u, \mathrm{M}} v, w\right\rangle=\left\langle\mathcal{T}_{u, \mathrm{M}}^{\star} v, w\right\rangle:=\left\langle v, \mathcal{T}_{u, \mathrm{M}} w\right\rangle, \quad \forall v, w \in l_{2}\left(\mathbb{Z}^{d}\right) .
$$

Proof. By (2.5), we have $\widehat{\mathcal{S}_{u, \mathrm{M}} v}(\xi)=\mathrm{d}_{\mathrm{M}} \widehat{v}\left(\mathrm{M}^{\top} \xi\right) \widehat{u}(\xi)$ and hence

$$
\begin{aligned}
\left\langle\mathcal{S}_{u, \mathrm{M}} v, w\right\rangle & =\frac{\mathrm{d}_{\mathrm{M}}}{(2 \pi)^{d}} \int_{[-\pi, \pi)^{d}} \widehat{v}\left(\mathrm{M}^{\top} \xi\right) \widehat{u}(\xi) \widehat{\widehat{w}(\xi)} d \xi \\
& =\frac{1}{(2 \pi)^{d}} \int_{\mathrm{M}^{\top}[-\pi, \pi)^{d}} \widehat{v}(\xi) \widehat{u}\left(\left(\mathrm{M}^{\top}\right)^{-1} \xi\right) \overline{\widehat{w}\left(\left(\mathrm{M}^{\top}\right)^{-1} \xi\right)} d \xi=\frac{1}{(2 \pi)^{d}} \int_{[-\pi, \pi)^{d}} \widehat{v}(\xi) \overline{\mathcal{T}_{u}, \mathrm{M} w(\xi)} d \xi
\end{aligned}
$$

where we used (2.5) in the last identity. Hence, (2.18) holds. 
Note that the space $\left(l_{2}\left(\mathbb{Z}^{d}\right)\right)^{1 \times(s+1)}$ is equipped with the following inner product:

$$
\left\langle\left(w_{0}, \ldots, w_{s}\right),\left(\tilde{w}_{0}, \ldots, \tilde{w}_{s}\right)\right\rangle:=\left\langle w_{0}, \tilde{w}_{0}\right\rangle+\cdots+\left\langle w_{s}, \tilde{w}_{s}\right\rangle, \quad w_{0}, \ldots, w_{s}, \tilde{w}_{0}, \ldots, \tilde{w}_{s} \in l_{2}\left(\mathbb{Z}^{d}\right)
$$

and $\left\|\left(w_{0}, \ldots, w_{s}\right)\right\|_{\left(l_{2}\left(\mathbb{Z}^{d}\right)\right)^{1 \times(s+1)}}^{2}:=\left\|w_{0}\right\|_{l_{2}\left(\mathbb{Z}^{d}\right)}^{2}+\cdots+\left\|w_{s}\right\|_{l_{2}\left(\mathbb{Z}^{d}\right)}^{2}$. Recall that

$$
\mathcal{W}: l_{2}\left(\mathbb{Z}^{d}\right) \rightarrow\left(l_{2}\left(\mathbb{Z}^{d}\right)\right)^{1 \times(s+1)} \quad \text { with } \quad \mathcal{W} v:=\mathrm{d}_{\mathrm{M}}^{-1 / 2}\left(\mathcal{T}_{u_{0}, \mathrm{M}} v, \ldots, \mathcal{T}_{u_{s}, \mathrm{M}} v\right), \quad v \in l_{2}\left(\mathbb{Z}^{d}\right)
$$

and

$$
\mathcal{V}:\left(l_{2}\left(\mathbb{Z}^{d}\right)\right)^{1 \times(s+1)} \rightarrow l_{2}\left(\mathbb{Z}^{d}\right) \quad \text { with } \quad \mathcal{V}\left(w_{0}, \ldots, w_{s}\right):=\mathrm{d}_{\mathrm{M}}^{-1 / 2} \sum_{\ell=0}^{s} \mathcal{S}_{u_{\ell}, \mathrm{M}} w_{\ell},
$$

for $w_{0}, \ldots, w_{s} \in l_{2}\left(\mathbb{Z}^{d}\right)$. The adjoint operators of $\mathcal{W}$ and $\mathcal{V}$ are defined to be

$$
\mathcal{W}^{\star}:\left(l_{2}\left(\mathbb{Z}^{d}\right)\right)^{1 \times(s+1)} \rightarrow l_{2}\left(\mathbb{Z}^{d}\right) \quad \text { through } \quad\left\langle v, \mathcal{W}^{\star} w\right\rangle:=\langle\mathcal{W} v, w\rangle
$$

and

$$
\mathcal{V}^{\star}: l_{2}\left(\mathbb{Z}^{d}\right) \rightarrow\left(l_{2}\left(\mathbb{Z}^{d}\right)\right)^{1 \times(s+1)} \quad \text { through } \quad\left\langle\mathcal{V}^{\star} v, w\right\rangle:=\langle v, \mathcal{V} w\rangle
$$

for all $v \in l_{2}\left(\mathbb{Z}^{d}\right)$ and $w \in\left(l_{2}\left(\mathbb{Z}^{d}\right)\right)^{1 \times(s+1)}$. By Lemma 2.3 , we have $\mathcal{W}^{\star}=\mathcal{V}$ and $\mathcal{V}^{\star}=\mathcal{W}$.

The role played by the factor $\mathrm{d}_{\mathrm{M}}^{-1 / 2}$ in $(2.7)$ and (2.8) is explained by the following result:

Theorem 2.4. Let $u_{0}, \ldots, u_{s} \in l_{0}\left(\mathbb{Z}^{d}\right)$ be finitely supported sequences on $\mathbb{Z}^{d}$. Let $\mathcal{W}: l_{2}\left(\mathbb{Z}^{d}\right) \rightarrow$ $\left(l_{2}\left(\mathbb{Z}^{d}\right)\right)^{1 \times(s+1)}$ be defined in (2.19). Then the following statements are equivalent:

(i) $\|\mathcal{W} v\|_{\left(l_{2}\left(\mathbb{Z}^{d}\right)\right)^{1 \times(s+1)}}^{2}=\|v\|_{l_{2}\left(\mathbb{Z}^{d}\right)}^{2}$ for all $v \in l_{2}\left(\mathbb{Z}^{d}\right)$, that is,

$$
\left\|\mathcal{T}_{u_{0}, \mathrm{M}} v\right\|_{l_{2}\left(\mathbb{Z}^{d}\right)}^{2}+\cdots+\left\|\mathcal{T}_{u_{s}, \mathrm{M}} v\right\|_{l_{2}\left(\mathbb{Z}^{d}\right)}^{2}=\mathrm{d}_{\mathrm{M}}\|v\|_{l_{2}\left(\mathbb{Z}^{d}\right)}^{2}, \quad \forall v \in l_{2}\left(\mathbb{Z}^{d}\right) .
$$

(ii) $\langle\mathcal{W} v, \mathcal{W} \tilde{v}\rangle=\langle v, \tilde{v}\rangle$ for all $v, \tilde{v} \in l_{2}\left(\mathbb{Z}^{d}\right)$.

(iii) $\mathcal{W}^{\star} \mathcal{W}=\operatorname{Id}_{l_{2}\left(\mathbb{Z}^{d}\right)}$, that is, $\mathcal{W}^{\star} \mathcal{W} v=v$ for all $v \in l_{2}\left(\mathbb{Z}^{d}\right)$.

(iv) $\left(\left\{u_{0}, \ldots, u_{s}\right\},\left\{u_{0}, \ldots, u_{s}\right\}\right)$ is a dual $\mathrm{M}$-framelet filter bank.

Proof. Obviously, (ii) $\Longrightarrow\left(\right.$ i). Note that (i) implies $\left\langle\mathcal{W}^{\star} \mathcal{W} v, v\right\rangle=\langle\mathcal{W} v, \mathcal{W} v\rangle=\langle v, v\rangle$. Using the wellknown polarization identity, it is straightforward to see that $\langle\mathcal{W} v, \mathcal{W} \tilde{v}\rangle=\left\langle\mathcal{W}^{\star} \mathcal{W} v, \tilde{v}\right\rangle=\langle v, \tilde{v}\rangle$. Hence, (i) $\Longrightarrow$ (ii). The equivalence between (ii) and (iii) is trivial. Note that $\mathcal{W}^{\star}=\mathcal{V}$. The equivalence between (iii) and (iv) follows directly from Theorem 2.1.

A filter bank $\left\{u_{0}, \ldots, u_{s}\right\}$ is called a tight $\mathrm{M}$-framelet filter bank if $\left(\left\{u_{0}, \ldots, u_{s}\right\},\left\{u_{0}, \ldots, u_{s}\right\}\right)$ is a dual $\mathrm{M}$-framelet filter bank. In particular, a tight $\mathrm{M}$-framelet filter bank with $s=\mathrm{d}_{\mathrm{M}}-1$ is called an orthogonal M-wavelet filter bank. By item (i) of Theorem 2.4, the energy is preserved after a tight framelet decomposition: $\sum_{\ell=0}^{s}\left\|w_{\ell}\right\|_{l_{2}\left(\mathbb{Z}^{d}\right)}^{2}=\|\mathcal{W} v\|_{l_{2}\left(\mathbb{Z}^{d}\right)}^{2}=\|v\|_{l_{2}\left(\mathbb{Z}^{d}\right)}^{2}$ for all $v \in l_{2}\left(\mathbb{Z}^{d}\right)$, where $\left(w_{0}, \ldots, w_{s}\right):=\mathcal{W} v$ is the sequence of framelet coefficients. Let $\left\{u_{0}, \ldots, u_{s}\right\}$ be an orthogonal M-wavelet filter bank. Define $\mathcal{W}$ as in (2.19) and $\mathcal{V}$ as in (2.20). By Proposition 2.2 and Theorem 2.4, we see that $\mathcal{W}=\mathcal{V}^{\star}$ is an invertible orthogonal mapping satisfying $\langle\mathcal{W} v, \mathcal{W} \tilde{v}\rangle=\langle v, \tilde{v}\rangle$ for all $v, \tilde{v} \in l_{2}\left(\mathbb{Z}^{d}\right)$ and $\mathcal{V}=\mathcal{W}^{\star}$ is an invertible orthogonal mapping such that for all $w_{0}, \ldots, w_{s}, \tilde{w}_{0}, \ldots, \tilde{w}_{s} \in l_{2}\left(\mathbb{Z}^{d}\right),\left\langle\mathcal{V}\left(w_{0}, \ldots, w_{s}\right), \mathcal{V}\left(\tilde{w}_{0}, \ldots, \tilde{w}_{s}\right)\right\rangle=$ $\left\langle\left(w_{0}, \ldots, w_{s}\right),\left(\tilde{w}_{0}, \ldots, \tilde{w}_{s}\right)\right\rangle$.

\section{Sparsity of Discrete Framelet Transforms}

One key feature of wavelets in the function space $L_{2}\left(\mathbb{R}^{d}\right)$ is the sparse representation for smooth or piecewise smooth functions $([2,8,35,36])$. Since we deal with the discrete setting, the sparsity of a discrete framelet transform, which we shall discuss in this section, refers to the sparsity of framelet coefficients for smooth discrete signals. We shall mention in Section 6 some connections between sparsity of a discrete 
framelet transform and the sparse representation of a framelet/wavelet in the function space $L_{2}\left(\mathbb{R}^{d}\right)$. In applications, it is desirable to have as many as possible negligible framelet coefficients for smooth signals. In this section, we study several basic key mathematical properties that are closely related to sparsity of a discrete framelet transform in the discrete setting, in particular, properties such as vanishing moments, sum rules, and polynomial reproduction. This section is mainly surveyed from the papers $[24,25]$ as well as $[16,19]$. For the convenience of the reader, we shall provide self-contained proofs for results stated in this section.

For an integer $j=1, \ldots, d$, by $\partial_{j}$ we denote the partial derivative with respect to the $j$ th coordinate of $\mathbb{R}^{d}$. Define $\mathbb{N}_{0}:=\mathbb{N} \cup\{0\}$. For $\mu=\left(\mu_{1}, \ldots, \mu_{d}\right)^{\top} \in \mathbb{N}_{0}^{d}$ and $x=\left(x_{1}, \ldots, x_{d}\right)^{\top} \in \mathbb{R}^{d}$, we define $|\mu|:=\left|\mu_{1}\right|+\cdots+\left|\mu_{d}\right|, \mu !:=\mu_{1} ! \cdots \mu_{d} !, x^{\mu}:=x_{1}^{\mu_{1}} \cdots x_{d}^{\mu_{d}}$, and $\partial^{\mu}:=\partial_{1}^{\mu_{1}} \cdots \partial_{d}^{\mu_{d}}$. Moreover, we write $\partial:=\left(\partial_{1}, \ldots, \partial_{d}\right)^{\top}$, the gradient differentiation operator.

Smooth signals are modeled by polynomials of various degrees. Let $\mathrm{p}: \mathbb{R}^{d} \rightarrow \mathbb{C}$ be a $d$-variate polynomial, that is, $\mathrm{p}(x)=\sum_{\mu \in \mathbb{N}_{0}^{d},|\mu| \leqslant n} p_{\mu} x^{\mu}$ for some $n \in \mathbb{N}_{0}$ and if $\sum_{|\mu|=n}\left|p_{\mu}\right| \neq 0, \operatorname{deg}(\mathrm{p}):=n$ is called the (total) degree of the polynomial $p$. Sampling a polynomial $\mathrm{p}$ on the integer lattice $\mathbb{Z}^{d}$, we have a polynomial sequence $\left.\mathrm{p}\right|_{\mathbb{Z}^{d}}: \mathbb{Z}^{d} \rightarrow \mathbb{C}$ which is given by $\left[\left.\mathrm{p}\right|_{\mathbb{Z}^{d}}\right](\mathrm{k})=\mathrm{p}(\mathrm{k}), \mathrm{k} \in \mathbb{Z}^{d}$. If a sequence $v=\{v(\mathrm{k})\}_{\mathrm{k} \in \mathbb{Z}^{d}}$ is a polynomial sequence, then a polynomial $\mathrm{p}$, satisfying $v(\mathrm{k})=\mathrm{p}(\mathrm{k})$ for all $\mathrm{k} \in \mathbb{Z}^{d}$, is uniquely determined. For simplicity of presentation, we shall use $\mathrm{p}$ to denote both a polynomial function $\mathrm{p}$ on $\mathbb{R}^{d}$ and its induced polynomial sequence $\left.\mathrm{p}\right|_{\mathbb{Z}^{d}}$ on $\mathbb{Z}^{d}$.

For a nonnegative integer $m \in \mathbb{N}_{0}, \Pi_{m}$ denotes the space of all polynomials of (total) degree no more than $m$. In particular, $\Pi:=\cup_{m=0}^{\infty} \Pi_{m}$ denotes the space of all polynomials on $\mathbb{R}^{d}$. For a polynomial $\mathrm{p}(x)=\sum_{\mu \in \mathbb{N}_{0}^{d}} p_{\mu} x^{\mu}$ and a smooth function $\mathbf{f}(\xi)$, we use the following polynomial differentiation operator in this section:

$$
\mathrm{p}(x-i \partial) \mathbf{f}(\xi):=\sum_{\mu \in \mathbb{N}_{0}^{d}} p_{\mu}(x-i \partial)^{\mu} \mathbf{f}(\xi),
$$

where in case of confusion $\partial$ is always with respect to $\xi$ in this paper. Using the Taylor expansion of $\mathrm{p}(y+z)$ at the point $y$, we have $\mathrm{p}(y+z)=\sum_{\mu \in \mathbb{N}_{0}^{d}}\left(\partial^{\mu} \mathrm{p}\right)(y) \frac{z^{\mu}}{\mu !}$. Consequently, we have

$$
\mathrm{p}(x-i \partial) \mathbf{f}(\xi)=\sum_{\mu \in \mathbb{N}_{0}^{d}} \frac{(-i)^{|\mu|}}{\mu !} \partial^{\mu} \mathrm{p}(x) \partial^{\mu} \mathbf{f}(\xi)=\sum_{\mu \in \mathbb{N}_{0}^{d}} \frac{x^{\mu}}{\mu !}\left[\left(\partial^{\mu} \mathrm{p}\right)(-i \partial)\right] \mathbf{f}(\xi) .
$$

Using Leibniz differentiation formula and (3.2), one can also verify the following generalized product rule for differentiation:

$$
\mathrm{p}(x-i \partial)(\mathbf{g}(\xi) \mathbf{f}(\xi))=\sum_{\mu \in \mathbb{N}_{0}^{d}} \frac{(-i)^{|\mu|}}{\mu !} \partial^{\mu} \mathbf{g}(\xi)\left[\left(\partial^{\mu} \mathrm{p}\right)(x-i \partial)\right] \mathbf{f}(\xi) .
$$

It follows directly from (3.2) and (3.3) that

$$
\left.\left[\mathrm{p}(-i \partial)\left(e^{i x \cdot \xi} \mathbf{f}(\xi)\right)\right]\right|_{\xi=0}=\left.[\mathrm{p}(x-i \partial) \mathbf{f}(\xi)]\right|_{\xi=0} .
$$

For $u \in l_{0}\left(\mathbb{Z}^{d}\right)$ and $v \in l\left(\mathbb{Z}^{d}\right)$, the convolution $u * v$ satisfying the relation $\widehat{u * v}(\xi)=\widehat{u}(\xi) \widehat{v}(\xi)$ is defined to be

$$
[u * v](\mathrm{n}):=\sum_{\mathbf{k} \in \mathbb{Z}^{d}} u(\mathrm{k}) v(\mathrm{n}-\mathbf{k}), \quad \mathrm{n} \in \mathbb{Z}^{d}
$$

and an associated sequence $v^{\star}$ satisfying the relation $\widehat{v^{\star}}(\xi)=\overline{\widehat{v}(\xi)}$ by

$$
v^{\star}(\mathrm{k}):=\overline{v(-\mathrm{k})}, \quad \mathrm{k} \in \mathbb{Z}^{d} .
$$

To study basic properties of a discrete framelet transform, it is of importance to investigate how the subdivision operator and the transition operator act on polynomial spaces. Since the subdivision and transition operators can be expressed via the convolution operation, in the following we first study the convolution operation acting on polynomial spaces. 
Lemma 3.1. ([25, Proposition 2.1] and [24, (4.7)]) Let $u=\{u(\mathrm{k})\}_{\mathbf{k} \in \mathbb{Z}^{d}} \in l_{0}\left(\mathbb{Z}^{d}\right)$. Then for any d-variate polynomial $\mathrm{p} \in \Pi, \mathrm{p} * u$ is a polynomial sequence with $\operatorname{deg}(\mathrm{p} * u) \leqslant \operatorname{deg}(\mathrm{p})$ and

$$
[\mathrm{p} * u](x):=\sum_{\mathbf{k} \in \mathbb{Z}^{d}} \mathrm{p}(x-\mathrm{k}) u(\mathrm{k})=\sum_{\mu \in \mathbb{N}_{0}^{d}} \frac{(-i)^{|\mu|}}{\mu !} \partial^{\mu} \mathrm{p}(x) \partial^{\mu} \widehat{u}(0)=\left.[\mathrm{p}(x-i \partial) \widehat{u}(\xi)]\right|_{\xi=0} .
$$

Moreover, $\left[\partial^{\mu} \mathrm{p}\right] * u=\partial^{\mu}[\mathrm{p} * u]$ for all $\mu \in \mathbb{N}_{0}^{d}$ and $\mathrm{p}(\cdot-y) * u=[\mathrm{p} * u](\cdot-y)$ for all $y \in \mathbb{R}^{d}$.

Proof. Using the Taylor expansion, we have $\mathrm{p}(x-\mathrm{k})=\sum_{\mu \in \mathbb{N}_{0}^{d}} \partial^{\mu} \mathrm{p}(x) \frac{(-\mathrm{k})^{\mu}}{\mu !}$. Hence, we deduce

$$
[\mathrm{p} * u](x)=\sum_{\mathrm{k} \in \mathbb{Z}^{d}} \mathrm{p}(x-\mathrm{k}) u(\mathrm{k})=\sum_{\mathrm{k} \in \mathbb{Z}^{d}} \sum_{\mu \in \mathbb{N}_{0}^{d}} \partial^{\mu} \mathrm{p}(x) u(\mathrm{k}) \frac{(-\mathrm{k})^{\mu}}{\mu !}=\sum_{\mu \in \mathbb{N}_{0}^{d}} \partial^{\mu} \mathrm{p}(x) \sum_{\mathrm{k} \in \mathbb{Z}^{d}} u(\mathrm{k}) \frac{(-\mathrm{k})^{\mu}}{\mu !} .
$$

By $\widehat{u}(\xi)=\sum_{\mathbf{k} \in \mathbb{Z}^{d}} u(\mathbf{k}) e^{-i \mathbf{k} \cdot \xi}$, we observe that $\partial^{\mu} \widehat{u}(0)=i^{|\mu|} \sum_{\mathbf{k} \in \mathbb{Z}^{d}} u(\mathbf{k})(-\mathbf{k})^{\mu}$. Hence, $[\mathrm{p} * u](x)=$ $\sum_{\mu \in \mathbb{N}_{0}^{d}} \frac{(-i)^{|\mu|}}{\mu !} \partial^{\mu} \mathrm{p}(x) \partial^{\mu} \widehat{u}(0)$, from which and (3.2) we see that all the claims hold.

For an integer $m \in \mathbb{N}_{0}$ and smooth functions $\mathbf{f}$, g, we use the following big $\mathcal{O}$ notation

$$
\mathbf{f}(\xi)=\mathbf{g}(\xi)+\mathcal{O}\left(\left\|\xi-\xi_{0}\right\|^{m}\right), \quad \xi \rightarrow \xi_{0}
$$

to mean the following relation:

$$
\partial^{\mu} \mathbf{f}\left(\xi_{0}\right)=\partial^{\mu} \mathbf{g}\left(\xi_{0}\right), \quad \forall \mu \in \mathbb{N}_{0}^{d} \text { satisfying }|\mu|<m .
$$

For a polynomial $\mathrm{p} \in \Pi_{m-1}$ of degree less than $m$, by (3.7), it is evident that the polynomial $\mathrm{p} * u$ depends only on the values $\partial^{\mu} \widehat{u}(0)$ of $\widehat{u}$ at the origin for $\mu \in \mathbb{N}_{0}^{d}$ with $|\mu|<m$. In other words, if $u, v \in l_{0}\left(\mathbb{Z}^{d}\right)$ satisfy $\widehat{u}(\xi)=\widehat{v}(\xi)+\mathcal{O}\left(\|\xi\|^{m}\right)$ as $\xi \rightarrow 0$, then $\mathrm{p} * u=\mathrm{p} * v$ for all $\mathrm{p} \in \Pi_{m-1}$.

Now we study the action of the transition operator on polynomial spaces.

Theorem 3.2. ([25, Proposition 3.1] and [24, Proposition 4.2]) Let $u \in l_{0}\left(\mathbb{Z}^{d}\right)$ and $\mathrm{M}$ be a $d \times d$ invertible integer matrix. For any given polynomial $\mathrm{p} \in \Pi$,

$$
\mathcal{T}_{u, \mathrm{M}} \mathrm{p}=\mathrm{d}_{\mathrm{M}}\left[\mathrm{p} * u^{\star}\right](\mathrm{M} \cdot)=\mathrm{p}(\mathrm{M} \cdot) * u_{\mathrm{p}}=\sum_{\mu \in \mathbb{N}_{0}^{d}} \frac{\mathrm{d}_{\mathrm{M}}(-i)^{|\mu|}}{\mu !}\left(\partial^{\mu} \mathrm{p}\right)(\mathrm{M} \cdot) \overline{\partial^{\mu} \widehat{u}(0)},
$$

where $u_{\mathrm{p}}$ is any finitely supported sequence on $\mathbb{Z}^{d}$ such that

$$
\widehat{u_{\mathrm{p}}}(\xi)=\mathrm{d}_{\mathrm{M}} \overline{\widehat{u}\left(\left(\mathrm{M}^{\top}\right)^{-1} \xi\right)}+\mathcal{O}\left(\|\xi\|^{\operatorname{deg}(\mathrm{p})+1}\right), \quad \xi \rightarrow \mathbf{0} .
$$

In particular, for any positive integer $m \in \mathbb{N}$, the following statements are equivalent:

(1) $\mathcal{T}_{u, \mathrm{M}} \mathrm{p}=0$ for all polynomial sequences $\mathrm{p} \in \Pi_{m-1}$.

(2) $\mathcal{T}_{u, \mathrm{Mq}}=0$ for all polynomial sequences $\mathrm{q}=(\cdot)^{\mu}$ with $\mu \in \mathbb{N}_{0}^{d}$ and $|\mu|=m-1$.

(3) $\widehat{u}(\xi)=\mathcal{O}\left(\|\xi\|^{m}\right)$ as $\xi \rightarrow \mathbf{0}$, that is, $\partial^{\mu} \widehat{u}(0)=0$ for all $\mu \in \mathbb{N}_{0}^{d}$ with $|\mu|<m$.

Proof. Since $\mathcal{T}_{u, M} \mathrm{P}=\mathrm{d}_{\mathrm{M}}\left[\mathrm{p} * u^{\star}\right](\mathrm{M} \cdot)=\mathrm{d}_{\mathrm{M}} \sum_{\mathrm{k} \in \mathbb{Z}^{d}} \mathrm{p}\left(\mathrm{M}\left(\cdot-\mathrm{M}^{-1} \mathrm{k}\right)\right) \overline{u(-\mathrm{k})}$, by Lemma 3.1 and $\mathrm{p}(\mathrm{M}(\cdot-$ $\left.\left.\mathrm{M}^{-1} \mathrm{k}\right)\right)=\sum_{\mu \in \mathbb{N}_{0}^{d}} \partial^{\mu}(\mathrm{p}(\mathrm{M} \cdot)) \frac{\left(-\mathrm{M}^{-1} \mathrm{k}\right)^{\mu}}{\mu !}$, we see that $\mathcal{T}_{u, \mathrm{M}} \mathrm{P}$ is a polynomial sequence and

$$
\mathrm{d}_{\mathrm{M}}\left[\mathrm{p} * u^{\star}\right](\mathrm{M} \cdot)=\sum_{\mu \in \mathbb{N}_{0}^{d}} \partial^{\mu}(\mathrm{p}(\mathrm{M} \cdot)) \frac{\mathrm{d}_{\mathrm{M}}}{\mu !} \sum_{\mathrm{k} \in \mathbb{Z}^{d}} \overline{u(-\mathrm{k})}\left(-\mathrm{M}^{-1} \mathrm{k}\right)^{\mu}=\sum_{\mu \in \mathbb{N}_{0}^{d}} \frac{(-i)^{|\mu|}}{\mu !} \partial^{\mu}(\mathrm{p}(\mathrm{M} \cdot)) \partial^{\mu} \widehat{u_{\mathrm{p}}}(0),
$$

where we used $\partial^{\mu} \widehat{u_{\mathrm{p}}}(0)=\mathrm{d}_{\mathrm{M}} i^{|\mu|} \sum_{\mathrm{k} \in \mathbb{Z}^{d}} \overline{u(\mathrm{k})}\left(\mathrm{M}^{-1} \mathrm{k}\right)^{\mu}$. Now the identities in (3.10) follow directly from (3.7). The equivalence among items (1)-(3) is a direct consequence of (3.10). 
We say that a filter $u$ has order $m$ vanishing moments if items (1)-(3) in Theorem 3.2 hold. If $u$ has order $m$ but not $m+1$ vanishing moments, we define $\operatorname{vm}(u):=m$ and say that $u$ has the vanishing moments of order $m$. Vanishing moments are important for sparse framelet expansions, since most framelet coefficients are identically zero for any polynomial to certain degree.

We define the coset sequence $u^{[\gamma]}$ (or $\left.u^{[\gamma: \mathrm{M}]}\right)$ of $u=\{u(\mathrm{k})\}_{\mathrm{k} \in \mathbb{Z}^{d}}$ at the coset $\gamma+\mathrm{M} \mathbb{Z}^{d}$ by

$$
\widehat{u^{[\gamma]}}(\xi):=\sum_{\mathbf{k} \in \mathbb{Z}^{d}} u(\gamma+\mathrm{Mk}) e^{-i \mathbf{k} \cdot \xi}, \quad \text { that is, } \quad u^{[\gamma]}=\{u(\gamma+\mathrm{Mk})\}_{\mathbf{k} \in \mathbb{Z}^{d}} .
$$

Now we proceed to investigate the subdivision operator acting on polynomial spaces. In contrast to the case of the transition operator, $\mathcal{S}_{u, \mathrm{MP}}$ is not always a polynomial sequence for an input polynomial sequence $\mathrm{p}$. For example, for $\mathrm{p}=1$ and $u=\delta$, we have $\left[\mathcal{S}_{u, \mathrm{M}} \mathrm{p}\right](\gamma+\mathrm{Mk})=\delta(\gamma) \mathrm{d}_{\mathrm{M}}$ for all $\gamma \in \Gamma_{\mathrm{M}}$ and $\mathrm{k} \in \mathbb{Z}^{d}$. Hence, $\mathcal{S}_{\delta, \mathrm{M}} 1$ is not a polynomial sequence.

Before proceeding further, we need an auxiliary result which is implicitly given in [25].

Lemma 3.3. Let $u=\{u(\mathrm{k})\}_{\mathrm{k} \in \mathbb{Z}^{d}} \in l_{0}\left(\mathbb{Z}^{d}\right)$ and $\mathrm{q} \in \Pi$. Then the following are equivalent:

(i) $\left(\mathrm{q} * u^{[\gamma]}\right)\left(-\mathrm{M}^{-1} \gamma\right)=\sum_{\mathrm{k} \in \mathbb{Z}^{d}} \mathrm{q}\left(-\mathrm{M}^{-1} \gamma-\mathrm{k}\right) u(\gamma+\mathrm{Mk})=\sum_{\mathrm{k} \in \mathbb{Z}^{d}} \mathrm{q}(-\mathrm{k}) u(\mathrm{Mk})=\left(\mathrm{q} * u^{[0]}\right)(0)$ for all $\gamma \in \Gamma_{\mathrm{M}}$.

(ii) $\left.\left[\mathbf{q}(-i \partial)\left(e^{-i \mathrm{M}^{-1} \gamma \cdot \xi} \widehat{u^{[\gamma]}}(\xi)\right)\right]\right|_{\xi=0}=\left.\left[\mathbf{q}(-i \partial) \widehat{u^{[0]}}(\xi)\right]\right|_{\xi=0}$ for all $\gamma \in \Gamma_{\mathbf{M}}$.

(iii) $\left.\left[\mathrm{q}\left(-i \mathrm{M}^{-1} \partial\right) \widehat{u}(\xi)\right]\right|_{\xi=2 \pi \omega}=0$ for all $\omega \in \Omega_{\mathrm{M}} \backslash\{\mathbf{0}\}$.

Proof. The equivalence between (i) and (ii) is a direct consequence of the following identity

$$
\left.\left[\mathbf{q}(-i \partial)\left(e^{-i \mathbf{M}^{-1} \gamma \cdot \xi} \widehat{u[\gamma]}(\xi)\right)\right]\right|_{\xi=0}=\left.\left[\mathbf{q}\left(-\mathbf{M}^{-1} \gamma-i \partial\right) \widehat{u[\gamma]}(\xi)\right]\right|_{\xi=0}=\sum_{\mathbf{k} \in \mathbb{Z}^{d}} \mathbf{q}\left(-\mathbf{M}^{-1} \gamma-\mathbf{k}\right) u(\gamma+\mathbf{M k})
$$

for $\gamma \in \mathbb{Z}^{d}$, where we used (3.4) and (3.7). Note that $\widehat{u}(\xi)=\sum_{\gamma \in \Gamma_{\mathrm{M}}} e^{-i \gamma \cdot \widehat{u^{[\gamma]}}}\left(\mathrm{M}^{\top} \xi\right)$. Therefore, $\widehat{u}\left(\left(\mathrm{M}^{\top}\right)^{-1} \xi+2 \pi \omega\right)=\sum_{\gamma \in \Gamma_{\mathrm{M}}} e^{-i \gamma \cdot 2 \pi \omega} e^{-i \mathrm{M}^{-1} \gamma \cdot \xi \widehat{u} \widehat{[\gamma]}}(\xi)$. Now we see that item (ii) is equivalent to $\left.\left[\mathbf{q}(-i \partial) \widehat{u}\left(\left(\mathrm{M}^{\top}\right)^{-1} \xi+2 \pi \omega\right)\right]\right|_{\xi=0}=0$ for all $\omega \in \Omega_{\mathbf{M}} \backslash\{\mathbf{0}\}$, which is just item (iii).

We have the necessary and sufficient condition for $\mathcal{S}_{u, \mathrm{MP}}$ to be a polynomial sequence.

Theorem 3.4. ([25, Lemma 3.2]) Let $u=\{u(\mathrm{k})\}_{\mathrm{k} \in \mathbb{Z}^{d}} \in l_{0}\left(\mathbb{Z}^{d}\right)$, $\mathrm{M}$ be a $d \times d$ invertible integer matrix, and $\mathrm{p} \in \Pi$ be a polynomial. Then the following statements are equivalent:

(1) $\mathcal{S}_{u, \mathrm{MP}}$ is a polynomial sequence, that is, $\mathcal{S}_{u, \mathrm{MP}} \in \Pi$.

(2) $\sum_{\mathrm{k} \in \mathbb{Z}^{d}}\left(\partial^{\mu} \mathrm{p}\right)\left(-\mathrm{M}^{-1} \gamma-\mathrm{k}\right) u(\gamma+\mathrm{Mk})=\sum_{\mathrm{k} \in \mathbb{Z}^{d}}\left(\partial^{\mu} \mathrm{p}\right)(-\mathrm{k}) u(\mathrm{Mk})$ for all $\mu \in \mathbb{N}_{0}^{d}$ and $\gamma \in \Gamma_{\mathrm{M}}$.

(3) $\left.\left[\left(\partial^{\mu} \mathrm{p}\right)(-i \partial)\left(e^{-i \mathrm{M}^{-1} \gamma \cdot \xi} \widehat{u[\gamma]}(\xi)\right)\right]\right|_{\xi=0}=\left.\left[\left(\partial^{\mu} \mathrm{p}\right)(-i \partial) \widehat{u[0]}(\xi)\right]\right|_{\xi=0}$ for all $\mu \in \mathbb{N}_{0}^{d}$ and $\gamma \in \Gamma_{\mathrm{M}}$.

(4) $\left.\left[\left(\partial^{\mu} \mathrm{p}\right)\left(-\mathrm{M}^{-1} \gamma-i \partial\right) \widehat{u^{[\gamma]}}(\xi)\right]\right|_{\xi=0}=\left.\left[\left(\partial^{\mu} \mathrm{p}\right)(-i \partial) \widehat{u^{[0]}}(\xi)\right]\right|_{\xi=0}$ for all $\mu \in \mathbb{N}_{0}^{d}$ and $\gamma \in \Gamma_{\mathrm{M}}$.

(5) $\left.\left[\left(\partial^{\mu} \mathrm{p}\right)\left(-i \mathrm{M}^{-1} \partial\right) \widehat{u}(\xi)\right]\right|_{\xi=2 \pi \omega}=0$ for all $\mu \in \mathbb{N}_{0}^{d}$ and $\omega \in \Omega_{\mathrm{M}} \backslash\{\mathbf{0}\}$.

Moreover, if any of the above items (1)-(5) holds, then $\operatorname{deg}\left(\mathcal{S}_{u, \mathrm{M}} \mathrm{p}\right) \leqslant \operatorname{deg}(\mathrm{p})$ and

$$
\begin{aligned}
& \mathcal{S}_{u, \mathrm{M}}\left(\partial^{\beta} \mathrm{p}\right)=\left[\left(\partial^{\beta} \mathrm{p}\right)\left(\mathrm{M}^{-1} \cdot\right)\right] * u, \quad \beta \in \mathbb{N}_{0}^{d}, \\
& \mathcal{S}_{u, \mathrm{M}}(\mathrm{p}(\cdot-y))=\mathrm{p}\left(\mathrm{M}^{-1} \cdot-y\right) * u=\left[\mathcal{S}_{u, \mathrm{M}} \mathrm{p}\right](\cdot-\mathrm{M} y), \quad y \in \mathbb{R}^{d} .
\end{aligned}
$$

Proof. By the definition of the subdivision operator $\mathcal{S}_{u, \mathrm{M}}$ in $(2.1)$, for $\mathrm{j}, \gamma \in \mathbb{Z}^{d}$,

$$
\begin{aligned}
{\left[\mathcal{S}_{u, \mathrm{M}} \mathrm{p}\right](\gamma+\mathrm{Mj}) } & =\mathrm{d}_{\mathrm{M}} \sum_{\mathrm{m} \in \mathbb{Z}^{d}} \mathrm{p}(\mathrm{m}) u(\gamma+\mathrm{Mj}-\mathrm{Mm}) \\
& =\mathrm{d}_{\mathrm{M}} \sum_{\mathbf{k} \in \mathbb{Z}^{d}} \mathrm{p}\left(\mathrm{M}^{-1}(\gamma+\mathrm{Mj})-\mathrm{M}^{-1} \gamma-\mathrm{k}\right) u(\gamma+\mathrm{Mk}) .
\end{aligned}
$$


Hence, $\left[\mathcal{S}_{u, \mathrm{M}} \mathrm{P}\right](\gamma+\mathrm{M} \cdot)$ is a polynomial sequence for every $\gamma \in \mathbb{Z}^{d}$. Now it is easy to see that $\mathcal{S}_{u, \mathrm{MP}}$ is a polynomial sequence if and only if $\sum_{\mathrm{k} \in \mathbb{Z}^{d}} \mathrm{p}\left(\cdot-\mathrm{M}^{-1} \gamma-\mathrm{k}\right) u(\gamma+\mathrm{Mk})$ is independent of $\gamma$. Using Taylor expansion of $\mathrm{p}$, we have

$$
\begin{aligned}
\sum_{\mathbf{k} \in \mathbb{Z}^{d}} \mathrm{p}\left(x-\mathrm{M}^{-1} \gamma-\mathrm{k}\right) u(\gamma+\mathrm{Mk}) & =\sum_{\mathrm{k} \in \mathbb{Z}^{d}} \sum_{\mu \in \mathbb{N}_{0}^{d}} \frac{x^{\mu}}{\mu !}\left(\partial^{\mu} \mathrm{p}\right)\left(-\mathrm{M}^{-1} \gamma-\mathrm{k}\right) u(\gamma+\mathrm{Mk}) \\
& =\sum_{\mu \in \mathbb{N}_{0}^{d}} \frac{x^{\mu}}{\mu !} \sum_{\mathrm{k} \in \mathbb{Z}^{d}}\left(\partial^{\mu} \mathrm{p}\right)\left(-\mathrm{M}^{-1} \gamma-\mathrm{k}\right) u(\gamma+\mathrm{Mk}) .
\end{aligned}
$$

Hence, $\sum_{\mathrm{k} \in \mathbb{Z}^{d}} \mathrm{p}\left(\cdot-\mathrm{M}^{-1} \gamma-\mathrm{k}\right) u(\gamma+\mathrm{Mk})$ is independent of $\gamma$ if and only if all $\sum_{\mathbf{k} \in \mathbb{Z}^{d}}\left(\partial^{\mu} \mathrm{p}\right)\left(-\mathrm{M}^{-1} \gamma-\right.$ k) $u(\gamma+\mathrm{Mk})$ are independent of $\gamma$, which are obviously equivalent to the conditions in item (2). Thus, we proved $(1) \Longleftrightarrow(2)$. Moreover, when $\mathcal{S}_{u, \mathrm{MP}} \in \Pi$, the above argument also yields

$$
\mathcal{S}_{u, \mathrm{M}} \mathrm{p}=\mathrm{d}_{\mathrm{M}} \sum_{\mathrm{k} \in \mathbb{Z}^{d}} \mathrm{p}\left(\mathrm{M}^{-1} \cdot-\mathrm{M}^{-1} \gamma-\mathrm{k}\right) u(\gamma+\mathrm{Mk})=\sum_{\mathrm{k} \in \mathbb{Z}^{d}} \mathrm{p}\left(\mathrm{M}^{-1}(\cdot-\mathrm{k})\right) u(\mathrm{k}), \quad \forall \gamma \in \mathbb{Z}^{d},
$$

from which we see that (3.13) holds. The equivalence among (2)-(5) follows from Lemma 3.3.

For subdivision operator acting on polynomial spaces, we now have the following result.

Theorem 3.5. ([25, Proposition 3.3] and [24, Proposition 4.3]) Let $u=\{u(\mathrm{k})\}_{\mathrm{k} \in \mathbb{Z}^{d}} \in l_{0}\left(\mathbb{Z}^{d}\right)$ and $\mathrm{M}$ be a $d \times d$ invertible integer matrix. For any positive integer $m \in \mathbb{N}$, the following are equivalent:

(1) $\mathcal{S}_{u, \mathrm{M}} \Pi_{m-1} \subseteq \Pi$, or $\mathcal{S}_{u, \mathrm{M}} \Pi_{m-1} \subseteq \Pi_{m-1}$.

(2) $\mathcal{S}_{u, \mathrm{Mq}} \in \Pi$ for all polynomials $\mathrm{q}=(\cdot)^{\mu}$ with $\mu \in \mathbb{N}_{0}^{d}$ and $|\mu|=m-1$.

(3) $\partial^{\mu} \widehat{u}(2 \pi \omega)=0$ for all $\mu \in \mathbb{N}_{0}^{d}$ with $|\mu|<m$ and for all $\omega \in \Omega_{\mathrm{M}} \backslash\{\mathbf{0}\}$; in other words,

$$
\widehat{u}(\xi+2 \pi \omega)=\mathcal{O}\left(\|\xi\|^{m}\right), \quad \xi \rightarrow 0, \quad \forall \omega \in \Omega_{\mathrm{M}} \backslash\{\mathbf{0}\} .
$$

(4) $e^{-i \mathrm{M}^{-1} \gamma \cdot \xi} \widehat{u^{[\gamma]}}(\xi)=\widehat{u^{[0]}}(\xi)+\mathcal{O}\left(\|\xi\|^{m}\right)$ as $\xi \rightarrow 0$ for all $\gamma \in \Gamma_{\mathrm{M}}$, or its equivalent form:

$$
\sum_{\mathbf{k} \in \mathbb{Z}^{d}} u(\gamma+\mathrm{Mk})(\gamma+\mathrm{Mk})^{\mu}=\sum_{\mathbf{k} \in \mathbb{Z}^{d}} u(\mathrm{Mk})(\mathrm{Mk})^{\mu}, \quad \forall \mu \in \mathbb{N}_{0}^{d},|\mu|<m .
$$

If (3.15) holds, then for all $\mathrm{p} \in \Pi_{m-1}$ and $v \in l_{0}\left(\mathbb{Z}^{d}\right), \mathcal{S}_{u, \mathrm{M}}(\mathrm{p} * v)=\mathrm{p}\left(\mathrm{M}^{-1} \cdot\right) *\left[\mathcal{S}_{u, \mathrm{M} v}\right]$.

Proof. (1) $\Longrightarrow(2)$ is obvious. By Theorem 3.4, if $\mathcal{S}_{u, \mathrm{M}} \mathrm{p} \in \Pi$, then $\mathcal{S}_{u, \mathrm{M}}\left(\partial^{\mu} \mathrm{p}\right) \in \Pi$ for all $\mu \in \mathbb{N}_{0}^{d}$. The equivalence between (1)-(4) now follows from Theorem 3.4.

We say that a filter $u$ has order $m$ sum rules if any of items (1)-(4) in Theorem 3.5 is satisfied. If $u$ has order $m$ but not $m+1$ sum rules, we define $\operatorname{sr}(u):=m$ and we say that $u$ has the sum rules of order $m$. The action of the subdivision operator on polynomial sequences has been initially (but implicitly) investigated in [16, Section 2] and [19, Section 2].

\section{Stability of Multilevel Discrete Framelet Transforms}

One of the key features of a discrete framelet transform is its ability to extract the multiscale structure embedded in signals. For this purpose, a multilevel discrete framelet transform is often used in applications by recursively performing one-level discrete framelet transforms on selected sequences of framelet coefficients at the immediate higher scale level. In this section, we recall a (standard) multilevel discrete framelet transform, introduce the notion of its stability in the sequence space $l_{2}\left(\mathbb{Z}^{d}\right)$, and then study its stability and discrete affine systems in $l_{2}\left(\mathbb{Z}^{d}\right)$. 


\subsection{Multilevel discrete framelet transforms}

In the following, we describe a commonly used standard multilevel discrete framelet transform by recursively performing one-level discrete framelet transforms on only one selected sequence of framelet coefficients. Of course, one can select several or even all the sequences of framelet coefficients for further decomposition, but we have more or less the same algorithm. The framelet coefficients and their associated filters in such selected sequences for further decomposition are called parent (or low-pass) framelet coefficients and parent (or low-pass) filters or masks (since they are often low-pass filters), respectively. In this paper, we use $a$ or its indexed version to denote a low-pass (or parent) filter and use $v$ or its indexed version to denote low-pass (or parent) framelet coefficients. The framelet coefficients and their associated filters in other sequences, which are not selected for further decomposition, are called child (or high-pass) framelet coefficients and child (or high-pass) filters (since they are often high-pass filters), respectively. In this paper, we use $b$ or its indexed version to denote a high-pass (or child) filter and use $w$ or its indexed version to denote high-pass (or child) framelet coefficients.

Let $\tilde{a}$ be a dual low-pass filter and $\tilde{b}_{1}, \ldots, \tilde{b}_{s}$ be dual high-pass filters for decomposition. For a positive integer $J$, a J-level discrete framelet decomposition is given by

$$
v_{j-1}:=\mathrm{d}_{\mathrm{M}}^{-1 / 2} \mathcal{T}_{\tilde{a}, \mathrm{M}} v_{j}, \quad w_{j-1 ; \ell}:=\mathrm{d}_{\mathrm{M}}^{-1 / 2} \mathcal{T}_{\tilde{b}_{\ell}, \mathrm{M}} v_{j}, \quad \ell=1, \ldots, s, \quad j=J, \ldots, 1,
$$

where $v_{J}: \mathbb{Z}^{d} \rightarrow \mathbb{C}$ is an input signal. After a $J$-level discrete framelet decomposition, the original input signal $v_{J}$ is decomposed into one sequence $v_{0}$ of low-pass framelet coefficients and $s J$ sequences $w_{j ; \ell}$ of high-pass framelet coefficients for $\ell=1, \ldots, s$ and $j=0, \ldots, J-1$. For various purposes, such framelet coefficients are often processed by thresholding or quantization.

Let $a$ be a primal low-pass filter and $b_{1}, \ldots, b_{s}$ be primal high-pass filters for reconstruction. Now $a$ $J$-level discrete framelet reconstruction is

$$
\stackrel{\circ}{v}_{j}:=\mathrm{d}_{\mathrm{M}}^{-1 / 2} \mathcal{S}_{a, \mathrm{M}} \stackrel{\circ}{j-1}_{j}+\mathrm{d}_{\mathrm{M}}^{-1 / 2} \sum_{\ell=1}^{s} \mathcal{S}_{b_{\ell}, \mathrm{M}} \stackrel{\circ}{w}_{j-1 ; \ell}, \quad j=1, \ldots, J .
$$

For analysis of a multilevel discrete framelet transform, it is convenient to rewrite the $J$-level discrete framelet decomposition employing the filter bank $\left\{\tilde{a} ; \tilde{b}_{1}, \ldots, \tilde{b}_{s}\right\}$ by using a J-level decomposition operator $\widetilde{\mathcal{W}}_{J}: l(\mathbb{Z}) \rightarrow(l(\mathbb{Z}))^{1 \times(s J+1)}$ as follows:

$$
\widetilde{\mathcal{W}}_{J} v_{J}:=\left(w_{J-1 ; 1}, \ldots, w_{J-1 ; s}, \ldots, w_{0 ; 1}, \ldots, w_{0 ; s}, v_{0}\right)
$$

where $w_{j-1 ; \ell}$ and $v_{0}$ are defined in (4.1). Similarly, a J-level discrete reconstruction operator $\mathcal{V}_{J}$ : $(l(\mathbb{Z}))^{1 \times(s J+1)} \rightarrow l(\mathbb{Z})$ employing the filter bank $\left\{a ; b_{1}, \ldots, b_{s}\right\}$ is defined by

$$
\mathcal{V}_{J}\left(\stackrel{\circ}{w}_{J-1 ; 1}, \ldots, \stackrel{\circ}{w}_{J-1 ; s}, \ldots, \stackrel{\circ}{w}_{0 ; 1}, \ldots, \stackrel{\circ}{w}_{0 ; s}, \stackrel{\circ}{0}_{0}\right)=\stackrel{\circ}{v}_{J}
$$

where $\dot{v}_{J}$ is computed via the recursive formulas in (4.2). Due to the recursive cascade structure of the operators $\widetilde{\mathcal{W}}_{J}$ and $\mathcal{V}_{J}$ in (4.1) and (4.2), a multilevel discrete framelet transform is often called a fast framelet transform. A fast framelet transform with $s=\mathrm{d}_{\mathrm{M}}-1$ is further called a fast wavelet transform. We shall denote a $J$-level discrete framelet decomposition operator employing the filter bank $\left\{a ; b_{1}, \ldots, b_{s}\right\}$ by $\mathcal{W}_{J}$ and a $J$-level discrete framelet reconstruction operator employing the filter bank $\left\{\tilde{a} ; \tilde{b}_{1}, \ldots, \tilde{b}_{s}\right\}$ by $\widetilde{\mathcal{V}}_{J}$.

We say that a $J$-level discrete framelet transform has the perfect reconstruction property if $\mathcal{V}_{J} \widetilde{\mathcal{W}}_{J} v_{J}=$ $v_{J}$, that is, the reconstructed signal $\stackrel{\circ}{v}_{J}$ is the same as the original input signal $v_{J}$ if $\dot{v}_{0}=v_{0}$ and $\stackrel{\circ}{j ; \ell}_{j}=w_{j, \ell}$ for all $\ell=1, \ldots, s$ and $j=0, \ldots, J-1$. For example, by Theorem 2.1, a $J$-level discrete framelet transform employing a filter bank $\left(\left\{\tilde{a} ; \tilde{b}_{1}, \ldots, \tilde{b}_{s}\right\},\left\{a ; b_{1}, \ldots, b_{s}\right\}\right)$ has the perfect reconstruction property if for all 
$\xi \in \mathbb{R}^{d}$,

$$
\begin{gathered}
{\left[\begin{array}{cccc}
\widehat{\tilde{a}}\left(\xi+2 \pi \omega_{1}\right) & \widehat{\tilde{b}_{1}}\left(\xi+2 \pi \omega_{1}\right) & \cdots & \widehat{\tilde{b}}_{s}\left(\xi+2 \pi \omega_{1}\right) \\
\vdots & \vdots & \ddots & \vdots \\
\widehat{\tilde{a}}\left(\xi+2 \pi \omega_{\mathrm{d}_{\mathrm{M}}}\right) & \widetilde{b}_{1}\left(\xi+2 \pi \omega_{\mathrm{d}_{\mathrm{M}}}\right) & \cdots & \widehat{\vec{b}}_{s}\left(\xi+2 \pi \omega_{\mathrm{d}_{\mathrm{M}}}\right)
\end{array}\right]} \\
{\left[\begin{array}{cccc}
\widehat{a}\left(\xi+2 \pi \omega_{1}\right) & \widehat{b_{1}}\left(\xi+2 \pi \omega_{1}\right) & \cdots & \widehat{b_{s}}\left(\xi+2 \pi \omega_{1}\right) \\
\vdots & \vdots & \ddots & \vdots \\
\widehat{a}\left(\xi+2 \pi \omega_{\mathrm{d}_{\mathrm{M}}}\right) & \widehat{b_{1}}\left(\xi+2 \pi \omega_{\mathrm{d}_{\mathrm{M}}}\right) & \cdots & \widehat{b_{s}}\left(\xi+2 \pi \omega_{\mathrm{d}_{\mathrm{M}}}\right)
\end{array}\right]^{\star}=I_{\mathrm{d}_{\mathrm{M}}} .}
\end{gathered}
$$

That is, $\left(\left\{\tilde{a} ; \tilde{b}_{1}, \ldots, \tilde{b}_{s}\right\},\left\{a ; b_{1}, \ldots, b_{s}\right\}\right)$ is a dual M-framelet filter bank. Note that $\mathcal{W}_{J}^{\star}=\mathcal{V}_{J}, \widetilde{\mathcal{W}}_{J}^{\star}=\widetilde{\mathcal{V}}_{J}$. Therefore, $\mathcal{V}_{J} \widetilde{\mathcal{W}}_{J} v=v$ for all $v \in l\left(\mathbb{Z}^{d}\right)$ if and only if $\widetilde{\mathcal{V}}_{J} \mathcal{W}_{J} v=v$ for all $v \in l\left(\mathbb{Z}^{d}\right)$.

\subsection{Stability of multilevel discrete framelet transforms}

Beyond the perfect reconstruction property and sparsity that we have discussed in Sections 2 and 3 , another fundamental property is stability. We say that a filter bank $\left\{a ; b_{1}, \ldots, b_{s}\right\}$ has stability in $l_{2}\left(\mathbb{Z}^{d}\right)$ if there exist positive constants $C_{1}$ and $C_{2}$ such that

$$
C_{1}\|v\|_{l_{2}\left(\mathbb{Z}^{d}\right)} \leqslant\left\|\mathcal{W}_{J} v\right\|_{\left(l_{2}\left(\mathbb{Z}^{d}\right)\right)^{1 \times(s J+1)}} \leqslant C_{2}\|v\|_{l_{2}\left(\mathbb{Z}^{d}\right)}, \quad \forall v \in l_{2}\left(\mathbb{Z}^{d}\right), J \in \mathbb{N} .
$$

A filter bank $\left\{a ; b_{1}, \ldots, b_{s}\right\}$ having stability in $l_{2}\left(\mathbb{Z}^{d}\right)$ is also called a framelet filter bank (with stability) in $l_{2}\left(\mathbb{Z}^{d}\right)$. By (4.6), the $l_{2}$-norm of framelet coefficients provides an equivalent norm for the sequence space $l_{2}\left(\mathbb{Z}^{d}\right)$. We say that a multilevel discrete framelet transform employing a dual $\mathrm{M}$-framelet filter bank $\left(\left\{\tilde{a} ; \tilde{b}_{1}, \ldots, \tilde{b}_{s}\right\},\left\{a ; b_{1}, \ldots, b_{s}\right\}\right)$ has stability in the space $l_{2}\left(\mathbb{Z}^{d}\right)$, or simply a dual M-framelet filter bank $\left(\left\{\tilde{a} ; \tilde{b}_{1}, \ldots, \tilde{b}_{s}\right\},\left\{a ; b_{1}, \ldots, b_{s}\right\}\right)$ has stability in $l_{2}\left(\mathbb{Z}^{d}\right)$, if both filter banks $\left\{\tilde{a} ; \tilde{b}_{1}, \ldots, \tilde{b}_{s}\right\}$ and $\left\{a ; b_{1}, \ldots, b_{s}\right\}$ have stability in the space $l_{2}\left(\mathbb{Z}^{d}\right)$.

We now have the following result on the stability of a multilevel discrete framelet transform.

Theorem 4.1. Let $\left(\left\{\tilde{a} ; \tilde{b}_{1}, \ldots, \tilde{b}_{s}\right\},\left\{a ; b_{1}, \ldots, b_{s}\right\}\right)$ be a dual $\mathrm{M}$-framelet filter bank. Let $\mathcal{W}_{J}, \widetilde{\mathcal{W}}_{J}$ be its associated $J$-level discrete framelet decomposition operators and $\mathcal{V}_{J}, \widetilde{\mathcal{V}}_{J}$ be its associated J-level discrete framelet reconstruction operators. Then the following statements are equivalent:

(i) The dual M-framelet filter bank $\left(\left\{\tilde{a} ; \tilde{b}_{1}, \ldots, \tilde{b}_{s}\right\},\left\{a ; b_{1}, \ldots, b_{s}\right\}\right)$ has stability in the space $l_{2}\left(\mathbb{Z}^{d}\right)$, that is, there exist positive constants $C_{1}$ and $C_{2}$ such that (4.6) holds and

$$
C_{2}^{-1}\|v\|_{l_{2}\left(\mathbb{Z}^{d}\right)} \leqslant\left\|\widetilde{\mathcal{W}}_{J} v\right\|_{\left(l_{2}\left(\mathbb{Z}^{d}\right)\right)^{1 \times(s J+1)}} \leqslant C_{1}^{-1}\|v\|_{l_{2}\left(\mathbb{Z}^{d}\right)}, \quad \forall v \in l_{2}\left(\mathbb{Z}^{d}\right), J \in \mathbb{N} .
$$

(ii) There exist positive constants $C_{1}$ and $C_{2}$ such that for all $v \in l_{2}\left(\mathbb{Z}^{d}\right)$ and $J \in \mathbb{N}$,

$$
\left\|\mathcal{W}_{J} v\right\|_{\left(l_{2}\left(\mathbb{Z}^{d}\right)\right)^{1 \times(s J+1)}} \leqslant C_{2}\|v\|_{l_{2}\left(\mathbb{Z}^{d}\right)} \quad \text { and } \quad\left\|\widetilde{\mathcal{W}}_{J} v\right\|_{\left(l_{2}\left(\mathbb{Z}^{d}\right)\right)^{1 \times(s J+1)}} \leqslant C_{1}^{-1}\|v\|_{l_{2}\left(\mathbb{Z}^{d}\right)} .
$$

(iii) There exist $C_{1}>0$ and $C_{2}>0$ such that for all $\boldsymbol{w} \in\left(l_{2}\left(\mathbb{Z}^{d}\right)\right)^{1 \times(s J+1)}$ and for all $J \in \mathbb{N}$,

$$
\left\|\mathcal{V}_{J} \boldsymbol{w}\right\|_{l_{2}\left(\mathbb{Z}^{d}\right)} \leqslant C_{2}\|\boldsymbol{w}\|_{\left(l_{2}\left(\mathbb{Z}^{d}\right)\right)^{1 \times(s J+1)}} \quad \text { and } \quad\left\|\widetilde{\mathcal{V}}_{J} \boldsymbol{w}\right\|_{l_{2}\left(\mathbb{Z}^{d}\right)} \leqslant C_{1}^{-1}\|\boldsymbol{w}\|_{\left(l_{2}\left(\mathbb{Z}^{d}\right)\right)^{1 \times(s J+1)}} .
$$

(iv) There exist $C_{1}, C_{2}>0$ such that for all $v \in l_{2}\left(\mathbb{Z}^{d}\right), \boldsymbol{w} \in\left(l_{2}\left(\mathbb{Z}^{d}\right)\right)^{1 \times(s J+1)}$, and for all $J \in \mathbb{N}$,

$$
\left\|\mathcal{V}_{J} \boldsymbol{w}\right\|_{l_{2}\left(\mathbb{Z}^{d}\right)} \leqslant C_{2}\|\boldsymbol{w}\|_{\left(l_{2}\left(\mathbb{Z}^{d}\right)\right)^{1 \times(s J+1)}} \quad \text { and } \quad\left\|\widetilde{\mathcal{W}}_{J} v\right\|_{\left(l_{2}\left(\mathbb{Z}^{d}\right)\right)^{1 \times(s J+1)}} \leqslant C_{1}^{-1}\|v\|_{l_{2}\left(\mathbb{Z}^{d}\right)} .
$$

(v) There exist $C_{1}, C_{2}>0$ such that for all $v \in l_{2}\left(\mathbb{Z}^{d}\right), \boldsymbol{w} \in\left(l_{2}\left(\mathbb{Z}^{d}\right)\right)^{1 \times(s J+1)}$, and for all $J \in \mathbb{N}$,

$$
\left\|\mathcal{W}_{J} v\right\|_{\left(l_{2}\left(\mathbb{Z}^{d}\right)\right)^{1 \times(s J+1)}} \leqslant C_{2}\|v\|_{l_{2}\left(\mathbb{Z}^{d}\right)} \quad \text { and } \quad\left\|\widetilde{\mathcal{V}}_{J} \boldsymbol{w}\right\|_{l_{2}\left(\mathbb{Z}^{d}\right)} \leqslant C_{1}^{-1}\|\boldsymbol{w}\|_{\left(l_{2}\left(\mathbb{Z}^{d}\right)\right)^{1 \times(s J+1)}} .
$$


If in addition $s=\mathrm{d}_{\mathrm{M}}-1$, any of the above statements is equivalent to

(vi) There exist positive constants $C_{1}$ and $C_{2}$ such that for all $\boldsymbol{w} \in\left(l_{2}\left(\mathbb{Z}^{d}\right)\right)^{1 \times(s J+1)}$ and $J \in \mathbb{N}$,

$$
C_{1}\|\boldsymbol{w}\|_{\left(l_{2}\left(\mathbb{Z}^{d}\right)\right)^{1 \times(s J+1)}} \leqslant\left\|\mathcal{V}_{J} \boldsymbol{w}\right\|_{l_{2}\left(\mathbb{Z}^{d}\right)} \leqslant C_{2}\|\boldsymbol{w}\|_{\left(l_{2}\left(\mathbb{Z}^{d}\right)\right)^{1 \times(s J+1)}}
$$

and

$$
C_{2}^{-1}\|\boldsymbol{w}\|_{\left(l_{2}\left(\mathbb{Z}^{d}\right)\right)^{1 \times(s J+1)}} \leqslant\left\|\widetilde{\mathcal{V}}_{J} \boldsymbol{w}\right\|_{l_{2}\left(\mathbb{Z}^{d}\right)} \leqslant C_{1}^{-1}\|\boldsymbol{w}\|_{\left(l_{2}\left(\mathbb{Z}^{d}\right)\right)^{1 \times(s J+1)}} .
$$

Proof. (i) $\Longrightarrow$ (ii) is trivial. Note that $\mathcal{V}_{J}=\mathcal{W}_{J}^{\star}$ and $\widetilde{\mathcal{V}}_{J}=\widetilde{\mathcal{W}}_{J}^{\star}$. It follows from the well-known identities $\left\|\mathcal{W}_{J}^{\star}\right\|=\left\|\mathcal{W}_{J}\right\|$ and $\left\|\widetilde{\mathcal{W}}_{J}^{\star}\right\|=\left\|\widetilde{\mathcal{W}}_{J}\right\|$ that (ii) $\Longleftrightarrow$ (iii) $\Longleftrightarrow$ (iv) $\Longleftrightarrow(\mathrm{v}$ ), where $\|\cdot\|$ here refers to the operator norm in the space $l_{2}\left(\mathbb{Z}^{d}\right)$.

We now prove that (ii) and (iii) together imply (i). Since $\left(\left\{\tilde{a} ; \tilde{b}_{1}, \ldots, \tilde{b}_{s}\right\},\left\{a ; b_{1}, \ldots, b_{s}\right\}\right)$ is a dual Mframelet filter bank, we have the perfect reconstruction property $\widetilde{\mathcal{V}}_{J} \mathcal{W}_{J} v=\mathcal{V}_{J} \widetilde{\mathcal{W}}_{J} v=v$ for all $v \in l_{2}\left(\mathbb{Z}^{d}\right)$. By (4.8) and (4.9), we have

$$
\|v\|_{l_{2}\left(\mathbb{Z}^{d}\right)}=\left\|\widetilde{\mathcal{V}}_{J} \mathcal{W}_{J} v\right\|_{l_{2}\left(\mathbb{Z}^{d}\right)} \leqslant C_{1}^{-1}\left\|\mathcal{W}_{J} v\right\|_{\left(l_{2}\left(\mathbb{Z}^{d}\right)\right)^{1 \times(s J+1)}}
$$

from which and (4.8) we see that (4.6) holds. (4.7) can be proved similarly.

Since $v=\mathcal{V}_{J} \widetilde{\mathcal{W}}_{J} v$ for all $v \in l_{2}\left(\mathbb{Z}^{d}\right)$, replacing $\|v\|_{l_{2}\left(\mathbb{Z}^{d}\right)}$ in $(4.7)$ by $\left\|\mathcal{V}_{J} \widetilde{\mathcal{W}}_{J} v\right\|_{l_{2}\left(\mathbb{Z}^{d}\right)}$, we deduce

$$
C_{1}\left\|\widetilde{\mathcal{W}}_{J} v\right\|_{\left(l_{2}\left(\mathbb{Z}^{d}\right)\right)^{1 \times(s J+1)}} \leqslant\left\|\mathcal{V}_{J} \widetilde{\mathcal{W}}_{J} v\right\|_{l_{2}\left(\mathbb{Z}^{d}\right)} \leqslant C_{2}\left\|\widetilde{\mathcal{W}}_{J} v\right\|_{\left(l_{2}\left(\mathbb{Z}^{d}\right)\right)^{1 \times(s J+1)}}
$$

for all $v \in l_{2}\left(\mathbb{Z}^{d}\right)$ and $J \in \mathbb{N}$. If $s=\mathrm{d}_{\mathrm{M}}-1$, then $\widetilde{\mathcal{W}}_{J}$ is onto, and therefore, (4.12) follows directly from (4.14). (4.13) can be proved similarly.

For $\mathcal{V}_{J}$ and $\widetilde{\mathcal{V}}_{J}$, generally we can only have (4.14) and its duality part by replacing $\widetilde{\mathcal{W}}_{J}$ and $\mathcal{V}_{J}$ in (4.14) with $\mathcal{W}_{J}$ and $\widetilde{\mathcal{V}}_{J}$, respectively. For $s \geqslant \mathrm{~d}_{\mathrm{M}}$, both (4.12) and (4.13) cannot hold, since by Proposition 2.2, there exists $\boldsymbol{w} \not \equiv 0$ in the space $l_{0}\left(\mathbb{Z}^{d}\right)$ such that $\mathcal{V}_{J} \boldsymbol{w}=0$. Obviously, (4.10) implies that a small change of an input data $v$ induces a small change of all framelet coefficients, and a small perturbation of all framelet coefficients results in a small perturbation of a reconstructed signal. The notion of stability of a multilevel discrete framelet transform can be extended to other (weighted) sequence spaces and we shall address such issue elsewhere. Here we only provide some connections between stability of a multilevel discrete framelet transform and a refinable function.

Proposition 4.2. Let $\left\{a ; b_{1}, \ldots, b_{s}\right\}$ be a filter bank such that $\widehat{a}(\mathbf{0})=1$ and there exists a positive constant $C$ such that

$$
\left\|\mathcal{W}_{J} v\right\|_{\left(l_{2}\left(\mathbb{Z}^{d}\right)\right)^{1 \times(s J+1)}}^{2} \leqslant C\|v\|_{l_{2}\left(\mathbb{Z}^{d}\right)}^{2} \quad \forall v \in l_{2}\left(\mathbb{Z}^{d}\right), J \in \mathbb{N}
$$

Let $\mathrm{M}$ be a $d \times d$ expansive integer matrix, that is, all its eigenvalues are greater than one in modulus. Define a frequency-based refinable function $\varphi$ by

$$
\varphi(\xi):=\prod_{j=1}^{\infty} \widehat{a}\left(\left(\mathrm{M}^{\top}\right)^{-j} \xi\right), \quad \xi \in \mathbb{R}^{d} .
$$

Then $\varphi \in L_{2}\left(\mathbb{R}^{d}\right)$ with $\|\varphi\|_{L_{2}\left(\mathbb{R}^{d}\right)}^{2} \leqslant(2 \pi)^{d} C$.

Proof. Since $\mathcal{V}_{J}=\mathcal{W}_{J}^{\star}$, by $(4.15)$, we have

$$
\left\|\mathcal{V}_{J} \boldsymbol{w}\right\|_{l_{2}\left(\mathbb{Z}^{d}\right)}^{2} \leqslant C\|\boldsymbol{w}\|_{\left(l_{2}\left(\mathbb{Z}^{d}\right)\right)^{1 \times(s J+1)}}^{2} \quad \forall \boldsymbol{w} \in\left(l_{2}\left(\mathbb{Z}^{d}\right)\right)^{1 \times(s J+1)}, J \in \mathbb{N} .
$$


Take $\boldsymbol{w}_{0}=(0, \ldots, 0, \delta)$, that is, $v_{0}=\delta$ and all high-pass framelet coefficients vanish. Then $\mathcal{V}_{J} \boldsymbol{w}_{0}=$ $\mathrm{d}_{\mathrm{M}}^{-J / 2} \mathcal{S}_{a, \mathrm{M}}^{J} \delta$. Since the Fourier series of $\mathcal{S}_{a, \mathrm{M}}^{J} \delta$ is $\mathrm{d}_{\mathrm{M}}^{J} \prod_{j=0}^{J-1} \widehat{a}\left(\left(\mathrm{M}^{\mathrm{T}}\right)^{j} \xi\right)$, we have

$$
\begin{aligned}
\left\|\mathcal{V}_{J} \boldsymbol{w}_{0}\right\|_{l_{2}\left(\mathbb{Z}^{d}\right)}^{2}=\left\|\mathrm{d}_{\mathrm{M}}^{-J / 2} \mathcal{S}_{a, \mathrm{M}}^{J} \delta\right\|_{l_{2}\left(\mathbb{Z}^{d}\right)}^{2} & =\mathrm{d}_{\mathrm{M}}^{-J}\left\|\mathcal{S}_{a, \mathrm{M}}^{J} \delta\right\|_{l_{2}\left(\mathbb{Z}^{d}\right)}^{2}=\frac{\mathrm{d}_{\mathrm{M}}^{J}}{(2 \pi)^{d}} \int_{[-\pi, \pi)^{d}} \prod_{j=0}^{J-1}\left|\widehat{a}\left(\left(\mathrm{M}^{\top}\right)^{j} \xi\right)\right|^{2} d \xi \\
& =\frac{1}{(2 \pi)^{d}} \int_{\mathbb{R}^{d}} \chi_{\left(\mathrm{M}^{\top}\right)^{J}[-\pi, \pi)^{d}}(\xi) \prod_{j=1}^{J}\left|\widehat{a}\left(\left(\mathrm{M}^{\top}\right)^{-j} \xi\right)\right|^{2} d \xi
\end{aligned}
$$

It now follows from $\left\|\mathcal{V}_{J} \boldsymbol{w}_{0}\right\|_{l_{2}\left(\mathbb{Z}^{d}\right)}^{2} \leqslant C\left\|\boldsymbol{w}_{0}\right\|_{l_{2}\left(\mathbb{Z}^{d}\right)}^{2}=C$ that

$$
\frac{1}{(2 \pi)^{d}} \int_{\mathbb{R}^{d}} \chi_{\left(\mathrm{M}^{\top}\right)^{J}[-\pi, \pi)^{d}}(\xi) \prod_{j=1}^{J}\left|\widehat{a}\left(\left(\mathrm{M}^{\top}\right)^{-j} \xi\right)\right|^{2} d \xi=\left\|\mathrm{d}_{\mathrm{M}}^{-J / 2} \mathcal{S}_{a, \mathrm{M}}^{J} \delta\right\|_{l_{2}\left(\mathbb{Z}^{d}\right)}^{2}=\left\|\mathcal{V}_{J} \boldsymbol{w}_{0}\right\|_{l_{2}\left(\mathbb{Z}^{d}\right)}^{2} \leqslant C .
$$

Since $M$ is expansive, we have $\lim _{J \rightarrow \infty} \chi_{\left(M^{\top}\right)^{J}[-\pi, \pi)^{d}}(\xi) \prod_{j=1}^{J}\left|\widehat{a}\left(\left(M^{\top}\right)^{-j} \xi\right)\right|^{2}=|\varphi(\xi)|^{2}$ for all $\xi \in \mathbb{R}^{d}$. Applying Fatou's lemma, we have

$$
\frac{1}{(2 \pi)^{d}} \int_{\mathbb{R}^{d}}|\varphi(\xi)|^{2} d \xi \leqslant \liminf _{J \rightarrow \infty} \frac{1}{(2 \pi)^{d}} \int_{\mathbb{R}^{d}} \chi_{\left(\mathrm{M}^{\top}\right)^{J}[-\pi, \pi)^{d}}(\xi) \prod_{j=1}^{J}\left|\widehat{a}\left(\left(\mathrm{M}^{\top}\right)^{-j} \xi\right)\right|^{2} d \xi \leqslant C .
$$

Hence, $\boldsymbol{\varphi} \in L_{2}\left(\mathbb{R}^{d}\right)$ and $\|\varphi\|_{L_{2}\left(\mathbb{R}^{d}\right)}^{2} \leqslant(2 \pi)^{d} C$.

\subsection{Discrete affine systems for $l_{2}\left(\mathbb{Z}^{d}\right)$}

In this section, we explain the role played by the dilation matrix $M$ in a multilevel discrete framelet transform. Then we give the definition of discrete affine systems for $l_{2}\left(\mathbb{Z}^{d}\right)$. In other words, we introduce the notion of discrete framelets and discrete wavelets for $l_{2}\left(\mathbb{Z}^{d}\right)$ in this section.

To implement the subdivision and transition operators using the convolution operation, we need the upsampling and downsampling operators on sequences in $l\left(\mathbb{Z}^{d}\right)$. The upsampling operator $\uparrow \mathrm{M}: l\left(\mathbb{Z}^{d}\right) \rightarrow$ $l\left(\mathbb{Z}^{d}\right)$ and the downsampling (or decimation) operator $\downarrow \mathrm{M}: l\left(\mathbb{Z}^{d}\right) \rightarrow l\left(\mathbb{Z}^{d}\right)$ with a $d \times d$ sampling matrix $\mathrm{M}$ are defined to be

$$
[v \uparrow \mathrm{M}](\mathrm{n}):=\left\{\begin{array}{ll}
v\left(\mathrm{M}^{-1} \mathrm{n}\right), & \text { if } \mathrm{n} \in \mathrm{M}^{d} ; \\
0, & \text { otherwise, }
\end{array} \quad \text { and } \quad[v \downarrow \mathrm{M}](\mathrm{n}):=v(\mathrm{Mn}), \quad \mathrm{n} \in \mathbb{Z}^{d} .\right.
$$

It is convenient to use the notation $v\left(\mathrm{M}^{-1} \cdot\right)$ for $v \uparrow \mathrm{M}$ and $v(\mathrm{M} \cdot)$ for $v \downarrow \mathrm{M}$. Now the subdivision operator $\mathcal{S}_{u, \mathrm{M}}$ in (2.1) and the transition operator $\mathcal{T}_{u, \mathrm{M}}$ in (2.2) can be equivalently expressed as follows:

$$
\mathcal{S}_{u, \mathrm{M}} v=\mathrm{d}_{\mathrm{M}} u *(v \uparrow \mathrm{M}) \quad \text { and } \quad \mathcal{T}_{u, \mathrm{M}} v=\mathrm{d}_{\mathrm{M}}\left(u^{\star} * v\right) \downarrow \mathrm{M} .
$$

According to (4.19), the subdivision and transition operators differ to the convolution operation only in the use of the upsampling and downsampling operators with the sampling matrix $\mathrm{M}$, which plays a crucial role in a multilevel discrete framelet transform to extract multiscale structure embedded in a signal. To understand this point well, instead of viewing the decomposition operator $\widetilde{\mathcal{W}}_{J}$ and the reconstruction operator $\mathcal{V}_{J}$ in the recursive way as in (4.1) and (4.2), we consider $w_{j ; \ell}$ as a direct consequence of a linear mapping acting on the input signal $v$ (more precisely, $v_{J}$ ).

We have the following result on the recursive application of subdivision or transition operators.

Lemma 4.3. Let $\mathrm{M}_{1}, \mathrm{M}_{2}$ be $d \times d$ invertible integer matrices and let $u_{1}, u_{2} \in l_{0}\left(\mathbb{Z}^{d}\right)$. Then

$$
\mathcal{S}_{u_{1}, \mathrm{M}_{1}} \mathcal{S}_{u_{2}, \mathrm{M}_{2}} v=\mathcal{S}_{u_{1} *\left(u_{2} \uparrow \mathrm{M}_{1}\right), \mathrm{M}_{1} \mathrm{M}_{2}} v=\left|\operatorname{det}\left(\mathrm{M}_{1} \mathrm{M}_{2}\right)\right| u_{1} *\left(u_{2} \uparrow \mathrm{M}_{1}\right) *\left(v \uparrow \mathrm{M}_{1} \mathrm{M}_{2}\right)
$$

and

$$
\mathcal{T}_{u_{2}, \mathrm{M}_{2}} \mathcal{T}_{u_{1}, \mathrm{M}_{1}} v=\mathcal{T}_{u_{1} *\left(u_{2} \uparrow \mathrm{M}_{1}\right), \mathrm{M}_{1} \mathrm{M}_{2}} v=\left|\operatorname{det}\left(\mathrm{M}_{1} \mathrm{M}_{2}\right)\right|\left(u_{1} *\left(u_{2} \uparrow \mathrm{M}_{1}\right) * v\right) \downarrow \mathrm{M}_{1} \mathrm{M}_{2}
$$


Proof. The Fourier series of the sequence $\mathcal{S}_{u_{1}, \mathrm{M}_{1}} \mathcal{S}_{u_{2}, \mathrm{M}_{2}} v$ is

$$
\left|\operatorname{det}\left(\mathrm{M}_{1}\right)\right| \widehat{u_{1}}(\xi) \widehat{\mathcal{S}_{u_{2}, \mathrm{M}_{2}}} v\left(\mathrm{M}_{1}^{\top} \xi\right)=\left|\operatorname{det}\left(\mathrm{M}_{1} \mathrm{M}_{2}\right)\right| \widehat{u_{1}}(\xi) \widehat{u_{2}}\left(\mathrm{M}_{1}^{\top} \xi\right) \widehat{v}\left(\mathrm{M}_{2}^{\top} \mathrm{M}_{1}^{\top} \xi\right)
$$

Therefore, (4.20) holds. By duality in (2.18) and by (4.20), we have

$$
\begin{aligned}
\left\langle w, \mathcal{T}_{u_{2}, \mathrm{M}_{2}} \mathcal{T}_{u_{1}, \mathrm{M}_{1}} v\right\rangle & =\left\langle\mathcal{S}_{u_{2}, \mathrm{M}_{2}} w, \mathcal{T}_{u_{1}, \mathrm{M}_{1}} v\right\rangle=\left\langle\mathcal{S}_{u_{1}, \mathrm{M}_{1}} \mathcal{S}_{u_{2}, \mathrm{M}_{2}} w, v\right\rangle \\
& =\left\langle\mathcal{S}_{u_{1} *\left(u_{2} \uparrow \mathrm{M}_{1}\right), \mathrm{M}_{1} \mathrm{M}_{2}} w, v\right\rangle=\left\langle w, \mathcal{T}_{u_{1} *\left(u_{2} \uparrow \mathrm{M}_{1}\right), \mathrm{M}_{1} \mathrm{M}_{2}} v\right\rangle,
\end{aligned}
$$

from which we see that (4.21) holds.

From the definition of the sequence $w_{j ; \ell}$ of framelet coefficients in (4.1), we see that

$$
v_{j}=\mathrm{d}_{\mathrm{M}}^{-1 / 2} \mathcal{T}_{\tilde{a}, \mathrm{M}} v_{j+1}=\cdots=\mathrm{d}_{\mathrm{M}}^{(j-J) / 2} \mathcal{T}_{\tilde{a}, \mathrm{M}}^{J-j} v_{J}=\mathrm{d}_{\mathrm{M}}^{(j-J) / 2} \mathcal{T}_{\tilde{a} *(\tilde{a} \uparrow \mathrm{M}) * \cdots *\left(\tilde{a} \uparrow \mathrm{M}^{J-j-1}\right), \mathrm{M}^{J-j}} v
$$

and

$$
w_{j ; \ell}=\mathrm{d}_{\mathrm{M}}^{-1 / 2} \mathcal{T}_{\tilde{b}_{\ell}} v_{j+1}=\mathrm{d}_{\mathrm{M}}^{(j-J) / 2} \mathcal{T}_{\tilde{b}_{\ell}, \mathrm{M}} \mathcal{T}_{\tilde{a}, \mathrm{M}}^{(j+1-J) / 2} v_{J}=\mathrm{d}_{\mathrm{M}}^{(j-J) / 2} \mathcal{T}_{\tilde{a} *(\tilde{a} \uparrow \mathrm{M}) * \cdots *\left(\tilde{a} \uparrow \mathrm{M}^{J-j-2}\right) *\left(\tilde{b}_{\ell} \uparrow \mathrm{M}^{J-j-1}\right), \mathrm{M}^{J-j}} v .
$$

Therefore, framelet coefficients $w_{j-1 ; \ell}$ are obtained by filtering a given signal $v$ using the filters $\mathrm{d}_{\mathrm{M}}^{(J-j) / 2} \tilde{a} *$ $(\tilde{a} \uparrow \mathrm{M}) * \cdots *\left(\tilde{a} \uparrow \mathrm{M}^{J-j-1}\right) *\left(\tilde{b}_{\ell} \uparrow \mathrm{M}^{J-j}\right)$, whose supports grow with the scale level $J-j$. More precisely, by (2.2),

$$
w_{j ; \ell}(\mathrm{k})=\left\langle v, \mathrm{~d}_{\mathrm{M}}^{(J-j) / 2}\left[\tilde{a} *(\tilde{a} \uparrow \mathrm{M}) * \cdots *\left(\tilde{a} \uparrow \mathrm{M}^{J-j-2}\right) *\left(\tilde{b}_{\ell} \uparrow \mathrm{M}^{J-j-1}\right)\right]\left(\cdot-\mathrm{M}^{J-j} \mathrm{k}\right)\right\rangle, \quad \mathrm{k} \in \mathbb{Z}^{d} .
$$

Similarly, we deduce that

$$
\mathcal{V}_{J}\left(0, \ldots, 0, v_{0}\right)=\mathrm{d}_{\mathrm{M}}^{-J / 2} \mathcal{S}_{a, \mathrm{M}}^{J} v_{0}=\mathrm{d}_{\mathrm{M}}^{(j-J) / 2} \mathcal{S}_{a *(a \uparrow \mathrm{M}) * \cdots *\left(a \uparrow \mathrm{M}^{J-1}\right), \mathrm{M}^{J}} v_{0}
$$

and

$$
\begin{aligned}
\mathcal{V}_{J}\left(0, \ldots, 0, w_{j ; \ell}, 0, \ldots, 0\right) & =\mathrm{d}_{\mathrm{M}}^{(j-J) / 2} \mathcal{S}_{a, \mathrm{M}}^{J-j-1} \mathcal{S}_{b_{\ell}, \mathrm{M}} w_{j ; \ell} \\
& =\mathrm{d}_{\mathrm{M}}^{(j-J) / 2} \mathcal{S}_{a *(\tilde{a} \uparrow \mathrm{M}) * \cdots *\left(a \uparrow \mathrm{M}^{J-j-2}\right) *\left(b_{\ell} \uparrow \mathrm{M}^{J-j-1}\right), \mathrm{M}^{J-j}} w_{j ; \ell} .
\end{aligned}
$$

Define filters $a_{j}$ and $\tilde{a}_{j}, j \in \mathbb{N}$ by

$$
\widehat{a_{j}}(\xi):=\widehat{a}(\xi) \widehat{a}\left(\mathrm{M}^{\top} \xi\right) \cdots \widehat{a}\left(\left(\mathrm{M}^{\top}\right)^{j-1} \xi\right) \quad \text { and } \quad \widehat{\widetilde{a}}(\xi):=\widehat{\tilde{a}}(\xi) \widehat{\tilde{a}}\left(\mathrm{M}^{\top} \xi\right) \cdots \widehat{\tilde{a}}\left(\left(\mathrm{M}^{\top}\right)^{j-1} \xi\right)
$$

with the convention that $a_{0}=\tilde{a}_{0}:=\delta$. That is,

$$
a_{j}=a *(a \uparrow \mathrm{M}) * \cdots *\left(a \uparrow \mathrm{M}^{j-1}\right) \quad \text { and } \quad \tilde{a}_{j}:=\tilde{a} *(\tilde{a} \uparrow \mathrm{M}) * \cdots *\left(\tilde{a} \uparrow \mathrm{M}^{j-1}\right) .
$$

Now a $J$-level discrete framelet transform employing a dual M-framelet filter bank $\left(\left\{\tilde{a} ; \tilde{b}_{1}, \ldots, \tilde{b}_{s}\right\}\right.$, $\left.\left\{a ; b_{1}, \ldots, b_{s}\right\}\right)$ can be equivalently rewritten as

$$
v=\sum_{\mathbf{k} \in \mathbb{Z}^{d}}\left\langle v, \tilde{a}_{[J ; \mathbf{k}]}\right\rangle a_{[J ; \mathbf{k}]}+\sum_{j=1}^{J} \sum_{\mathbf{k} \in \mathbb{Z}^{d}} \sum_{\ell=1}^{s}\left\langle v, \tilde{b}_{\ell,[j ; \mathbf{k}]}\right\rangle b_{\ell,[j ; \mathbf{k}]},
$$

where

$$
a_{[j ; \mathrm{k}]}:=\mathrm{d}_{\mathrm{M}}^{j / 2} a_{j}\left(\cdot-\mathrm{M}^{j} \mathrm{k}\right), \quad b_{\ell,[j ; \mathrm{k}]}:=\mathrm{d}_{\mathrm{M}}^{j / 2}\left[a_{j-1} *\left(b_{\ell} \uparrow \mathrm{M}^{j-1}\right)\right]\left(\cdot-\mathrm{M}^{j} \mathrm{k}\right)
$$

and

$$
\tilde{a}_{[j ; \mathrm{k}]}:=\mathrm{d}_{\mathrm{M}}^{j / 2} \tilde{a}_{j}\left(\cdot-\mathrm{M}^{j} \mathrm{k}\right), \quad \tilde{b}_{\ell,[j ; \mathrm{k}]}:=\mathrm{d}_{\mathrm{M}}^{j / 2}\left[\tilde{a}_{j-1} *\left(\tilde{b}_{\ell} \uparrow \mathrm{M}^{j-1}\right)\right]\left(\cdot-\mathrm{M}^{j} \mathrm{k}\right) .
$$


By employing the dilation matrix $\mathrm{M}$, a multilevel discrete framelet transform provides a multi-scale representation of a signal, which is the key to extract the multiscale structure in a signal. The representation in (4.27) also shows that the stability of a multilevel discrete framelet transform in the space $l_{2}\left(\mathbb{Z}^{d}\right)$ is closely related to the asymptotic behavior of the sequences $a_{J}, \tilde{a}_{J}$ in (4.25) as $J \rightarrow+\infty$, which is in turn closely related to the frequency-based refinable functions $\varphi$ and $\tilde{\varphi}$.

The above discussion motivates us to define discrete affine systems as follows:

$$
\begin{aligned}
\operatorname{DAS}_{J}\left(\left\{a ; b_{1}, \ldots, b_{s}\right\}\right):= & \left\{a_{[J ; \mathrm{k}]}: \mathrm{k} \in \mathbb{Z}^{d}\right\} \\
& \cup\left\{b_{\ell,[j ; \mathrm{k}]}: \ell=1, \ldots, s, j=1, \ldots, J, \mathrm{k} \in \mathbb{Z}^{d}\right\}
\end{aligned}
$$

and

$$
\begin{aligned}
\operatorname{DAS}_{J}\left(\left\{\tilde{a} ; \tilde{b}_{1}, \ldots, \tilde{b}_{s}\right\}\right):= & \left\{\tilde{a}_{[J ; \mathrm{k}]}: \mathrm{k} \in \mathbb{Z}^{d}\right\} \\
& \cup\left\{\tilde{b}_{\ell,[j ; \mathrm{k}]}: \ell=1, \ldots, s, j=1, \ldots, J, \mathrm{k} \in \mathbb{Z}^{d}\right\} .
\end{aligned}
$$

Under the convention that

$$
\sim: \operatorname{DAS}_{J}\left(\left\{a ; b_{1}, \ldots, b_{s}\right\}\right) \rightarrow \operatorname{DAS}_{J}\left(\left\{\tilde{a} ; \tilde{b}_{1}, \ldots, \tilde{b}_{s}\right\}\right) \quad \text { with } \quad u \mapsto \tilde{u},
$$

that is, $(u, \tilde{u})$ is always regarded as a pair together, the representation of $v \in l_{2}\left(\mathbb{Z}^{d}\right)$ in (4.27) can be rewritten as

$$
v=\sum_{u \in \operatorname{DAS}_{J}\left(\left\{a ; b_{1}, \ldots, b_{s}\right\}\right)}\langle v, \tilde{u}\rangle u, \quad v \in l_{2}\left(\mathbb{Z}^{d}\right), J \in \mathbb{N} .
$$

Therefore, the stability in (4.6) of a filter bank $\left\{a ; b_{1}, \ldots, b_{s}\right\}$ in $l_{2}\left(\mathbb{Z}^{d}\right)$ simply means the stability of the discrete affine system $\operatorname{DAS}_{J}\left(\left\{a ; b_{1}, \ldots, b_{s}\right\}\right)$ in $l_{2}\left(\mathbb{Z}^{d}\right)$ :

$$
C_{1}^{2}\|v\|_{l_{2}\left(\mathbb{Z}^{d}\right)}^{2} \leqslant \sum_{u \in \operatorname{DAS}_{J}\left(\left\{a ; b_{1}, \ldots, b_{s}\right\}\right)}|\langle v, u\rangle|^{2} \leqslant C_{2}^{2}\|v\|_{l_{2}\left(\mathbb{Z}^{d}\right)}^{2}, \quad \forall v \in l_{2}\left(\mathbb{Z}^{d}\right), J \in \mathbb{N} .
$$

In other words, if $\left\{a ; b_{1}, \ldots, b_{s}\right\}$ has stability in $l_{2}\left(\mathbb{Z}^{d}\right)$, then $\operatorname{DAS}_{J}\left(\left\{a ; b_{1}, \ldots, b_{s}\right\}\right)$ is a frame for $L_{2}\left(\mathbb{R}^{d}\right)$ for all $J \in \mathbb{N}$. It is also easy to prove that $\left\{a ; b_{1}, \ldots, b_{s}\right\}$ is a tight M-framelet filter bank if and only if (4.33) holds with $C_{1}=C_{2}=1$. Furthermore, $\left\{a ; b_{1}, \ldots, b_{\mathrm{d}_{\mathrm{M}}}\right\}$ is an orthogonal M-wavelet filter bank if and only if $\operatorname{DAS}_{J}\left(\left\{a ; b_{1}, \ldots, b_{\mathrm{d}_{\mathrm{M}}}\right\}\right)$ is an orthonormal basis for $l_{2}\left(\mathbb{Z}^{d}\right)$ for every $J \in \mathbb{N}$.

\subsection{Variants of multilevel discrete framelet transforms}

There are many variants to a standard multilevel discrete framelet transform. Here we look at two particular variants. By the definition in (2.1) and (2.2), it is easy to notice that

$$
\mathcal{S}_{u, \mathrm{M}}(v(\cdot-\mathrm{n}))=\left[\mathcal{S}_{u, \mathrm{M}} v\right](\cdot-\mathrm{Mn}), \quad \mathcal{S}_{u(\cdot-\mathrm{n}), \mathrm{M}} v=\left[\mathcal{S}_{u, \mathrm{M}} v\right](\cdot-\mathrm{n}), \quad \mathrm{n} \in \mathbb{Z}^{d}
$$

and

$$
\mathcal{T}_{u, \mathrm{M}}(v(\cdot-\mathrm{Mn}))=\left[\mathcal{T}_{u, \mathrm{M}} v\right](\cdot-\mathrm{n}), \quad \mathcal{T}_{u(\cdot+\mathrm{n}), \mathrm{M}} v=\mathcal{T}_{u, \mathrm{M}}(v(\cdot-\mathrm{n})), \quad \mathrm{n} \in \mathbb{Z}^{d} .
$$

In other words, if we shift an input signal $v$ or a filter $u$ by an integer, then its output under the subdivision operator is a shifted version of $\mathcal{S}_{u, \mathrm{M}} v$. For the transition operator, however, $\mathcal{T}_{u, \mathrm{M}}(v(\cdot-\mathrm{n}))$ or $\mathcal{T}_{u(\cdot+\mathrm{n}), \mathrm{M} v}$ is generally no longer a shifted version of $\mathcal{T}_{u, \mathrm{M}} v$ if $\mathrm{n} \notin \mathrm{M} \mathbb{Z}^{d}$. This shift sensitivity of framelet coefficients with respect to a shift of an input signal is not desirable in some applications such as signal or image denoising, since a simple shift of a noise wouldn't change the characteristics of a noise. To overcome this difficulty, we put together the $\mathrm{d}_{\mathrm{M}}$ sequences $\mathcal{T}_{u(\cdot+\gamma), \mathrm{M}} v, \gamma \in \Gamma_{\mathrm{M}}$ in a disjoint way so that we have only one sequence $\mathrm{d}_{\mathrm{M}} u^{\star} * v$. Similarly, it is easy to verify that $\sum_{\gamma \in \Gamma_{\mathrm{M}}} \mathcal{S}_{u(\cdot+\gamma), \mathrm{M}}(w(\mathrm{M} \cdot-\gamma))=\mathrm{d}_{\mathrm{M}} u * w$. In other words, the new discrete framelet transform is undecimated by removing the downsampling (i.e., decimation) and upsampling operations in the original discrete framelet transform. Thus, it is called $a$ 
$J$-level discrete undecimated framelet transform employing the filter bank $\left(\left\{\tilde{a} ; \tilde{b}_{1}, \ldots, \tilde{b}_{s}\right\},\left\{a ; b_{1}, \ldots, b_{s}\right\}\right)$, which in the terminology of Subsection 4.1 is just a $J$-level discrete framelet transform employing the new filter bank

$$
\begin{aligned}
& \left(\mathrm{d}_{\mathrm{M}}^{-1 / 2}\left\{\tilde{a}\left(\cdot-\gamma_{1}\right), \ldots, \tilde{a}\left(\cdot-\gamma_{\mathrm{d}_{\mathrm{M}}}\right) ; \tilde{b}_{1}\left(\cdot-\gamma_{1}\right), \ldots, \tilde{b}_{1}\left(\cdot-\gamma_{\mathrm{d}_{\mathrm{M}}}\right), \ldots, \tilde{b}_{s}\left(\cdot-\gamma_{1}\right), \ldots, \tilde{b}_{s}\left(\cdot-\gamma_{\mathrm{d}_{\mathrm{M}}}\right)\right\},\right. \\
& \left.\mathrm{d}_{\mathrm{M}}^{-1 / 2}\left\{a\left(\cdot-\gamma_{1}\right), \ldots, a\left(\cdot-\gamma_{\mathrm{d}_{\mathrm{M}}}\right) ; b_{1}\left(\cdot-\gamma_{1}\right), \ldots, b_{1}\left(\cdot-\gamma_{\mathrm{d}_{\mathrm{M}}}\right), \ldots, b_{s}\left(\cdot-\gamma_{1}\right), \ldots, b_{s}\left(\cdot-\gamma_{\mathrm{d}_{\mathrm{M}}}\right)\right\}\right),
\end{aligned}
$$

where $\left\{\gamma_{1}, \ldots, \gamma_{\mathrm{d}_{\mathrm{M}}}\right\}:=\Gamma_{\mathrm{M}}$. Let us present a $J$-level discrete undecimated framelet transform employing a dual M-framelet filter bank $\left(\left\{\tilde{a} ; \tilde{b}_{1}, \ldots, \tilde{b}_{s}\right\},\left\{a ; b_{1}, \ldots, b_{s}\right\}\right)$. A J-level discrete undecimated framelet decomposition is given by

$$
v_{j-1}:=\left(\tilde{a}^{\star} \uparrow \mathrm{M}^{J-j}\right) * v_{j}, \quad w_{j-1 ; \ell}:=\left(\tilde{b}_{\ell}^{\star} \uparrow \mathrm{M}^{J-j}\right) * v_{j}, \quad \ell=1, \ldots, s, j=J, \ldots, 1,
$$

where $v_{J}: \mathbb{Z}^{d} \rightarrow \mathbb{C}$ is an input signal. A J-level discrete undecimated framelet reconstruction is

$$
\stackrel{\circ}{v}_{j}:=\left(a \uparrow \mathrm{M}^{J-j}\right) * \stackrel{\circ}{v}_{j-1}+\sum_{\ell=1}^{s}\left(b_{\ell} \uparrow \mathrm{M}^{J-j}\right) * \stackrel{\circ}{w}_{j-1 ; \ell}, \quad j=1, \ldots, J .
$$

Using the same proof as in Theorem 2.1, one can straightforwardly check that the above $J$-level discrete undecimated framelet transform has the perfect reconstruction property if and only if

$$
\widehat{\tilde{a}}(\xi) \overline{\widehat{a}(\xi)}+\widehat{\tilde{b}_{1}}(\xi) \overline{\widehat{b}_{1}(\xi)}+\cdots+\widehat{\tilde{b}}_{s}(\xi) \overline{\widehat{b}_{s}(\xi)}=1 .
$$

Therefore, the perfect reconstruction condition on a filter bank for an undecimated framelet transform is much weaker than the condition in (4.5) for an usual dual M-framelet filter bank. In this sense, an undecimated framelet transform would be properly called a multiscale convolution transform using a filter bank satisfying (4.38). Similar to (4.27), we have the signal representation:

$$
v=\sum_{\mathbf{k} \in \mathbb{Z}^{d}}\left\langle v, \tilde{a}_{J}(\cdot-\mathbf{k})\right\rangle a_{J}(\cdot-\mathbf{k})+\sum_{j=1}^{J} \sum_{\mathbf{k} \in \mathbb{Z}^{d}} \sum_{\ell=1}^{s}\left\langle v, \tilde{b}_{\ell,[j ; \mathbf{0}]}(\cdot-\mathbf{k})\right\rangle b_{\ell,[j ; \mathbf{0}]}(\cdot-\mathbf{k}) .
$$

A $J$-level discrete undecimated framelet transform has redundancy ratio $J s$ while the original $J$-level discrete framelet transform has redundancy ratio $\frac{s\left(\mathrm{~d}_{M}^{J-1}-1\right)}{\mathrm{d}_{M}^{J-1}\left(\mathrm{~d}_{\mathrm{M}}-1\right)}+\frac{1}{\mathrm{~d}_{\mathrm{M}}^{J-1}}$ (a $J$-level discrete wavelet transform has redundancy ratio one, that is, no redundancy). Therefore, a $J$-level discrete undecimated framelet transform is a redundant transform, having roughly $\mathrm{d}_{\mathrm{M}} J$ times more coefficients than the original $J$-level discrete framelet transform, but has a simple structure. To reduce the redundancy rate and to keep the transform nearly shift invariant, we discuss another variant - a discrete averaging framelet transform.

Let $n$ be a positive integer. We say that $\mathcal{A}:=\left(\mathcal{A}_{1}, \ldots, \mathcal{A}_{n}\right): l\left(\mathbb{Z}^{d}\right) \rightarrow\left(l\left(\mathbb{Z}^{d}\right)\right)^{1 \times n}, \mathcal{A} v=\left(\mathcal{A}_{1} v, \ldots, \mathcal{A}_{n} v\right)$ is a data partitioning operator if $\mathcal{A}$ is an injective operator (that is, a one-to-one but not necessarily linear mapping). $\mathcal{A}$ has at least one left-inverse and we say that $\tilde{\mathcal{A}}:\left(l\left(\mathbb{Z}^{d}\right)\right)^{1 \times n} \rightarrow l\left(\mathbb{Z}^{d}\right)$ is a data averaging operator of $\mathcal{A}$ if $\tilde{\mathcal{A}} \mathcal{A} v=v$ for all $v \in l\left(\mathbb{Z}^{d}\right)$. An example of data partitioning and data averaging operators is to use the convolution operation. Let $\Theta_{1}, \ldots, \Theta_{n}, \tilde{\Theta}_{1}, \ldots, \tilde{\Theta}_{n} \in l_{0}(\mathbb{Z})$ be finitely supported sequences on $\mathbb{Z}^{d}$ such that

$$
\widehat{\tilde{\Theta}_{1}}(\xi) \overline{\widehat{\Theta}_{1}}(\xi)+\cdots+\widehat{\tilde{\Theta}_{n}}(\xi) \overline{\widehat{\Theta}_{n}(\xi)}=1 .
$$

Then we have a data partitioning operator $\mathcal{A}: l\left(\mathbb{Z}^{d}\right) \rightarrow\left(l\left(\mathbb{Z}^{d}\right)\right)^{1 \times n}, \mathcal{A} v=\left(\Theta_{1}^{\star} * v, \ldots, \Theta_{n}^{\star} * v\right)$ and a data averaging operator $\tilde{\mathcal{A}}:\left(l\left(\mathbb{Z}^{d}\right)\right)^{1 \times n} \rightarrow l\left(\mathbb{Z}^{d}\right), \tilde{\mathcal{A}}\left(v_{1}, \ldots, v_{n}\right)=\tilde{\Theta}_{1} * v_{1}+\cdots+\tilde{\Theta}_{n} * v_{n}$. By $(4.40)$, it is evident that $\tilde{\mathcal{A}} \mathcal{A}=\operatorname{Id}_{l\left(\mathbb{Z}^{d}\right)}$. A simple choice is to take $\Theta_{1}=\cdots=\Theta_{n}=\delta$ and $\tilde{\Theta}_{1}=\cdots=\tilde{\Theta}_{n}=\frac{1}{n} \delta$. For this particular example, the data partitioning operator simply copies the data $n$ times and the data averaging operator $\tilde{\mathcal{A}}$ is exactly the averaging operation.

Under a data partitioning operator $\mathcal{A}$, a signal $v$ is split into $n$ sub-signals: $\mathcal{A}_{1} v, \ldots, \mathcal{A}_{n} v$. Regard each sub-signal as a completely separate branch. Now a discrete $n$-branch averaging framelet transform is to 
perform an independent discrete framelet transform on each branch. After processing and reconstruction of each branch, we end up with $n$ reconstructed sub-signals. Then we use the data averaging operator to form one reconstructed signal.

A particular discrete framelet transform may perform better only for a small subset of signals with certain characteristics. Using a data partitioning operator, we may be able to split a signal into subsignals with different characteristics so that the advantages of a particular discrete framelet transform can be explored. Comparing with a $J$-level discrete undecimated framelet transform which has redundancy ratio $J s$, a $J$-level discrete $n$-branch averaging framelet transform has only $n$ times redundancy, which is independent of the scale level $J$ and is much smaller than $J s$ when the scale level $J$ is large. A discrete averaging framelet transform using complex-valued dual framelet filter banks is of particular interest in high-dimensional data analysis for achieving directional representations to capture various high-dimensional singularities such as edges in images. For example, the dual-tree complex wavelet transform employing two correlated orthogonal wavelet filter banks (see the tutorial article [38]) is a particular example of the averaging framelet transform with $n=2$ branches. See Section 7 for another example of directional framelets using tensor product complex-valued tight framelet filter banks.

\section{Linear-phase Moments and Symmetry Property of Framelets}

In this section we discuss linear-phase moments and symmetry property in wavelet analysis.

It is sometimes desirable that the image of a polynomial under a convolution operation is itself or its translated version. For this purpose, we recall the notion of linear-phase moments introduced in [23, 26]. We say that $u \in l_{0}\left(\mathbb{Z}^{d}\right)$ has order $m$ linear-phase moments with phase $\mathrm{c} \in \mathbb{R}^{d}$ if

$$
\widehat{u}(\xi)=e^{-i c \cdot \xi}+\mathcal{O}\left(\|\xi\|^{m}\right), \quad \xi \rightarrow \mathbf{0} .
$$

If $m>1$, it follows from (5.1) that $\mathrm{c}=\sum_{\mathrm{k} \in \mathbb{Z}^{d}} u(\mathrm{k}) \mathrm{k}$, which is called the default phase of $u$. If $u$ has order $m$ but not $m+1$ linear-phase moments with the default phase, then we define $\operatorname{lpm}(u):=m$ and we say that $u$ has the linear-phase moments of order $m$.

If a filter has linear-phase moments, then the action of the convolution operation, the subdivision operator, and the transition operator on polynomial spaces has some particular structure.

Proposition 5.1. Let $u \in l_{0}\left(\mathbb{Z}^{d}\right), \mathrm{c} \in \mathbb{R}^{d}$, and $m \in \mathbb{N}_{0}$. Then (i) $\mathrm{p} * u=\mathrm{p}\left(\cdot-\mathrm{c}\right.$ ) for all $\mathrm{p} \in \Pi_{m-1}$ if and only if (ii) $u$ has order $m$ linear-phase moments with phase $\mathrm{c}$ if and only if (iii) $\mathcal{T}_{u, \mathrm{MP}}=\mathrm{d}_{\mathrm{M}} \mathrm{P}(\mathrm{M} \cdot+\mathrm{c})$ for all $\mathrm{p} \in \Pi_{m-1}$. Similarly, $u$ has order $m$ sum rules and order $m$ linear-phase moments with phase $\mathrm{c}$ if and only if $\mathcal{S}_{u, \mathrm{MP}}=\mathrm{p}\left(\mathrm{M}^{-1}(\cdot-\mathrm{c})\right)$ for all $\mathrm{p} \in \Pi_{m-1}$.

Proof. By Lemma 3.1, we have (3.7). On the other hand, using the Taylor expansion of $\mathrm{p}$, we have $\mathrm{p}(x-\mathrm{c})=\sum_{\mu \in \mathbb{N}_{0}^{d},|\mu|<m} \partial^{\mu} \mathrm{p}(x) \frac{(-\mathrm{c})^{\mu}}{\mu !}$. Comparing the coefficients of $\partial^{\mu} \mathrm{p}, \mu \in \mathbb{N}_{0}^{d},|\mu|<m$ with (3.7), we see that $\mathrm{p} * u=\mathrm{p}(\cdot-\mathrm{c})$ if and only if $\partial^{\mu} \widehat{u}(0)=(-i \mathrm{c})^{\mu}$ for all $\mu \in \mathbb{N}_{0}^{d},|\mu|<m$, which can be equivalently rewritten as (5.1). (iii) is a direct consequence of item (i) and (3.10) in Theorem 3.2. The part on subdivision operator is a direct consequence of Theorem 3.5.

Linear-phase moments are of particular interest in the application of subdivision schemes in computer aided geometric design, since the property $\mathcal{S}_{u, \mathrm{M}} \mathrm{p}=\mathrm{p}\left(\mathrm{M}^{-1}(\cdot-\mathrm{c})\right)$ for all $\mathrm{p} \in \Pi_{m-1}$ simply means that the subdivision scheme is interpolating on all polynomial sequences $\mathrm{p} \in \Pi_{m-1}$.

In the following, we make some remarks on the relations between linear-phase moments and symmetry. Note that a filter $u$ has one linear-phase moment just means $\widehat{u}(0)=1$.

Proposition 5.2. Let $u \in l_{0}\left(\mathbb{Z}^{d}\right)$ satisfying $\operatorname{lpm}(u)>1$ with phase $c \in \mathbb{R}^{d}$.

(i) If $u\left(\mathrm{c}_{u}-\mathrm{k}\right)=u(\mathrm{k})$ for all $\mathrm{k} \in \mathbb{Z}^{d}$ with $\mathrm{c}_{u} \in \mathbb{Z}^{d}$, then $\mathrm{c}=\mathrm{c}_{u} / 2$ (that is, the phase $\mathrm{c}$ agrees with the symmetry center $\mathrm{c}_{u} / 2$ of $u$ ) and $\operatorname{lpm}(u)$ must be an even integer;

(ii) If $u\left(\mathrm{c}_{u}-\mathrm{k}\right)=\overline{u(\mathrm{k})}$ for all $\mathrm{k} \in \mathbb{Z}^{d}$ with $\mathrm{c}_{u} \in \mathbb{Z}^{d}$, then $\mathrm{c}=\mathrm{c}_{u} / 2$. 
Proof. Set $m:=\operatorname{lpm}(u)$. For (i), we have $\widehat{u}(\xi)=e^{-i c_{u} \cdot \xi} \widehat{u}(-\xi)$. Then it follows from (5.1) that $e^{-i c_{u} \cdot \xi}=$ $\frac{\widehat{u}(\xi)}{\widehat{u}(-\xi)}=e^{-i 2 \mathrm{c} \cdot \xi}+\mathcal{O}\left(\|\xi\|^{m}\right)$ as $\xi \rightarrow \mathbf{0}$. Since $m>1$, we must have $\mathbf{c}=\mathrm{c}_{u} / 2$. Note that $\widehat{u}(\xi)=e^{-i c_{u} \cdot \xi} \widehat{u}(-\xi)$ and $\mathrm{c}_{u}=2 \mathrm{c}$ imply $\widehat{u}(\xi) e^{i c \cdot \xi}=\widehat{u}(-\xi) e^{-i c \cdot \xi}$, from which we see that

$$
\partial^{\mu}\left[\widehat{u}(\xi) e^{i c \cdot \xi}\right](0)=0, \quad \text { for all } \mu \in \mathbb{N}_{0}^{d} \text { with positive odd integers }|\mu| .
$$

On the other hand, the definition of linear-phase moments in (5.1) is equivalent to $\widehat{u}(\xi) e^{i c \cdot \xi}=1+\mathcal{O}\left(\|\xi\|^{m}\right)$ as $\xi \rightarrow \mathbf{0}$. Since $u$ has $m$ but not $m+1$ linear-phase moments with phase c, it now follows from (5.2) that $m$ must be an even integer.

For (ii), we have $\widehat{u}(\xi)=e^{-i c_{u} \cdot \xi} \widehat{\widehat{u}(\xi)}$. Then it follows from (5.1) that we must have $e^{-i c_{u} \cdot \xi}=\frac{\widehat{u}(\xi)}{\widehat{\widehat{u}}(\xi)}=$ $e^{-i 2 c \cdot \xi}+\mathcal{O}\left(\|\xi\|^{m}\right)$ as $\xi \rightarrow \mathbf{0}$. Thus, $\mathrm{c}=\mathrm{c}_{u} / 2$ holds.

According to the following result, linear-phase moments also play a critical role in the construction of tight framelet filter banks having symmetry.

Proposition 5.3. Let $\left\{a ; b_{1}, \ldots, b_{s}\right\}$ be a tight $\mathrm{M}$-framelet filter bank such that $\widehat{a}(\mathbf{0})=1$ and $a\left(\mathrm{c}_{a}-\mathbf{k}\right)=$ $\overline{a(\mathrm{k})}$ for all $\mathrm{k} \in \mathbb{Z}^{d}$ with $\mathrm{c}_{a} \in \mathbb{Z}^{d}$. Then $\operatorname{lpm}\left(a^{\star} * a\right)=\operatorname{lpm}(a)$ and

$$
\min \left(\operatorname{vm}\left(b_{1}\right), \ldots, \operatorname{vm}\left(b_{s}\right)\right)=\min \left(\operatorname{sr}(a), \frac{1}{2} \operatorname{lpm}(a)\right) .
$$

Proof. By $\widehat{a}(\mathbf{0})=1$ we see from the perfect reconstruction condition (4.5) (also see [4,10]) that

$$
\min \left(\operatorname{vm}\left(b_{1}\right), \ldots, \operatorname{vm}\left(b_{s}\right)\right)=\min \left(\operatorname{sr}(a), \frac{1}{2} \operatorname{vm}\left(1-|\widehat{a}|^{2}\right)\right) .
$$

Since $a$ has symmetry, we have $\widehat{a}(\xi)=e^{-i c_{a} \cdot \xi \bar{a}(\xi)}$. Set $c:=\mathrm{c}_{a} / 2$ and $n:=\operatorname{vm}\left(1-|\widehat{a}|^{2}\right)$ Then,

$$
\left[1+e^{i c \cdot \xi} \widehat{a}(\xi)\right]\left[1-e^{i c \cdot \xi} \widehat{a}(\xi)\right]=1-|\widehat{a}(\xi)|^{2}=\mathcal{O}\left(\|\xi\|^{n}\right), \quad \xi \rightarrow \mathbf{0} .
$$

Since $\widehat{a}(\mathbf{0})=1$, we conclude from the above relation that $1-e^{i c \cdot \xi} \widehat{a}(\xi)=\mathcal{O}\left(\|\xi\|^{n}\right)$ as $\xi \rightarrow \mathbf{0}$. That is, we proved $\operatorname{lpm}(a) \geqslant n$. It is trivial that $\operatorname{vm}\left(1-|\widehat{a}|^{2}\right) \geqslant \operatorname{lpm}(a)$. Note that $\operatorname{vm}\left(1-|\widehat{a}|^{2}\right)=\operatorname{lpm}\left(a^{\star} * a\right)$. Hence, $\operatorname{lpm}\left(a^{\star} * a\right)=\operatorname{lpm}(a)$ and (5.3) follows directly from (5.4).

We now discuss symmetry properties of wavelets and framelets which are desirable in many applications. Let $\mathscr{G}$ be a finite subset of $d \times d$ integer matrices whose determinants are \pm 1 . We say that $\mathscr{G}$ is a symmetry group compatible with a $d \times d$ invertible integer matrix M (see $[17,18,21,22])$ if $\mathscr{G}$ forms a group under matrix multiplication and $\mathrm{M}^{-1} E \mathrm{M} \in \mathscr{G}$ for all $E \in \mathscr{G}$. That is, the mapping

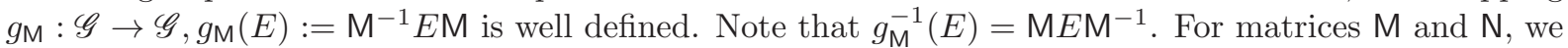
say that $\mathrm{N}$ is $\mathscr{G}$-equivalent to $\mathrm{M}$ if $\mathrm{N}=E \mathrm{M} F$ for some $E, F \in \mathscr{G}$. Note that $\left\{I_{d},-I_{d}\right\}$ is a basic symmetry group compatible with any $d \times d$ invertible integer matrix M. In dimension two, there are two important symmetry groups:

$$
\begin{aligned}
& D_{4}:=\left\{ \pm\left[\begin{array}{ll}
1 & 0 \\
0 & 1
\end{array}\right], \pm\left[\begin{array}{cc}
1 & 0 \\
0 & -1
\end{array}\right], \pm\left[\begin{array}{ll}
0 & 1 \\
1 & 0
\end{array}\right], \pm\left[\begin{array}{cc}
0 & 1 \\
-1 & 0
\end{array}\right]\right\}, \\
& D_{6}:=\left\{ \pm\left[\begin{array}{ll}
1 & 0 \\
0 & 1
\end{array}\right], \pm\left[\begin{array}{ll}
0 & -1 \\
1 & -1
\end{array}\right], \pm\left[\begin{array}{ll}
-1 & 1 \\
-1 & 0
\end{array}\right], \pm\left[\begin{array}{ll}
0 & 1 \\
1 & 0
\end{array}\right], \pm\left[\begin{array}{ll}
1 & -1 \\
0 & -1
\end{array}\right], \pm\left[\begin{array}{ll}
-1 & 0 \\
-1 & 1
\end{array}\right]\right\} .
\end{aligned}
$$

Define

$$
\mathrm{M}_{\sqrt{2}}:=\left[\begin{array}{cc}
1 & 1 \\
1 & -1
\end{array}\right], \quad \mathrm{M}_{\sqrt{3}}:=\left[\begin{array}{ll}
1 & -2 \\
2 & -1
\end{array}\right], \quad I_{2}:=\left[\begin{array}{ll}
1 & 0 \\
0 & 1
\end{array}\right] .
$$

As shown in [21, Theorem 2], up to $D_{4}$-equivalence and a multiplicative constant, $\mathrm{M}_{\sqrt{2}}$ and $I_{2}$ are the only two matrices which are compatible with $D_{4}$. Similarly, up to $D_{6}$-equivalence and a multiplicative constant, $\mathrm{M}_{\sqrt{3}}$ and $I_{2}$ are the only two matrices which are compatible with $D_{6}$. 
Let $a \in l_{0}\left(\mathbb{Z}^{d}\right)$ and $\mathscr{G}$ be a symmetry group on $\mathbb{Z}^{d}$. We say that $a$ has $\mathscr{G}$-symmetry with a symmetry center $\mathrm{c}_{a} \in \mathbb{R}^{d}$ and $\epsilon_{a} \in\{-1,1\}$ if

$$
a\left(E\left(\mathrm{k}-\mathrm{c}_{a}\right)+\mathrm{c}_{a}\right)=\epsilon_{a} a(\mathrm{k}), \quad \forall \mathrm{k} \in \mathbb{Z}^{d}, E \in \mathscr{G} .
$$

Clearly, we must have $\left(I_{d}-E\right) \mathrm{c}_{a} \in \mathbb{Z}^{d}$ for all $E \in \mathscr{G}$. Similarly, we say that a has complex $\mathscr{G}$-symmetry with a symmetry center $\mathrm{c}_{a} \in \mathbb{R}^{d}$ and $\epsilon_{a} \in\{-1,1\}$ if

$$
a\left(E\left(\mathrm{k}-\mathrm{c}_{a}\right)+\mathrm{c}_{a}\right)=\epsilon_{a} \overline{a(\mathrm{k})}, \quad \forall \mathrm{k} \in \mathbb{Z}^{d}, E \in \mathscr{G} .
$$

Now we have the following result on symmetry of an M-refinable function and its mask.

Theorem 5.4. ([22, Proposition 2.1]) Let $\mathrm{M}$ be a $d \times d$ expansive integer matrix. Let $\mathscr{G}$ be a symmetry group compatible with $\mathrm{M}$. Let $a \in l_{0}\left(\mathbb{Z}^{d}\right)$ with $\widehat{a}(\mathbf{0})=1$. Define $\boldsymbol{\varphi}$ as in (4.16) and a compactly supported distribution $\phi$ by $\widehat{\phi}=\boldsymbol{\varphi}$, that is, $\phi(x)=\frac{1}{(2 \pi)^{d}} \int_{\mathbb{R}^{d}} \varphi(\xi) e^{i x \cdot \xi} d \xi$ if $\varphi \in L_{1}\left(\mathbb{R}^{d}\right)$. Then a has $\mathscr{G}_{\text {-symmetry }}$ with a symmetry center $\mathrm{c}_{a}$ and $\epsilon_{a}=1$ if and only if

$$
\phi\left(E\left(\cdot-\mathrm{c}_{\phi}\right)+\mathrm{c}_{\phi}\right)=\phi, \quad \forall E \in \mathscr{G} \quad \text { with } \quad \mathrm{c}_{\phi}:=\left(\mathrm{M}-I_{d}\right)^{-1} \mathrm{c}_{a} .
$$

Moreover, if $\mathrm{N}=E \mathrm{M} F$ for some $E, F \in \mathscr{G}$ (that is, $\mathrm{N}$ is $\mathscr{G}$-equivalent to $\mathrm{M}$ ), then $\mathrm{N}$ is also expansive, $\phi^{\mathrm{N}}=\phi\left(\cdot+\left(\mathrm{M}-I_{d}\right)^{-1} \mathrm{c}_{a}-\left(\mathrm{N}-I_{d}\right)^{-1} \mathrm{c}_{a}\right)$, and

$$
\phi^{\mathrm{N}}\left(E\left(\cdot-\mathrm{c}_{\mathrm{N}}\right)+\mathrm{c}_{\mathrm{N}}\right)=\phi^{\mathrm{N}}, \quad \forall E \in \mathscr{G} .
$$

where $\mathrm{c}_{\mathrm{N}}:=\left(\mathrm{N}-I_{d}\right)^{-1} \mathrm{c}_{a}$ and $\widehat{\phi^{\mathrm{N}}}(\xi):=\prod_{j=1}^{\infty} \widehat{a}\left(\left(\mathrm{~N}^{\top}\right)^{-j} \xi\right), \xi \in \mathbb{R}^{d}$. If $b \in l_{0}\left(\mathbb{Z}^{d}\right)$ and $\mathscr{G}_{b}$ is a subgroup of $\mathscr{G}$ such that $b$ has $\mathscr{G}_{b}$-symmetry with a symmetry center $\mathrm{c}_{b}$ and $\epsilon_{b} \in\{-1,1\}$, then

$$
\psi\left(F\left(\cdot-\mathrm{c}_{\psi}\right)+\mathrm{c}_{\psi}\right)=\epsilon_{b} \psi, \quad \forall F \in \mathscr{G}_{b} \quad \text { with } \quad \mathrm{c}_{\psi}:=\mathrm{M}^{-1} \mathrm{c}_{b}+\mathrm{M}^{-1}\left(\mathrm{M}-I_{d}\right)^{-1} \mathrm{c}_{a},
$$

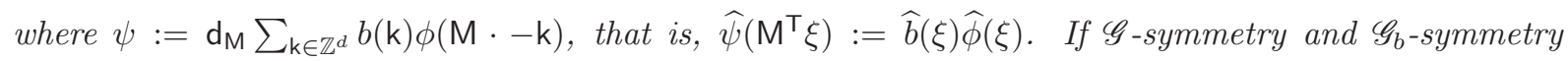

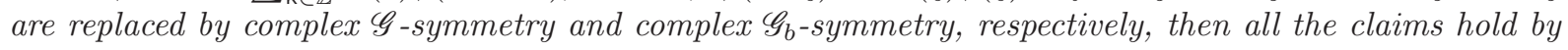
adding complex conjugate to the right sides of (5.10), (5.11), and (5.12).

Proof. Note that (5.10) is equivalent to

$$
\widehat{\phi}\left(E^{\top} \xi\right)=e^{i\left(\mathrm{c}_{a}-E \mathrm{c}_{a}\right) \cdot \xi} \widehat{\phi}(\xi)=e^{i\left(I_{d}-E\right)\left(\mathrm{M}-I_{d}\right)^{-1} \mathrm{c}_{a} \cdot \xi} \widehat{\phi}(\xi), \quad \forall E \in \mathscr{G}, \xi \in \mathbb{R}^{d} .
$$

Also note that (5.8) holds if and only if

$$
\widehat{a}\left(E^{\top} \xi\right)=\epsilon_{a} e^{i\left(I_{d}-E\right) c_{a} \cdot \xi} \widehat{a}(\xi), \quad E \in \mathscr{G}, \xi \in \mathbb{R}^{d} .
$$

By the definition of $\widehat{\phi}$ in (4.16) and the relation $\widehat{\phi}\left(\mathrm{M}^{\top} \xi\right)=\widehat{a}(\xi) \widehat{\phi}(\xi)$, one can easily check that (5.14) holds if and only if (5.13) holds.

Since $\mathscr{G}$ is finite and $\lim _{j \rightarrow \infty} \mathrm{M}^{-j}=0$, we must have $\lim _{j \rightarrow \infty} \mathrm{N}^{-j}=0$. By the definition of $\widehat{\phi}$ and $\widehat{\phi^{N}}$, we can directly check that $\widehat{\phi^{\mathrm{N}}}(\xi)=e^{i\left[\left(\mathrm{M}-I_{d}\right)^{-1}-\left(\mathrm{N}-I_{d}\right)^{-1}\right] \mathrm{c}_{a} \cdot \xi} \widehat{\phi}(\xi)$ is satisfied and (5.11) holds. Using (5.13) and $\widehat{b}\left(F^{\top} \xi\right)=\epsilon_{b} e^{i\left(I_{d}-F\right) c_{a} \cdot \xi} \widehat{b}(\xi)$ for all $F \in \mathscr{G}_{b}$, we have (5.12).

\section{Connections of Filter Banks to Frequency-based Dual Framelets}

In this section we discuss the connections between dual framelet filter banks and frequency-based dual framelets. We shall see that every dual framelet filter bank $\left(\left\{\tilde{a} ; \tilde{b}_{1}, \ldots, \tilde{b}_{s}\right\},\left\{a ; b_{1}, \ldots, b_{s}\right\}\right)$ satisfying $\widehat{\tilde{a}}(0)=\widehat{a}(0)=1$ corresponds to a frequency-based dual framelet on $\mathbb{R}^{d}$, which is introduced in $[27,28]$. For simplicity of presentation, we only outline the main ideas of the proofs and refer the reader to [27, 28] for the detailed proofs of the results stated in this section. At the end of this section, we mention some 
connections between the sparsity of a discrete framelet transform and the sparse representation of a dual framelet in the function space $L_{2}\left(\mathbb{R}^{d}\right)$.

Following the standard notation, we denote by $\mathscr{D}\left(\mathbb{R}^{d}\right)$ the linear space of all compactly supported $C^{\infty}$ functions. By $L_{p}^{\text {loc }}\left(\mathbb{R}^{d}\right)$ we denote the linear space of all measurable functions $f$ such that $\int_{\left\{y \in \mathbb{R}^{d}:\|y\| \leqslant r\right\}}|f(x)|^{p} d x<\infty$ for all $r>0$ with the usual modification for $p=\infty$. For $\mathbf{f} \in \mathscr{D}\left(\mathbb{R}^{d}\right)$ and $\psi \in L_{1}^{\text {loc }}\left(\mathbb{R}^{d}\right)$, we shall use the following pairing

$$
\langle\mathbf{f}, \boldsymbol{\psi}\rangle:=\int_{\mathbb{R}^{d}} \mathbf{f}(\xi) \overline{\boldsymbol{\psi}(\xi)} d \xi \quad \text { and } \quad\langle\boldsymbol{\psi}, \mathbf{f}\rangle:=\overline{\langle\mathbf{f}, \boldsymbol{\psi}\rangle}=\int_{\mathbb{R}^{d}} \boldsymbol{\psi}(\xi) \overline{\mathbf{f}(\xi)} d \xi .
$$

Let $J$ be an integer and $\mathrm{N}$ be a $d \times d$ invertible real-valued matrix. Let $\boldsymbol{\Phi}$ and $\boldsymbol{\Psi}$ be subsets of $L_{1}^{\text {loc }}\left(\mathbb{R}^{d}\right)$. A frequency-based $\left(\mathrm{N}^{\top}\right)^{-1}$-affine system (or an $\mathrm{N}$-modulation system) is defined to be

$$
\mathrm{FAS}_{J}(\boldsymbol{\Phi} ; \boldsymbol{\Psi})=\left\{\boldsymbol{\varphi}_{\mathrm{N}^{J} ; \mathbf{0}, \mathrm{k}}: \mathrm{k} \in \mathbb{Z}^{d}, \boldsymbol{\varphi} \in \boldsymbol{\Phi}\right\} \cup \cup_{j=J}^{\infty}\left\{\boldsymbol{\psi}_{\mathrm{N}^{j} ; \mathbf{0}, \mathrm{k}}: \mathrm{k} \in \mathbb{Z}^{d}, \boldsymbol{\psi} \in \boldsymbol{\Psi}\right\},
$$

where we used the notation in (1.1). To emphasize the role played by $\mathrm{N}$, we also use $\operatorname{FAS}_{J}^{\mathrm{N}}(\boldsymbol{\Phi} ; \boldsymbol{\Psi})$ instead of $\operatorname{FAS}_{J}(\boldsymbol{\Phi} ; \boldsymbol{\Psi})$. Let

$$
\boldsymbol{\Phi}=\left\{\boldsymbol{\varphi}^{1}, \ldots, \boldsymbol{\varphi}^{r}\right\}, \quad \tilde{\boldsymbol{\Phi}}=\left\{\tilde{\boldsymbol{\varphi}}^{1}, \ldots, \tilde{\boldsymbol{\varphi}}^{r}\right\}, \quad \boldsymbol{\Psi}=\left\{\boldsymbol{\psi}^{1}, \ldots, \boldsymbol{\psi}^{s}\right\}, \quad \tilde{\boldsymbol{\Psi}}=\left\{\tilde{\boldsymbol{\psi}}^{1}, \ldots, \tilde{\boldsymbol{\psi}}^{s}\right\}
$$

be subsets of $L_{1}^{\text {loc }}\left(\mathbb{R}^{d}\right)$. Let $\mathrm{FAS}_{J}(\boldsymbol{\Phi} ; \boldsymbol{\Psi})$ be defined in $(6.1)$ and $\mathrm{FAS}_{J}(\tilde{\boldsymbol{\Phi}} ; \tilde{\boldsymbol{\Psi}})$ be defined similarly. As in $[27,28]$, we say that the pair $\left(\operatorname{FAS}_{J}(\boldsymbol{\Phi} ; \boldsymbol{\Psi}), \operatorname{FAS}_{J}(\tilde{\boldsymbol{\Phi}} ; \tilde{\boldsymbol{\Psi}})\right)$ is a frequency-based dual $\left(\mathrm{N}^{\top}\right)^{-1}$-framelet if the following identity holds

$$
\sum_{\ell=1}^{r} \sum_{\mathbf{k} \in \mathbb{Z}^{d}}\left\langle\mathbf{f}, \boldsymbol{\varphi}_{\mathrm{N}^{j} ; \mathbf{0}, \mathrm{k}}^{\ell}\right\rangle\left\langle\tilde{\boldsymbol{\varphi}}_{\mathrm{N}^{j} ; \mathbf{0}, \mathrm{k}}^{\ell}, \mathbf{g}\right\rangle+\sum_{j=J}^{\infty} \sum_{\ell=1}^{s} \sum_{\mathbf{k} \in \mathbb{Z}^{d}}\left\langle\mathbf{f}, \boldsymbol{\psi}_{\mathrm{N}^{j} ; \mathbf{0}, \mathrm{k}}^{\ell}\right\rangle\left\langle\tilde{\boldsymbol{\psi}}_{\mathrm{N}^{j} ; \mathbf{0}, \mathrm{k}}^{\ell}, \mathbf{g}\right\rangle=(2 \pi)^{d}\langle\mathbf{f}, \mathbf{g}\rangle
$$

for all $\mathbf{f}, \mathbf{g} \in \mathscr{D}\left(\mathbb{R}^{d}\right)$, where the infinite series in (6.3) converge in the following sense

(i) for every $\mathbf{f}, \mathbf{g} \in \mathscr{D}\left(\mathbb{R}^{d}\right)$, all the following series converge absolutely for all integers $j \geqslant J$ :

$$
\sum_{\ell=1}^{r} \sum_{\mathbf{k} \in \mathbb{Z}^{d}}\left\langle\mathbf{f}, \boldsymbol{\varphi}_{\mathrm{N}^{j} ; \mathbf{0}, \mathrm{k}}^{\ell}\right\rangle\left\langle\tilde{\boldsymbol{\varphi}}_{\mathrm{N}^{J} ; \mathbf{0}, \mathrm{k}}^{\ell}, \mathbf{g}\right\rangle \quad \text { and } \sum_{\ell=1}^{s} \sum_{\mathrm{k} \in \mathbb{Z}^{d}}\left\langle\mathbf{f}, \boldsymbol{\psi}_{\mathrm{N}^{j} ; \mathbf{0}, \mathrm{k}}^{\ell}\right\rangle\left\langle\tilde{\boldsymbol{\psi}}_{\mathrm{N}^{j} ; \mathbf{0}, \mathrm{k}}^{\ell}, \mathbf{g}\right\rangle .
$$

(ii) for every $\mathbf{f}, \mathbf{g} \in \mathscr{D}\left(\mathbb{R}^{d}\right)$, the following limit exists and

$$
\begin{aligned}
\lim _{n \rightarrow+\infty}\left(\sum_{\ell=1}^{r} \sum_{\mathbf{k} \in \mathbb{Z}^{d}}\left\langle\mathbf{f}, \boldsymbol{\varphi}_{\mathrm{N}^{j} ; \mathbf{0}, \mathrm{k}}^{\ell}\right\rangle\left\langle\tilde{\boldsymbol{\varphi}}_{\mathrm{N}^{j} ; \mathbf{0}, \mathrm{k}}^{\ell}, \mathbf{g}\right\rangle\right. \\
\left.\quad+\sum_{j=J}^{n-1} \sum_{\ell=1}^{s} \sum_{\mathrm{k} \in \mathbb{Z}^{d}}\left\langle\mathbf{f}, \boldsymbol{\psi}_{\mathrm{N}^{j} ; \mathbf{0}, \mathrm{k}}^{\ell}\right\rangle\left\langle\tilde{\boldsymbol{\psi}}_{\mathrm{N}^{j} ; \mathbf{0}, \mathrm{k}}^{\ell}, \mathbf{g}\right\rangle\right)=(2 \pi)^{d}\langle\mathbf{f}, \mathbf{g}\rangle .
\end{aligned}
$$

As shown in [27, Lemma 3] and [28, Lemma 10], the condition in the above item (i) is automatically satisfied if $\boldsymbol{\Phi}, \boldsymbol{\Psi}, \tilde{\boldsymbol{\Phi}}, \tilde{\boldsymbol{\Psi}}$ are subsets of $L_{2}^{l o c}\left(\mathbb{R}^{d}\right)$ and $r, s$ are finite integers. The condition in the above item (ii) is simply the perfect reconstruction property in the test function space $\mathscr{D}\left(\mathbb{R}^{d}\right)$ for a pair of frequency-based affine systems.

Let $J$ be an integer and M be a $d \times d$ invertible real-valued matrix. Let $\Phi$ and $\Psi$ be subsets of $L_{1}^{\text {loc }}\left(\mathbb{R}^{d}\right)$. A nonhomogeneous $\mathrm{M}$-affine system is defined to be

$$
\operatorname{AS}_{J}(\Phi ; \Psi)=\left\{\phi_{\mathrm{M}^{J} ; \mathrm{k}}: \mathrm{k} \in \mathbb{Z}^{d}, \phi \in \Phi\right\} \cup \cup_{j=J}^{\infty}\left\{\psi_{\mathrm{M}^{j} ; \mathrm{k}}: \mathrm{k} \in \mathbb{Z}^{d}, \psi \in \Psi\right\} .
$$

To emphasize the role played by $\mathrm{M}$, we also use $\mathrm{AS}_{J}^{\mathrm{M}}(\Phi ; \Psi)$ instead of $\mathrm{AS}_{J}(\Phi ; \Psi)$. If $\Phi$, $\Psi$ are subsets of $L_{2}\left(\mathbb{R}^{d}\right)$, then we can define $\boldsymbol{\Phi}:=\{\widehat{\phi}: \phi \in \Phi\}$ and $\boldsymbol{\Psi}:=\{\widehat{\psi}: \psi \in \Psi\}$, where the Fourier transform used 
in this paper is defined to be $\widehat{f}(\xi):=\int_{\mathbb{R}^{d}} f(x) e^{-i x \cdot \xi} d x, \xi \in \mathbb{R}^{d}$ for $f \in L_{1}\left(\mathbb{R}^{d}\right)$. Then it is easy to see that the image of $\operatorname{AS}_{J}^{\mathrm{M}}(\Phi ; \Psi)$ under the Fourier transform is exactly $\operatorname{FAS}_{J}^{\mathrm{N}}(\boldsymbol{\Phi}, \boldsymbol{\Psi})$ with $\mathrm{N}=\left(\mathrm{M}^{\top}\right)^{-1}$. Moreover, the homogeneous M-affine system $\mathrm{AS}(\Psi)$ in (1.2) is simply the limiting system of $\operatorname{AS}_{J}(\Phi, \Psi)$ as $J \rightarrow-\infty$. See $[27,28]$ for more detail.

Frequency-based dual framelets can be completely characterized as follows:

Theorem 6.1. ([28, Theorem 11] and [27, Theorem 6]) Let $\mathrm{M}$ be a $d \times d$ real-valued invertible matrix such that $\mathrm{M}$ is expansive. Define $\mathrm{N}:=\left(\mathrm{M}^{\top}\right)^{-1}$. Let $\boldsymbol{\Phi}, \tilde{\boldsymbol{\Phi}}, \boldsymbol{\Psi}, \tilde{\boldsymbol{\Psi}}$ in $(6.2)$ be finite subsets of $L_{2}^{\text {loc }}\left(\mathbb{R}^{d}\right)$. Then the following statements are equivalent:

(i) $\left(\mathrm{FAS}_{J}(\boldsymbol{\Phi} ; \boldsymbol{\Psi}), \mathrm{FAS}_{J}(\tilde{\boldsymbol{\Phi}} ; \tilde{\boldsymbol{\Psi}})\right)$ is a frequency-based dual $\mathrm{M}$-framelet.

(ii) The following identities hold: for all $\mathbf{f}, \mathbf{g} \in \mathscr{D}\left(\mathbb{R}^{d}\right)$,

$$
\lim _{j \rightarrow+\infty} \sum_{\ell=1}^{r} \sum_{\mathbf{k} \in \mathbb{Z}^{d}}\left\langle\mathbf{f}, \boldsymbol{\varphi}_{\mathrm{N}^{j} ; \mathbf{0}, \mathrm{k}}^{\ell}\right\rangle\left\langle\tilde{\boldsymbol{\varphi}}_{\mathrm{N}^{j} ; \mathbf{0}, \mathrm{k}}^{\ell}, \mathbf{g}\right\rangle=(2 \pi)^{d}\langle\mathbf{f}, \mathbf{g}\rangle
$$

and

$$
\begin{aligned}
\sum_{\ell=1}^{r} \sum_{\mathrm{k} \in \mathbb{Z}^{d}}\left\langle\mathbf{f}, \boldsymbol{\varphi}_{I_{d} ; \mathbf{0}, \mathrm{k}}^{\ell}\right\rangle\left\langle\tilde{\boldsymbol{\varphi}}_{I_{d} ; \mathbf{0}, \mathrm{k}}^{\ell}, \mathbf{g}\right\rangle & +\sum_{\ell=1}^{s} \sum_{\mathrm{k} \in \mathbb{Z}^{d}}\left\langle\mathbf{f}, \boldsymbol{\psi}_{I_{d} ; \mathbf{0}, \mathrm{k}}^{\ell},\right\rangle\left\langle\tilde{\boldsymbol{\psi}}_{I_{d} ; \mathbf{0}, \mathrm{k}}^{\ell}, \mathbf{g}\right\rangle \\
& =\sum_{\ell=1}^{r} \sum_{\mathrm{k} \in \mathbb{Z}^{d}}\left\langle\mathbf{f}, \boldsymbol{\varphi}_{\mathrm{N} ; \mathbf{0}, \mathrm{k}}^{\ell}\right\rangle\left\langle\tilde{\boldsymbol{\varphi}}_{\mathrm{N} ; \mathbf{0}, \mathrm{k}}^{\ell}, \mathbf{g}\right\rangle .
\end{aligned}
$$

(iii) The following relations are satisfied:

$$
\lim _{j \rightarrow+\infty}\left\langle\sum_{\ell=1}^{r} \overline{\varphi^{\ell}\left(\mathrm{N}^{j} \cdot\right)} \tilde{\varphi}^{\ell}\left(\mathrm{N}^{j} \cdot\right), \mathbf{h}\right\rangle=\langle 1, \mathbf{h}\rangle, \quad \forall \mathbf{h} \in \mathscr{D}\left(\mathbb{R}^{d}\right)
$$

and

$$
\mathcal{I}_{\boldsymbol{\Phi}}^{\mathrm{k}}(\xi)+\mathcal{I}_{\boldsymbol{\Psi}}^{\mathrm{k}}(\xi)=\mathcal{I}_{\boldsymbol{\Phi}}^{\mathrm{Nk}}(\mathrm{N} \xi), \quad \text { a.e. } \xi \in \mathbb{R}^{d}, \mathrm{k} \in \mathbb{Z}^{d} \cup\left[\mathrm{N}^{-1} \mathbb{Z}^{d}\right],
$$

where $\mathcal{I}_{\boldsymbol{\Phi}}^{\mathrm{k}}(\xi):=\sum_{\ell=1}^{r} \overline{\varphi^{\ell}(\xi)} \tilde{\varphi}^{\ell}(\xi+2 \pi \mathrm{k}), \mathrm{k} \in \mathbb{Z}^{d}$ and $\mathcal{I}_{\boldsymbol{\Phi}}^{\mathrm{k}}:=0$ for $\mathrm{k} \in \mathbb{R}^{d} \backslash \mathbb{Z}^{d}$, and

$$
\mathcal{I}_{\Psi}^{\mathrm{k}}(\xi):=\sum_{\ell=1}^{s} \overline{\psi^{\ell}(\xi)} \tilde{\boldsymbol{\psi}}^{\ell}(\xi+2 \pi \mathrm{k}), \mathrm{k} \in \mathbb{Z}^{d} \quad \text { and } \quad \mathcal{I}_{\Psi}^{\mathrm{k}}:=0, \mathrm{k} \in \mathbb{R}^{d} \backslash \mathbb{Z}^{d} .
$$

Note that if item (i) holds for some $J \in \mathbb{Z}$, then it holds for all $J \in \mathbb{Z}$. Item (ii) follows from item (i) by considering the difference between two consecutive levels. (ii) $\Longrightarrow$ (i) can be proved by properly scaling (6.8) to every scale level $J$ and then by summing them up. The equivalence between items (ii) and (iii) follows from the simple relation (see [28, Lemma 10]):

$$
\sum_{\mathrm{k} \in \mathbb{Z}^{d}}\left\langle\mathbf{f}, \boldsymbol{\psi}_{U ; \mathbf{0}, \mathrm{k}}\right\rangle\left\langle\tilde{\boldsymbol{\psi}}_{U ; \mathbf{0}, \mathrm{k}}, \mathbf{g}\right\rangle=(2 \pi)^{d} \int_{\mathbb{R}^{d}} \sum_{\mathbf{k} \in \mathbb{Z}^{d}} \mathbf{f}(\xi) \overline{\mathbf{g}\left(\xi+2 \pi U^{-1} \mathrm{k}\right)} \overline{\boldsymbol{\psi}(U \xi)} \tilde{\boldsymbol{\psi}}(U \xi+2 \pi \mathrm{k}) d \xi
$$

with the series on the left converging absolutely and $U$ being a $d \times d$ invertible real-valued matrix.

According to the following result, a dual framelet filter bank is naturally linked to a frequency-based dual framelet.

Theorem 6.2. Let $\mathrm{M}$ be a $d \times d$ expansive integer matrix. Let $\Theta, a, b_{1}, \ldots, b_{s}, \tilde{a}, \tilde{b}_{1}, \ldots, \tilde{b}_{s} \in l_{0}\left(\mathbb{Z}^{d}\right)$ such that $\widehat{a}(\mathbf{0})=\widehat{\tilde{a}}(\mathbf{0})=1$. Define $\mathrm{N}:=\left(\mathrm{M}^{\top}\right)^{-1}, \varphi(\xi):=\prod_{j=1}^{\infty} \widehat{a}\left(\mathrm{~N}^{j} \xi\right)$ and $\tilde{\varphi}(\xi):=\prod_{j=1}^{\infty} \widehat{\tilde{a}}\left(\mathrm{~N}^{j} \xi\right)$ 
for $\xi \in \mathbb{R}^{d}$. Then $\varphi$ and $\tilde{\varphi}$ are well-defined functions in $L_{2}^{\text {loc }}\left(\mathbb{R}^{d}\right)$ satisfying $\varphi\left(\mathrm{M}^{\top} \xi\right)=\widehat{a}(\xi) \varphi(\xi)$ and $\tilde{\varphi}\left(\mathrm{M}^{\top} \xi\right)=\widehat{\tilde{a}}(\xi) \tilde{\varphi}(\xi)$ for all $\xi \in \mathbb{R}^{d}$. For $\ell=1, \ldots, s$, define

$$
\boldsymbol{\eta}(\xi):=\boldsymbol{\varphi}(\xi), \quad \tilde{\boldsymbol{\eta}}(\xi):=\widehat{\Theta}(\xi) \tilde{\boldsymbol{\varphi}}(\xi), \quad \boldsymbol{\psi}^{\ell}\left(\mathrm{M}^{\top} \xi\right):=\widehat{b}_{\ell}(\xi) \boldsymbol{\varphi}(\xi), \quad \text { and } \quad \tilde{\boldsymbol{\psi}}^{\ell}\left(\mathrm{M}^{\top} \xi\right):=\widehat{\tilde{b}_{\ell}}(\xi) \tilde{\boldsymbol{\varphi}}(\xi)
$$

Then $\left(\mathrm{FAS}_{J}\left(\{\boldsymbol{\eta}\} ;\left\{\boldsymbol{\psi}^{1}, \ldots, \boldsymbol{\psi}^{s}\right\}\right), \mathrm{FAS}_{J}\left(\{\tilde{\boldsymbol{\eta}}\} ;\left\{\tilde{\boldsymbol{\psi}}^{1}, \ldots, \tilde{\boldsymbol{\psi}}^{s}\right\}\right)\right)$ is a frequency-based dual $\mathrm{M}$-framelet for every (or some) $J \in \mathbb{Z}$ if and only if $\widehat{\Theta}(0)=1$ and $\left(\left\{\tilde{a} ; \tilde{b}_{1}, \ldots, \tilde{b}_{s}\right\},\left\{a ; b_{1}, \ldots, b_{s}\right\}\right)_{\Theta}$ is an OEP-based dual Mframelet filter bank, that is, for all $\omega \in \Omega_{\mathrm{M}}$ and $\xi \in \mathbb{R}^{d}$,

$$
\widehat{\Theta}\left(\mathrm{M}^{\top} \xi\right) \widehat{\tilde{a}}(\xi) \overline{\widehat{a}(\xi+2 \pi \omega)}+\widehat{\tilde{b}_{1}}(\xi) \overline{\widehat{b_{1}}(\xi+2 \pi \omega)}+\cdots+\widehat{\tilde{b}_{s}}(\xi) \overline{\widehat{b_{s}}(\xi+2 \pi \omega)}=\delta(\omega) \widehat{\Theta}(\xi) .
$$

One can prove Theorem 6.2 by directly verifying the conditions in Theorem 6.1. Framelets in $L_{2}\left(\mathbb{R}^{d}\right)$ using OEP-based dual M-framelet filter banks have been introduced in $[4,10]$ (also see [9]). A classical dual M-framelet filter bank discussed in previous sections is a particular case of OEPbased dual $\mathrm{M}$-framelet filter banks by taking $\Theta=\delta$. All our discussions in Sections 2, 3, and 4 can be carried over to OEP-based dual M-framelet filter banks. Under the condition $\widehat{a}(0)=\widehat{\tilde{a}}(0)=$ 1 and $\Theta=\delta$, Theorems 6.1 and 6.2 together show that the perfect reconstruction property of $\left(\left\{\tilde{a} ; \tilde{b}_{1}, \ldots, \tilde{b}_{s}\right\},\left\{a ; b_{1}, \ldots, b_{s}\right\}\right)$ is equivalent to the perfect reconstruction property of a frequency-based dual framelet $\left(\operatorname{FAS}_{0}\left(\{\tilde{\boldsymbol{\varphi}}\} ;\left\{\tilde{\boldsymbol{\psi}}^{1}, \ldots, \tilde{\boldsymbol{\psi}}^{s}\right\}\right), \operatorname{FAS}_{0}\left(\{\boldsymbol{\varphi}\} ;\left\{\boldsymbol{\psi}^{1}, \ldots, \boldsymbol{\psi}^{s}\right\}\right)\right)$.

Another application of Theorem 6.1 is the following result on tight framelets in $L_{2}\left(\mathbb{R}^{d}\right)$.

Proposition 6.3. ([28, Corollary 17]) Let $\mathrm{M}$ be a $d \times d$ expansive real-valued matrix and define $\mathrm{N}:=$ $\left(\mathrm{M}^{\top}\right)^{-1}$. Let $\boldsymbol{\Phi}$ and $\boldsymbol{\Psi}$ be finite subsets of $L_{2}^{\text {loc }}\left(\mathbb{R}^{d}\right)$. Then the following are equivalent:

(1) $\mathrm{FAS}_{J}(\boldsymbol{\Phi} ; \boldsymbol{\Psi})$ is a tight frame for $L_{2}\left(\mathbb{R}^{d}\right)$, that is, $\boldsymbol{\Phi}, \boldsymbol{\Psi} \subseteq L_{2}\left(\mathbb{R}^{d}\right)$, and for all $\mathbf{f} \in L_{2}\left(\mathbb{R}^{d}\right)$,

$$
\sum_{\boldsymbol{\varphi} \in \boldsymbol{\Phi}} \sum_{\mathbf{k} \in \mathbb{Z}^{d}}\left|\left\langle\mathbf{f}, \boldsymbol{\varphi}_{\mathrm{N}^{j} ; \mathbf{0}, \mathrm{k}}\right\rangle\right|^{2}+\sum_{j=J}^{\infty} \sum_{\boldsymbol{\psi} \in \boldsymbol{\Psi}} \sum_{\mathbf{k} \in \mathbb{Z}^{d}}\left|\left\langle\mathbf{f}, \boldsymbol{\psi}_{\mathrm{N}^{j} ; \mathbf{0}, \mathrm{k}}\right\rangle\right|^{2}=(2 \pi)^{d}\|\mathbf{f}\|_{L_{2}\left(\mathbb{R}^{d}\right)}^{2} .
$$

(2) $\left(\mathrm{FAS}_{J}(\boldsymbol{\Phi} ; \boldsymbol{\Psi}), \mathrm{FAS}_{J}(\boldsymbol{\Phi} ; \boldsymbol{\Psi})\right)$ is a frequency-based dual $\mathrm{M}$-framelet.

(3) $\lim _{j \rightarrow+\infty} \sum_{\boldsymbol{\varphi} \in \boldsymbol{\Phi}}\left\langle\left|\boldsymbol{\varphi}\left(\mathrm{N}^{j} \cdot\right)\right|^{2}, \mathbf{h}\right\rangle=\langle 1, \mathbf{h}\rangle$ for all $\mathbf{h} \in \mathscr{D}\left(\mathbb{R}^{d}\right)$ and for almost every $\xi \in \mathbb{R}^{d}$,

$$
\begin{aligned}
& \sum_{\boldsymbol{\varphi} \in \boldsymbol{\Phi}} \overline{\boldsymbol{\varphi}(\xi)} \boldsymbol{\varphi}(\xi+2 \pi \mathrm{k})+\sum_{\boldsymbol{\psi} \in \boldsymbol{\Psi}} \overline{\boldsymbol{\psi ( \xi )}} \boldsymbol{\psi}(\xi+2 \pi \mathrm{k}) \\
& =\sum_{\boldsymbol{\varphi} \in \boldsymbol{\Phi}} \overline{\boldsymbol{\varphi}(\mathrm{N} \xi)} \boldsymbol{\varphi}(\mathrm{N}(\xi+2 \pi \mathrm{k})), \quad \mathrm{k} \in \mathbb{Z}^{d} \cap\left[\mathrm{N}^{-1} \mathbb{Z}^{d}\right], \\
& \sum_{\boldsymbol{\varphi} \in \boldsymbol{\Phi}} \overline{\boldsymbol{\varphi}(\xi)} \boldsymbol{\varphi}(\xi+2 \pi \mathrm{k})+\sum_{\boldsymbol{\psi} \in \boldsymbol{\Psi}} \overline{\boldsymbol{\psi}(\xi)} \boldsymbol{\psi}(\xi+2 \pi \mathrm{k})=0, \mathrm{k} \in \mathbb{Z}^{d} \backslash\left[\mathrm{N}^{-1} \mathbb{Z}^{d}\right], \\
& \sum_{\boldsymbol{\varphi} \in \boldsymbol{\Phi}} \overline{\boldsymbol{\varphi}(\mathrm{N} \xi)} \boldsymbol{\varphi}(\mathrm{N}(\xi+2 \pi \mathrm{k}))=0, \quad \mathrm{k} \in\left[\mathrm{N}^{-1} \mathbb{Z}^{d}\right] \backslash \mathbb{Z}^{d} .
\end{aligned}
$$

(4) There exist subsets $\Phi, \Psi$ of $L_{2}\left(\mathbb{R}^{d}\right)$ such that $\{\widehat{\phi}: \phi \in \Phi\}=\boldsymbol{\Phi},\{\widehat{\psi}: \psi \in \Psi\}=\boldsymbol{\Psi}$, and $\operatorname{AS}_{J}(\Phi ; \Psi)$ is a tight frame for $L_{2}\left(\mathbb{R}^{d}\right)$ :

$$
\sum_{\phi \in \Phi} \sum_{\mathrm{k} \in \mathbb{Z}^{d}}\left|\left\langle f, \phi_{\mathrm{M}^{J} ; \mathrm{k}}\right\rangle\right|^{2}+\sum_{j=J}^{\infty} \sum_{\psi \in \Psi} \sum_{\mathrm{k} \in \mathbb{Z}^{d}}\left|\left\langle f, \psi_{\mathrm{M}^{j} ; \mathrm{k}}\right\rangle\right|^{2}=\|f\|_{L_{2}\left(\mathbb{R}^{d}\right)}^{2}, \quad \forall f \in L_{2}\left(\mathbb{R}^{d}\right) .
$$

Moreover, if any of (1)-(4) holds, then $\mathrm{AS}(\Psi)$ is a tight frame for $L_{2}\left(\mathbb{R}^{d}\right)$, that is, (1.3) holds.

We complete this section by discussing the issue of sparsity. Let $\widehat{\tilde{\phi}}:=\tilde{\varphi}, \widehat{\tilde{\psi}}:=\tilde{\psi}^{\ell}$ and $\tilde{b}:=\tilde{b}_{\ell}$ in Theorem 6.2. Assume that $\tilde{\psi} \in L_{2}\left(\mathbb{R}^{d}\right)$. Suppose that the filter $\tilde{b}$ has order $m$ vanishing moments. Let 
$\mathrm{k} \in \mathbb{Z}^{d}$. If an input data $v$ agrees with some polynomial of degree less than $m$ on the support of $\tilde{b}(\cdot-\mathrm{Mk})$, then it follows from Theorem 3.2 that the framelet coefficient $\left[\mathcal{T}_{\tilde{b}, \mathrm{M}} v\right](\mathrm{k})=0$. Therefore, if an input data $v$ can be well approximated by some polynomial of degree less than $m$ on the support of $\tilde{b}(\cdot-\mathrm{Mk})$, then the framelet coefficient $\left[\mathcal{T}_{\tilde{b}, \mathrm{M}} v\right](\mathrm{k})$ is negligible.

Now we look at the function setting. Since $M$ is expansive, a smooth function $f$ can be well approximated by its Taylor polynomial $T_{m-1}$ of degree less than $m$ near a point $\mathbf{M}^{-j} \mathbf{k} \approx x_{0}$. Since $\tilde{b}$ has order $m$ vanishing moments, by $\widehat{\tilde{\psi}}\left(\mathrm{M}^{\top} \xi\right)=\widehat{\tilde{b}}(\xi) \widetilde{\tilde{\phi}}(\xi)$, we see that $\tilde{\psi}$ also has order $m$ vanishing moments: $\langle\mathrm{p}, \tilde{\psi}\rangle=0$ for all $\mathrm{p} \in \Pi_{m-1}$. Consequently, if $\mathrm{M}$ is isotropic (that is, all the eigenvalues of $\mathrm{M}$ have the same modulus and $\mathrm{M}$ is similar to a diagonal matrix), then (see [8] for dimension one)

$$
\left|\left\langle f, \tilde{\psi}_{\mathrm{M}^{j} ; \mathrm{k}}\right\rangle\right|=\left|\left\langle f-T_{m-1}, \tilde{\psi}_{\mathrm{M}^{j} ; \mathrm{k}}\right\rangle\right| \leqslant C|\operatorname{det}(\mathrm{M})|^{-(m / d+1 / 2) j}, \quad j \rightarrow \infty,
$$

where the constant $C$ only depends on $\tilde{\psi}$ and $f$. Therefore, for a smooth function $f$, the framelet coefficients $\left\langle f, \psi_{\mathrm{M}^{j}: \mathrm{k}}\right\rangle$ decays to zero rapidly as the scale level $j \rightarrow+\infty$. The fast decay of framelet coefficents in (6.15) plays a critical role in establishing the sparse representation of wavelet or waveletlike expansions in $L_{2}\left(\mathbb{R}^{d}\right)$ (see $[1,2,8,13,14,35,36]$ ).

Moreover, it follows trivially from the perfect reconstruction condition in (4.5) that

$$
\min \left(\operatorname{vm}\left(b_{1}\right), \ldots, \operatorname{vm}\left(b_{s}\right)\right) \leqslant \operatorname{sr}(\tilde{a}), \quad \min \left(\operatorname{vm}\left(\tilde{b}_{1}\right), \ldots, \operatorname{vm}\left(\tilde{b}_{s}\right)\right) \leqslant \operatorname{sr}(a)
$$

with the equalities being true when $s=|\operatorname{det}(\mathrm{M})|-1$ (that is, the case of biorthogonal wavelet filter banks). Therefore, for the case of wavelets, the sparsity property of a discrete framelet transform is closely related to the sum rules of the filters $a$ and $\tilde{a}$ which in turn are closely related to the approximation properties of the shift-invariant spaces generated by the refinable functions $\phi$ and $\tilde{\phi}$. In other words, the orders of vanishing moments, which are crucial for sparsity of wavelets, are completely determined by the approximation properties of refinable functions, while this claim may no longer be true in general for framelets.

\section{Directional Tight Framelets in $L_{2}\left(\mathbb{R}^{d}\right)$}

In this section, we discuss an example of directional tight framelets in $L_{2}\left(\mathbb{R}^{d}\right)$. As argued in $[1,13,14]$ and many references therein, tensor product real-valued wavelets in $L_{2}\left(\mathbb{R}^{d}\right)$ are isotropic and can only handle the horizontal and vertical directions well in dimension two. Therefore, tensor product real-valued wavelets are often not efficient enough to capture directional singularities in high-dimensional signals such as images. Therefore, directional affine systems are of interest for high dimensional data analysis such as image processing.

Applying Proposition 6.3, we can easily obtain directional tight framelets in $L_{2}\left(\mathbb{R}^{d}\right)$ (see $[28$, Section 4$]$ for detail). Here we provide an example of directional tight framelets with finitely many directions which are tensor product of complex-valued tight framelets in dimension one. We start with a real-valued $C^{\infty}$ function $\boldsymbol{\theta}: \mathbb{R} \rightarrow \mathbb{R}$ such that $(\boldsymbol{\theta}(\xi))^{2}+(\boldsymbol{\theta}(1-\xi))^{2}=1$ for all $\xi \in \mathbb{R}, \boldsymbol{\theta}(\xi)=0$ for all $\xi<0$, and $\boldsymbol{\theta}(\xi)=1$ for all $\xi \geqslant 1$. Such functions $\boldsymbol{\theta}$ can be easily constructed, for example, see [8, Section 3.3.5] and [15, Lemma 4.2]. For $0<\varepsilon_{1}<\varepsilon_{2}<\varepsilon_{3}<\varepsilon_{4}$, we define $2 \pi$-periodic $C^{\infty}$ functions $\mathbf{a}_{\varepsilon_{1}, \varepsilon_{2}}$ and $\mathbf{b}_{\varepsilon_{1}, \varepsilon_{2}, \varepsilon_{3}, \varepsilon_{4}}$ by

$$
\mathbf{a}_{\varepsilon_{1}, \varepsilon_{2}}(\xi):= \begin{cases}1, & \text { if } \xi \in\left[-\varepsilon_{1}, \varepsilon_{1}\right], \\ 0, & \text { if } \xi \in\left[-\pi,-\varepsilon_{2}\right] \cup\left[\varepsilon_{2}, \pi\right), \\ \boldsymbol{\theta}\left(\frac{\varepsilon_{2}+\xi}{\varepsilon_{2}-\varepsilon_{1}}\right), & \text { if } \xi \in\left(-\varepsilon_{2},-\varepsilon_{1}\right), \\ \boldsymbol{\theta}\left(\frac{\varepsilon_{2}-\xi}{\varepsilon_{2}-\varepsilon_{1}}\right), & \text { if } \xi \in\left(\varepsilon_{1}, \varepsilon_{2}\right)\end{cases}
$$

and

$$
\mathbf{b}_{\varepsilon_{1}, \varepsilon_{2}, \varepsilon_{3}, \varepsilon_{4}}(\xi):= \begin{cases}1, & \text { if } \xi \in\left[\varepsilon_{2}, \varepsilon_{3}\right], \\ 0, & \text { if } \xi \in\left[0, \varepsilon_{1}\right] \cup\left[\varepsilon_{4}, 2 \pi\right), \\ \boldsymbol{\theta}\left(\frac{\xi-\varepsilon_{1}}{\varepsilon_{2}-\varepsilon_{1}}\right), & \text { if } \xi \in\left(\varepsilon_{1}, \varepsilon_{2}\right), \\ \boldsymbol{\theta}\left(\frac{\varepsilon_{4}-\xi}{\varepsilon_{4}-\varepsilon_{3}}\right), & \text { if } \xi \in\left(\varepsilon_{3}, \varepsilon_{4}\right) .\end{cases}
$$


When $\varepsilon_{2} \leqslant \pi, \mathbf{a}_{\varepsilon_{1}, \varepsilon_{2}}$ is a well-defined $2 \pi$-periodic $C^{\infty}$ function. When $\varepsilon_{4} \leqslant 2 \pi+\varepsilon_{1}, \mathbf{b}_{\varepsilon_{1}, \varepsilon_{2}, \varepsilon_{3}, \varepsilon_{4}}$ is a well-defined $2 \pi$-periodic $C^{\infty}$ function. Define $2 \pi$-periodic functions by

$$
\widehat{a}(\xi):=\mathbf{a}_{\varepsilon_{1}, \varepsilon_{2}}(\xi), \quad \widehat{b^{p}}(\xi):=\mathbf{b}_{\varepsilon_{1}, \varepsilon_{2}, \varepsilon_{3}, 2 \pi-\varepsilon_{3}}(\xi), \quad \widehat{b^{n}}(\xi):=\mathbf{b}_{\varepsilon_{1}, \varepsilon_{2}, \varepsilon_{3}, 2 \pi-\varepsilon_{3}}(-\xi)
$$

and functions $\varphi, \psi^{p}, \psi^{n}$ on $\mathbb{R}$ by

$$
\boldsymbol{\varphi}(\xi):=\prod_{j=1}^{\infty} \widehat{a}\left(2^{-j} \xi\right), \quad \boldsymbol{\psi}^{p}(\xi):=\widehat{b^{p}}(\xi / 2) \varphi(\xi / 2), \quad \boldsymbol{\psi}^{n}(\xi):=\widehat{b^{n}}(\xi / 2) \boldsymbol{\varphi}(\xi / 2) .
$$

Moreover, since $\boldsymbol{\varphi}, \boldsymbol{\psi}^{p}, \boldsymbol{\psi}^{n} \in L_{2}(\mathbb{R})$, we can define functions $\phi, \psi^{p}, \psi^{n} \in L_{2}(\mathbb{R})$ by

$$
\widehat{\phi}(\xi):=\varphi(\xi), \quad \widehat{\psi^{p}}(\xi):=\boldsymbol{\psi}^{p}(\xi), \quad \widehat{\psi^{n}}(\xi):=\boldsymbol{\psi}^{n}(\xi) .
$$

Under the following conditions

$$
0 \leqslant \pi-\varepsilon_{3} \leqslant \varepsilon_{1}<\varepsilon_{2}<\pi / 2 \quad \text { and } \quad 2 \varepsilon_{2}<\varepsilon_{3}<\pi,
$$

we can easily check that $\left\{a ; b^{p}, b^{n}\right\}$ is a tight 2 -framelet filter bank such that $\widehat{\phi}$ is supported inside $\left[-2 \varepsilon_{2}, 2 \varepsilon_{2}\right], \widehat{\psi^{p}}$ is supported inside $\left[2 \varepsilon_{1}, 4 \varepsilon_{2}\right]$, and $\widehat{\psi^{n}}(\xi)=\widehat{\psi^{p}}(-\xi)$. Though $\phi$ is a real-valued function, the wavelet functions $\psi^{p}, \psi^{n}$ are complex-valued functions. Similarly, though the low-pass filter $a$ has real coefficients, the two high-pass filters $b^{p}, b^{n}$ have complex coefficients. By Theorem 6.2 and Proposition 6.3, we see that $\operatorname{FAS}_{J}\left(\{\boldsymbol{\varphi}\} ;\left\{\boldsymbol{\psi}^{p}, \boldsymbol{\psi}^{n}\right\}\right)$ is a frequency-based tight 2-framelet for $L_{2}(\mathbb{R})$. Moreover, both $\operatorname{AS}_{J}\left(\{\phi\} ;\left\{\psi^{p}, \psi^{n}\right\}\right)$ and $\operatorname{AS}\left(\left\{\psi^{p}, \psi^{n}\right\}\right)$ are tight 2-framelets for $L_{2}(\mathbb{R})$. See Figure 1 for the graphs of $\widehat{a}, \widehat{b^{p}}, \widehat{b^{n}}$ and $\phi, \psi^{p}$ with the choice $\varepsilon_{1}=0.3, \varepsilon_{2}=1.47$, and $\varepsilon_{3}=2.94$.

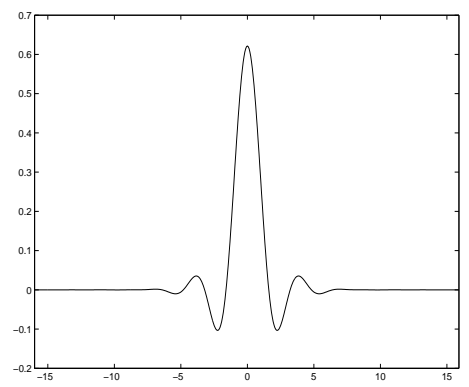

(a) $\phi$

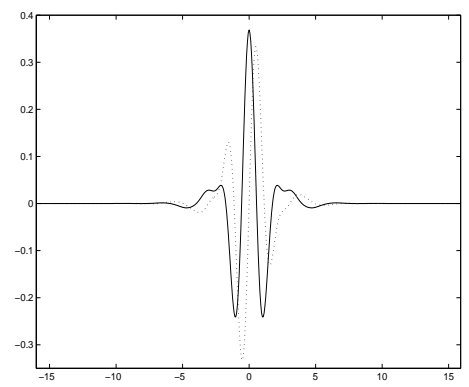

(b) $\psi^{p}$

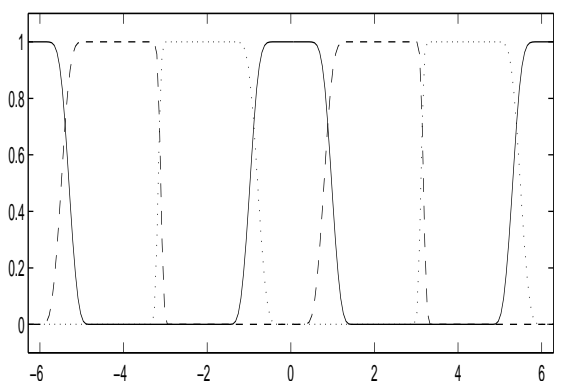

(c) $\widehat{a}, \widehat{b^{p}}, \widehat{b^{n}}$

Figure 1. (a) is the graph of the refinable function $\phi$ with the low-pass filter $a$. (b) is the graph of the wavelet function $\psi^{p}$ (solid line for the real part and dotted line for the imaginary part). (c) is the graph of $\widehat{a}$ (in solid line), $\widehat{b^{p}}$ (in dashed line), and $\widehat{b^{n}}$ (in dotted line).

We now consider the tensor product framelets in dimension two. Recall that the tensor product $u \otimes v$ of $u$ and $v$ simply means $u \otimes v\left(\xi_{1}, \xi_{2}\right):=u\left(\xi_{1}\right) v\left(\xi_{2}\right)$. In dimension two, we have the tensor product tight $2 I_{2}$-framelet filter bank:

$$
\left\{a \otimes a ; a \otimes b^{p}, a \otimes b^{n}, b^{p} \otimes a, b^{p} \otimes b^{p}, b^{p} \otimes b^{n}, b^{n} \otimes a, b^{n} \otimes b^{p}, b^{n} \otimes b^{n}\right\},
$$

which consists of one low-pass filter $a \otimes a$ having real coefficients and 8 high-pass filters having complex coefficients. Define $\mathrm{M}:=2 I_{2}$ and $\mathrm{N}:=2^{-1} I_{2}$. By Theorem 6.2 and Proposition 6.3,

$$
\operatorname{FAS}_{J}\left(\{\boldsymbol{\varphi} \otimes \boldsymbol{\varphi}\} ;\left\{\boldsymbol{\varphi} \otimes \boldsymbol{\psi}^{p}, \boldsymbol{\varphi} \otimes \boldsymbol{\psi}^{n}, \boldsymbol{\psi}^{p} \otimes \boldsymbol{\varphi}, \boldsymbol{\psi}^{p} \otimes \boldsymbol{\psi}^{p}, \boldsymbol{\psi}^{p} \otimes \boldsymbol{\psi}^{n}, \boldsymbol{\psi}^{n} \otimes \boldsymbol{\varphi}, \boldsymbol{\psi}^{n} \otimes \boldsymbol{\psi}^{p}, \boldsymbol{\psi}^{n} \otimes \boldsymbol{\psi}^{n}\right\}\right)
$$


is a frequency-based tight $2 I_{2}$-framelet for $L_{2}\left(\mathbb{R}^{2}\right)$ and both

$$
\mathrm{AS}_{J}\left(\{\phi \otimes \phi\},\left\{\phi \otimes \psi^{p}, \phi \otimes \psi^{n}, \psi^{p} \otimes \phi, \psi^{p} \otimes \psi^{p}, \psi^{p} \otimes \psi^{n}, \psi^{n} \otimes \phi, \psi^{n} \otimes \psi^{p}, \psi^{n} \otimes \psi^{n}\right\}\right)
$$

and $\operatorname{AS}\left(\left\{\phi \otimes \psi^{p}, \phi \otimes \psi^{n}, \psi^{p} \otimes \phi, \psi^{p} \otimes \psi^{p}, \psi^{p} \otimes \psi^{n}, \psi^{n} \otimes \phi, \psi^{n} \otimes \psi^{p}, \psi^{n} \otimes \psi^{n}\right\}\right)$ are tight frames for $L_{2}\left(\mathbb{R}^{2}\right)$. Splitting the real and imaginary parts of the filters in (7.6), we end up with a directional tight $2 I_{2}$-framelet filter bank and their associated functions are the real and imaginary parts of the generators in (7.7), whose graphs are given in Figure 2. From Figure 2, we can see that the tensor product tight framelets have four directions. Tight $2 I_{2}$-framelets with more directions can be constructed similarly using more high-pass filters instead of just two high-pass filters $b^{p}$ and $b^{n}$. For example, tight $2 I_{2}$-framelets with eight directions can be constructed using four high-pass filters $b^{p_{1}}, b^{p_{2}}$ and $b^{n_{1}}, b^{n_{2}}$.
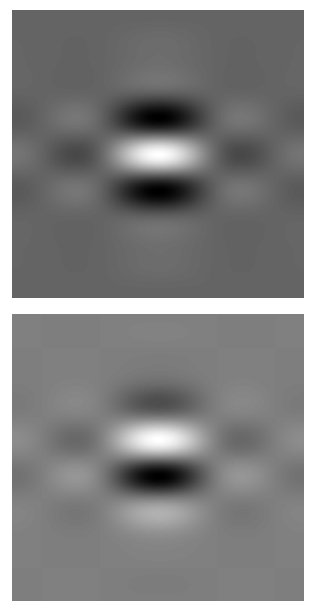
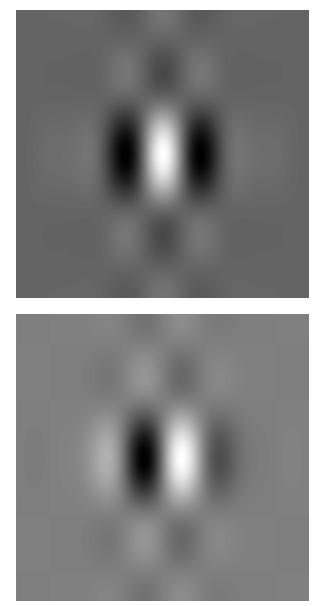
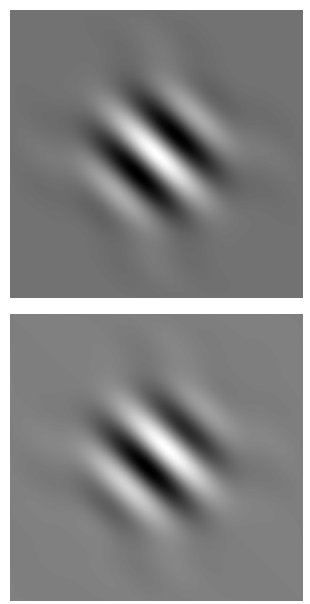
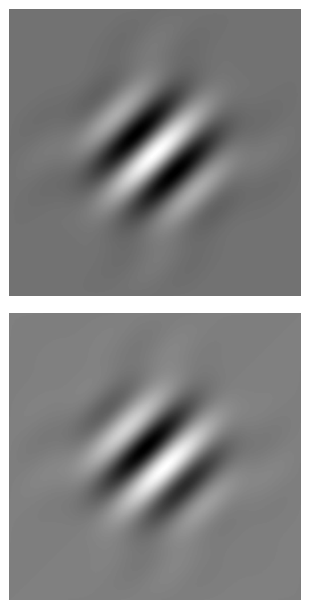

Figure 2. The first row is the real parts and the second row is the imaginary part of the tensor product complex wavelet functions. Both the real parts and the imaginary parts exhibit four directions.

We finish this paper by some remarks. First of all, the above constructed directional tight framelets are quite different from those systems developed in $[1,13,14,28]$. (Homogeneous) wavelets/framelets with composite dilations are proposed in [14] to remedy the lack of directionality of classic wavelets, while [28] employs nonstationary (nonhomogeneous) wavelet structure to achieve directionality by using the standard dilation matrix $2^{j} I_{2}$ at the scale level $j$. The affine systems developed in [1] for curvelets, in [13] for shearlets, and in [28] for directional tight framelets have more and more directions as the scale level increases and the elements of the generators are highly anisotropic by obeying the hyperbolic rule as discussed in [1]. The directionality in $[1,13,14]$ is achieved by taking advantages of different anisotropic dilation matrices at the scale level $j$, while the directionality in [28] is achieved by using different framelet generators directly through rotation, but still employing the isotropic dilation matrix $2^{j} I_{2}$ at the scale level $j$. The directional tight framelets constructed in [28] have associated underlying filter banks but the redundancy of the system in $[28]$ is much higher than those in $[1,13]$ which are optimal for carton-like images $([1,13])$. However, the tight framelets constructed above have only limited directions and are similar in some sense to the complex wavelets in [38]. Hence, their asymptotic approximation rates will be similar to those of classical tensor product real-valued wavelets. However, for a given digital image, its resolution is finite and therefore, it has only finitely many directions. Hence, the constructed tight framelets are of particular interest in their applications to digital image processing. 
Acknowledgements. This research is supported in part by NSERC Canada. The author thanks Zhenpeng Zhao at University of Alberta for plotting the graphs in Figures 1 and 2.

\section{References}

[1] E. J. Candès, D. L. Donoho. New tight frames of curvelets and optimal representations of objects with $C^{2}$ singularities, Comm. Pure Appl. Math. 56 (2004), 219-266.

[2] C. K. Chui. An introduction to wavelets. Academic Press, Inc., Boston, MA, 1992.

[3] C. K. Chui, W. He. Compactly supported tight frames associated with refinable functions. Appl. Comput. Harmon. Anal. 8 (2000), 293-319.

[4] C. K. Chui, W. He, J. Stöckler. Compactly supported tight and sibling frames with maximum vanishing moments, Appl. Comput. Harmon. Anal. 13 (2002), 224-262.

[5] A. Cohen, I. Daubechies. A stability criterion for biorthogonal wavelet bases and their related subband coding scheme. Duke Math. J. 68 (1992), 313-335.

[6] A. Cohen, I. Daubechies, J.-C. Feauveau. Biorthogonal bases of compactly supported wavelets. Comm. Pure Appl. Math. 45 (1992), 485-560.

[7] I. Daubechies. Orthonormal bases of compactly supported wavelets, Comm. Pure Appl. Math. 41 (1988), 909-996.

[8] I. Daubechies. Ten lectures on wavelets. SIAM, CBMS Series, 1992.

[9] I. Daubechies, B. Han. Pairs of dual wavelet frames from any two refinable functions, Constr. Approx. 20 (2004), 325-352.

[10] I. Daubechies, B. Han, A. Ron, Z. Shen. Framelets: MRA-based constructions of wavelet frames. Appl. Comput. Harmon. Anal. 14 (2003), 1-46.

[11] M. Ehler. On multivariate compactly supported bi-frames, J. Fourier Anal. Appl. 13 (2007), 511-532.

[12] M. Ehler, B. Han. Wavelet bi-frames with few generators from multivariate refinable functions, Appl. Computat. Harmon. Anal. 25 (2008), 407-414.

[13] K. Guo, D. Labate. Optimally sparse multidimensional representation using shearlets. SIAM J. Math. Anal. 39 (2007), $298-318$.

[14] K. Guo, D. Labate, W.-Q Lim, G. Weiss, E. Wilson. Wavelets with composite dilations and their MRA properties. Appl. Comput. Harmon. Anal. 20 (2006), 202-236.

[15] B. Han. On dual wavelet tight frames. Appl. Comput. Harmon. Anal. 4 (1997), 380-413.

[16] B. Han. Approximation properties and construction of Hermite interpolants and biorthogonal multiwavelets, J. Approx. Theory. 110 (2001), 18-53.

[17] B. Han. Symmetry property and construction of wavelets with a general dilation matrix, Linear Algebra and its Applications. 353 (2002), 207-225

[18] B. Han. Computing the smoothness exponent of a symmetric multivariate refinable function, SIAM J. Matrix Anal. Appl. 24 (2003), 693-714.

[19] B. Han. Vector cascade algorithms and refinable function vectors in Sobolev spaces, J. Approx. Theory. 124 (2003), $44-88$.

[20] B. Han. Compactly supported tight wavelet frames and orthonormal wavelets of exponential decay with a general dilation matrix, J. Comput. Appl. Math. 155 (2003), 43-67.

[21] B. Han. Classification and construction of bivariate subdivision schemes, Proceedings on Curves and Surfaces Fitting: Saint-Malo 2002, A. Cohen, J.-L. Merrien, and L. L. Schumaker eds., (2003), 187-197.

[22] B. Han. Symmetric multivariate orthogonal refinable functions, Appl. Comput. Harmon. Anal.. 17 (2004), 277-292.

[23] B. Han. Matrix extension with symmetry and applications to symmetric orthonormal complex M-wavelets. J. Fourier Anal. Appl. 15 (2009), 684-705.

[24] B. Han. Dual multiwavelet frames with high balancing order and compact fast frame transform. Appl. Comput. Harmon. Anal. 26 (2009), 14-42.

[25] B. Han. The structure of balanced multivariate biorthogonal multiwavelets and dual multiframelets. Math. Comp. 79 (2010), 917-951.

[26] B. Han. Symmetric orthonormal complex wavelets with masks of arbitrarily high linear-phase moments and sum rules. Adv. Comput. Math. 32 (2010), 209-237.

[27] B. Han. Pairs of frequency-based nonhomogeneous dual wavelet frames in the distribution space, Appl. Comput. Harmon. Anal. 29 (2010), 330-353.

[28] B. Han. Nonhomogeneous wavelet systems in high dimensions, Appl. Comput. Harmon. Anal. 32 (2012), $169-196$.

[29] B. Han, Q. Mo. Splitting a matrix of Laurent polynomials with symmetry and its application to symmetric framelet filter banks, SIAM J. Matrix Anal. Appl. 26 (2004), 97-124.

[30] B. Han, Q. Mo. Symmetric MRA tight wavelet frames with three generators and high vanishing moments, Appl. Comput. Harmon. Anal. 18 (2005), 67-93.

[31] B. Han, Z. Shen. Dual wavelet frames and Riesz bases in Sobolev spaces, Constr. Approx. 29 (2009), 369-406.

[32] B. Han, X. S. Zhuang. Analysis and construction of multivariate interpoalting refinable function vectors, Acta Appl. Math. 107 (2009), 143-171.

[33] R. Q. Jia. Approximation properties of multivariate wavelets, Math. Comp. 67 (1998), 647-665. 
[34] M. J. Lai, J. Stöckler. Construction of multivariate compactly supported tight wavelet frames, Appl. Comput. Harmon. Anal. 21 (2006), 324-348.

[35] S. Mallat. A wavelet tour of signal processing. Third edition. Elsevier/Academic Press, Amsterdam, 2009.

[36] Y. Meyer. Wavelets and operators. Cambridge University Press, Cambridge, 1992.

[37] A. Ron, Z. Shen. Affine systems in $L_{2}\left(\mathbb{R}^{d}\right)$ : the analysis of the analysis operator, J. Funct. Anal. 148 (1997), $408-447$.

[38] I. W. Selesnick, R. G. Baraniuk, N. G. Kingsbury. The dual-tree complex wavelet transform. IEEE Signal Proc. Magazine, 123 (2005), 123-151.

[39] G. Strang, T. Nguyen. Wavelets and filter banks. Wellesley College, 2nd edition, 1996.

[40] M. Vetterli, J. Kovacěvić. Wavelets and subband coding. Prentice Hall Signal Processing Series, 1995. 